Original Research Paper

\title{
Molecular Characterization and Differentiation of Proteases Isolated from Different Aspergillus Fungal Species
}

\author{
Samia Abd Allah Abdal-Aziz and Safaa M. Ali \\ Department of Nucleic Acid Research, Genetic Engineering and Biotechnology Research Institute, \\ City of Scientific Research and Technological Applications, New Borg El-Arab City 21934, Alexandria, Egypt
}

Article history

Received: 19-10-2020

Revised: 09-02-2021

Accepted: 10-02-2021

Corresponding Author:

Safaa M. Ali

Department of Nucleic Acid Research, Genetic Engineering and Biotechnology Research Institute, City of Scientific Research and Technological Applications, New Borg ElArab City 21934, Alexandria, Egypt

Email: Safaa.mohamedali@yahoo.com

\begin{abstract}
A comparative molecular study of Aperigiullus protease using sequence of protease gene representative 25 different Aspergillus species (Aspergillus fumigatus, Aspergillus oryzae, Aspergillus flavus, Aspergillus novofumigatus, Aspergillus viridinutans, Aspergillus pseudotamarii, Aspergillus clavatus, Aspergillus sojae, Aspergillus fischeri, Aspergillus caelatus, Aspergillus novofumigatus, Aspergillus campestris, Aspergillus costaricaensis, Aspergillus candidus, Aspergillus neoniger, Aspergillus mulundensis, Aspergillus ibericus, Aspergillus niger, Aspergillus piperis, Aspergillus eucalypticola, Aspergillus vadensis, Aspergillus steynii, and Aspergillus lentulus) have been described for several properties. To summarise the Asperigillus protease correlation, alignment of multiple sequence and phylogenetic tree assembly were carried out. 59 Protease gene amino acid sequence used for the constructed phylogenetic tree that showed the specific protease clusters of several species of Aspergillus (Aspergillus oryzae, Aspergillus fumigatus, Aspergillus clavatus, Aspergillus niger, Aspergillus nidulans, Aspergillus viridinutans, Aspergillus flavus and Aspergillus fischeri). Protease gene amino acid sequences acquired from NCBI. The homology sequence level was done using amino acid sequence. Among these proteases, the distributions of the commonly observed motifs were analysed. Protease protein sequences indicate that the structure and enzymatic function are involved.
\end{abstract}

Keywords: Protease, Aspergillus, Sequence, Amino Acid, Phylogenetic

\section{Introduction}

Proteases organise a wide and matrix cluster of digestive enzymes in the food, detergent and leather, medical, pharmaceutical and biotechnology industries with essential applications (Ramakrishna et al., 2010). Protease enzymes are multipurpose and represent an essential variety of enzymes for the variability of their biotechnological and physiological functions (Silva et al., 2011). Animals, plants and microorganisms, which constitute about $60 \%$ of the global market for enzymes, can produce those (Nirmal et al., 2011). Filamentous fungi were being used for the development of different structural proteins. In particular, a few species of Aspergillus have been misused as essential sources of extracellular protease counting chemicals (De Castro and Sato, 2014a; Li et al., 2014). Aspergillus produces aimed at classes for instance Aspergillus Sojae, Aspergillus niger and Aspergillus oryzae have approved their usage inside the food manufacturing assimilated a mostly documented as nontoxic grade as of the US medicine management and food (Heerd et al., 2012). It is possible to classify proteases as either acidic protease, impartial protease or soluble protease, corresponding to their ideal $\mathrm{pH}$ reactions. Proteases, especially impartial proteases, make up the world's largest amount of mechanical chemicals (Kasana et al., 2011). Since their specific benefits, neutral proteases are broadly linked within the nourishment (Tavano, 2013; Yuzuki et al., 2015), bolster (Zhang et al., 2014), pharmaceutical (Umeadi et al., 2008) and calfskin (Asker et al., 2013), counting a gentle catalysis preparation and low level of contamination and high abdication. Impartial protease is often used in the food industry besides debiting soy sauce (Machida et al., 2005) and brewing (Wang et al., 2013). The unbiased protease, however, is largely destitute in warm solidity and is easily deactivated, limiting its application in a few companies requiring high temperatures (Xiao-Lin et al., 2018). Much consideration has been given to the application of proteases to the hydrolysis of creature and plant proteins to extend their natural and useful properties. In a number of studies, antioxidant exercises 
of protein hydrolysates are broadly detailed (Zhou et al., 2012). Proteases hypothesized that the antioxidant characteristics of peptides come from their capacities to inactivate Responsive Oxygen Species (ROS), rummage free radicals, chelate prooxidative move metals and diminish hydroperoxides (De Castro and Sato, 2014b). Alkaline soluble proteases are particularly intrigued by their potential applications as a cleaning substance added within the cleanser industry (Anwar and Saleemuddin, 1998; Gupta et al., 2002). Proteases are clustered into four robotic classes: The cysteine, serine proteases, metallo and amino corrosive proteases. Possibly the best individuals were Chymotrypsin, Trypsin, Elastase and Subtilisin. Alkaline proteases were being clustered into 20 families, categorized into 6 clans with direct ancestors, depending on their own strong interaction (Barrett, 1994). The six particular catalytic proteases sorts that can be situated documented are the threonine, serine, aspartic, cysteine, metallo-proteases and glutamic. Similarities of amino acid sequences, three-dimensional constructions and proteolytic enzymes modes of action helped decipher their evolutionary course (Nishihira and Tachikawa, 1999; Morya et al., 2012). Protein sequences of developed enzymes have been described to be characterised in silico (Yadav et al., 2009; Dubey et al., 2010; Yadav et al., 2010). The characterization of Silico proteases produced by the Aspergillus species is reported in this study. Using bioinformatics tools, multiple sequence alignment, homology search, phylogenetic tree construction and superfamily distribution were analysed. Studies of the application of new sources of proteolysis enzymes are therefore critical for advancing knowledge about bioactive proteins.

\section{Materials and Methods}

\section{Isolation}

Sequences of protease genes were selected from NCBI as row data to differentiate among the different species of Asperigillus (Aspergillus oryzae, Aspergillus fumigatus, Aspergillus clavatus, Aspergillus pseudotamarii, Aspergillus novofumigatus, Aspergillus viridinutans, Aspergillus sojae, Aspergillus flavus, Aspergillus campestris, Aspergillus fischeri, Aspergillus novofumigatus, Aspergillus candidus, Aspergillus costaricaensis, Aspergillus mulundensis, Aspergillus neoniger, Aspergillus niger, Aspergillus ibericus, Aspergillus eucalypticola, Aspergillus piperis, Aspergillus vadensis, Aspergillus steynii, Aspergillus lentulus, Aspergillus fischeri and Aspergillus lentulus). On the Proteomic database financial links \{Clustal W, ProtParam, Protein calculator $\}$, list of FASTA format was used for subsequent research. To determine the molecular weights of the different proteases diverse programs were used for calculation.

\section{Detection of Restriction Site}

Different program were used to detect the site of restriction enzymes (clone manger, restriction mapper)

\section{Differentiation between Protease Genes}

Statistical analyses were used to determine the differentiation between the different sp. of fungal isolates.

\section{Results}

\section{Molecular Characterization of Asperigillus Protease using Nucleotide Sequences}

25 different species of Asperigillus \{Aspergillus oryzae (D00350.1, S79617.1, S75278.1, D10062.1, X54726.1 A. and X17561.1); Aspergillus fumigatus (AB807664.1, X66935.1, Z11580.1 and XM_746558.1); Aspergillus novofumigatus (XM_024823318.1); Aspergillus flavus (XM_002374250.1 and AF324246.1); Aspergillus clavatus (GQ925367.1 and XM_001272037.1); Aspergillus pseudotamarii (XM_032052352.1 and XM_032052352.1); Aspergillus viridinutans (AY590135.1 and AY590136.1); Aspergillus sojae (MG867728.1); Aspergillus fischeri (XM_001266851.1); Aspergillus caelatus (XM_032073130.1); Aspergillus novofumigatus (XM_024825725.1); Aspergillus candidus (XM_024813742.1); Aspergillus campestris (XM_024833434.1); Aspergillus piperis (XM_025662057.1); Aspergillus mulundensis (XM_026751684.1); Aspergillus costaricaensis (XM_025678012.1); Aspergillus niger (L19059.1); Aspergillus ibericus (XM_025719167.1); Aspergillus neoniger (XM_025625888.1); Aspergillus vadensis (XM_025711220.1); Aspergillus eucalypticola (XM_025536473.1); Aspergillus steynii (XM_024843524.1); Aspergillus fischeri (XM_001258144.1); Aspergillus lentulus (XM_033554602.1); Aspergillus lentulus (XM_033555156.1)\} were used. Sequences of proteases acquired from GenBank symbolise diverse species of Aspergillus were designated as multiple sequence alignment, homology quest, phylogenetic tree creation and superfamily quest with different bioinformatics tools. Data on gene bank reflect that Aspergillus oryzae was the most organism used for protease production.

\section{PCR-Restriction Fragment Length Polymorphism (PCR-RFLP)}

Protease gene analysis with the use of various restriction enzymes (StuI, AflII, Bsp1407I, EcoP15I, PasI, SpeI, Tsp45I, BfiI, KpnI, MmeI, HindII, AccI 33, AgsI, Hpy99I, TauI, BccI, CfrI, SapI, BsaBI, BtrI, MslI, PsiI, PvuII, StuI, ApoI, BclI, BglII, Bsp1407I, BspHI, EcoRI, Esp3I, HindIII, VspI, XbaI, BtsI, Eco57I, 
PflMI, SphI, AatII, AloI, ArsI, BsgI, HaeIV, PstI and SacII) were done. The digested products were fractionated in the different Aspergillus species (presented in Table 1). Analysis result of these genes from NCBI showed variation in restriction sites between different Aspergillus species. To preserve the functional activity of the protein that reflects the variation between the digested protease genes related to genes listed as presented in the supplementary file (Tables 1-36 supplementary) protease genes, maintaining the core structural component of the active site is crucial.

\section{Molecular Characterization of Asperigillus Protease using Amino Acid Sequences}

For comparative motif analysis, 59 of amino acid sequences of different types of Aspergillus species were used to detect the variation between the different sources and by it can detect the structure of protease isolated from different Aspergillus species. The amino acid sequences which were collected from NCBI were classified as follows: 20 Protease gene amino acid sequences isolated from Aspergillus fumigatus (XP_751651.1, CAA77666.1, A CAA75805.1, EDP50543.1, XP_750914.1, 1905286A1, B0Y708.1， EAL89613.1， XP_751651.1， CAA75806.1, Q4WH48, CAA75804.1, XP_749017.1, B0Y1V8.1, P28296.2, P41748.2, EAL86979.1, B0XRV0.1, O42630.1 P0C959.1), 14 Protease gene amino acid sequences isolated from Aspergillus oryzae (Q2U319, Q2UDE1, Q8NK92.2, Q2UUW3, AAC60533.1, Q8NKB2, Q8NKB6.1, AAB20819.1, Q06902.2, BAA00951.1, Q9Y8E3.2, P46076.2, Q2TWA0, Q8NKB5), 9 Protease gene amino acid sequences isolated from Aspergillus niger (A2R3L3.1, XP_001391470.1, P55325.1, P24665.1, A2Q7V4.1, CAK44878.1, Q00070, P55325.1, A2R2G1.1), 7 Protease gene amino acid sequences isolated from Aspergillus clavatus (A1CIA7.1, A1CBR4.1, ACX47962.1, A1CBR4.1, EAW10612.1, EAW10612.

Table 1: Characterization of protease genes extracted from Different Aspergillus spp. based on (no. of nucleotides, no. of amino acids obtained after translation

\begin{tabular}{|c|c|c|c|c|}
\hline Organisms & Accession no & No. of nucleotide & No of amino acid & $\begin{array}{l}\text { Expected molecular } \\
\text { weight of protein }(\mathrm{kDa})\end{array}$ \\
\hline Aspergillus lentulus & XM_033555156.1 & 1182 & 394 & 41.72 \\
\hline Aspergillus fischeri & XM_001258144.1 & 1935 & 645 & 68.29 \\
\hline Aspergillus lentulus & XM_033554602.1 & 1212 & 404 & 42.78 \\
\hline Aspergillus steynii & XM_024843524.1 & 1212 & 404 & 42.78 \\
\hline Aspergillus vadensis & XM_025711220.1 & 1254 & 418 & 44.26 \\
\hline Aspergillus piperis & XM_025662057.1 & 1682 & 561 & 59.37 \\
\hline Aspergillus eucalypticola & XM_025536473.1 & 1252 & 417 & 44.19 \\
\hline Aspergillus ibericus & XM_025719167.1 & 1447 & 482 & 51.07 \\
\hline Aspergillus niger & L19059.1 & 2993 & 998 & 105.64 \\
\hline Aspergillus neoniger & XM_025625888.1 & 1263 & 421 & 44.58 \\
\hline Aspergillus mulundensis & XM_026751684.1 & 1209 & 403 & 42.67 \\
\hline Aspergillus costaricaensis & XM_025678012.1 & 1251 & 417 & 44.15 \\
\hline Aspergillus candidus & XM_024813742.1 & 1560 & 520 & 55.06 \\
\hline Aspergillus novofumigatus & XM_024825725.1 & 1212 & 404 & 42.78 \\
\hline Aspergillus campestris & XM_024833434.1 & 1212 & 404 & 42.78 \\
\hline Aspergillus fischeri & XM_001266851.1 & 1212 & 404 & 42.78 \\
\hline Aspergillus caelatus & XM_032073130.1 & 1212 & 404 & 42.78 \\
\hline Aspergillus sojae & MG8̄67728.1 & 1212 & 404 & 42.78 \\
\hline Aspergillus viridinutans & AY590136.1 & 1192 & 397 & 42.07 \\
\hline Aspergillus viridinutans & AY590135.1 & 1192 & 397 & 42.07 \\
\hline Aspergillus pseudotamarii & XM_032052352.1 & 2375 & 792 & 83.82 \\
\hline Aspergillus clavatus & GQ925367.1 & 2375 & 792 & 83.82 \\
\hline Aspergillus clavatus & XM_001272037.1 & 1212 & 404 & 42.78 \\
\hline Aspergillus flavus & XM_002374250.1 & 1212 & 404 & 42.78 \\
\hline Aspergillus flavus & $\mathrm{AF} 324246.1$ & 1886 & 629 & 66.57 \\
\hline Aspergillus novofumigatus & XM_024823318.1 & 2315 & 772 & 81.71 \\
\hline Aspergillus fumigatus & XM_746558.1 & 1212 & 404 & 42.78 \\
\hline Aspergillus.fumigatus & $\mathrm{Z} 11 \overline{5} 80.1$ & 2163 & 721 & 76.34 \\
\hline Aspergillus.fumigatus & X66935.1 & 758 & 253 & 26.75 \\
\hline Aspergillus fumigatus & AB807664.1 & 714 & 238 & 25.20 \\
\hline Aspergillus oryzae & X17561.1 & 1468 & 489 & 51.81 \\
\hline Aspergillus oryzae & X54726.1 & 1820 & 607 & 64.24 \\
\hline Aspergillus oryzae & D10062.1 & 2601 & 867 & 91.80 \\
\hline Aspergillus oryzae & S75278.1 & 1820 & 607 & 64.24 \\
\hline Aspergillus oryzae & S79617.1 & 2600 & 867 & 91.77 \\
\hline Aspergillus oryzae & D00350.1 & 1182 & 394 & 41.72 \\
\hline
\end{tabular}




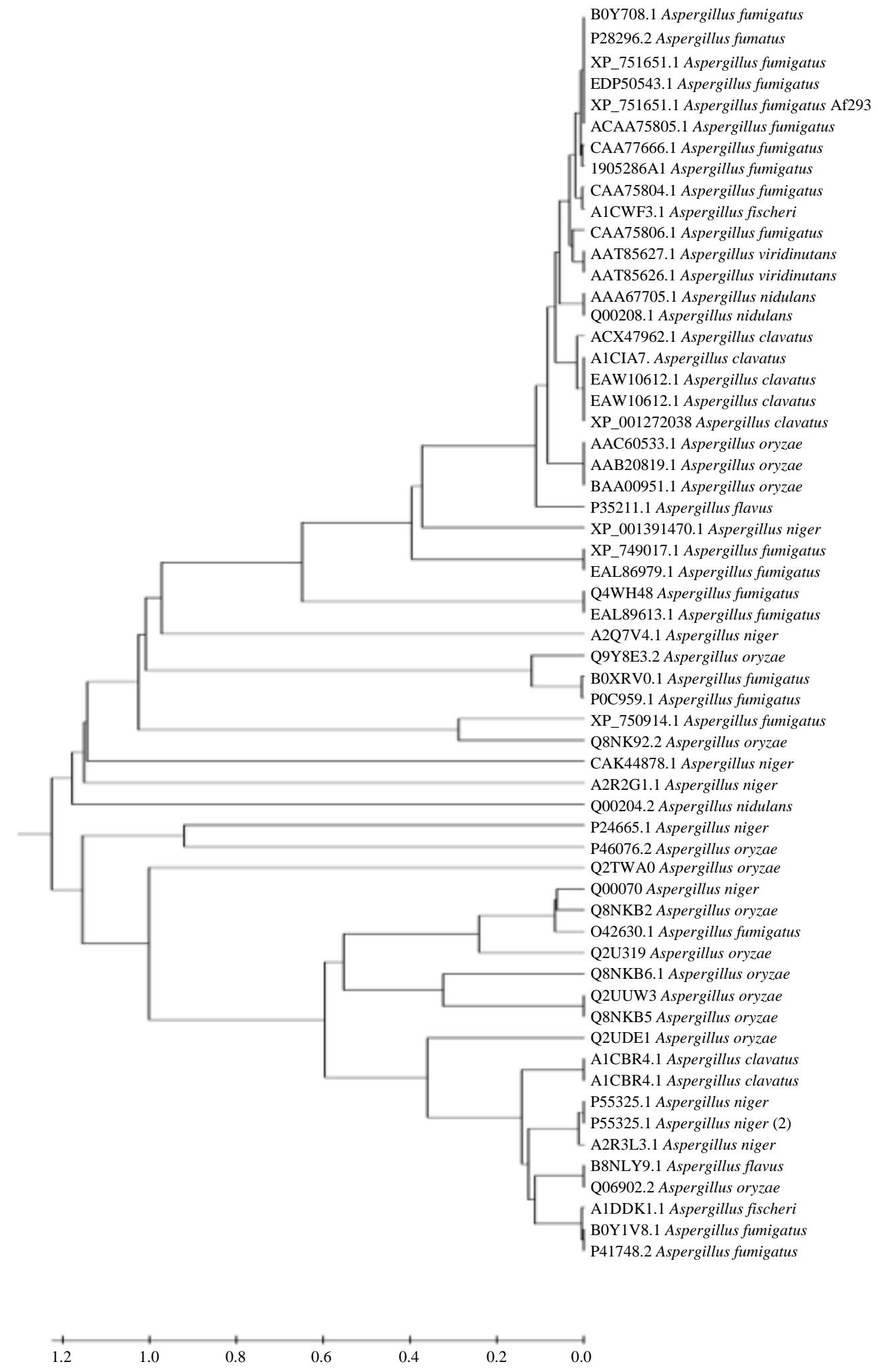

Fig. 1: Phylogenetic tree of amino acid sequence of protease gene isolated from Aspergillus spp. display the location amid the selected gene from NCBI based on amino acid sequence assessments 
1, XP_001272038), 3 Protease gene amino acid sequences isolated from Aspergillus nidulans (Q00208.1, AAA67705.1, Q00204.2), 2 Protease gene amino acid sequences isolated from Aspergillus viridinutans (AAT85626.1, AAT85627.1), 2 Protease gene amino acid sequences isolated from Aspergillus flavus (B8NLY9.1, P35211.1) and 2 Protease gene amino acid sequences isolated from Aspergillus fischeri (A1DDK1.1, A1CWF3.1). Numbers of amino acid bases were varied (400-1030 amino acid bases) from one Aspergillus species to the other.

For molecular phylogenetic construction employing ClustalW, version 1.83 with ncbi database, sequence alignment orientations were performed. A phylogenetic tree was calculated for each protein based on the result of multiple sequence alignments by applying the maximum likelihood method implemented in the Mega 5 software Fig. 1. Moreover, graphic view was displayed based on comparison of some selected amino acid sequences (one of each cluster) to the target sequence protease genes from different Aspergillus sp. The bioinformatics examination appeared that each species of Asperigillus protein normal peptidase superfamily and contains particular space. It has been detailed that cuticle-degrading proteases delivered by a few nematicidal organisms have a place to the peptidase S8 superfamily26-28. To way better get it the work and advancement of this protein, we compared the all grouping with others. Proteases from Aspergillus sp. are isolated into distinctive branches exceptionally clearly, which reflects their diverse sources. The protein's aliphatic index could be a calculation of the relative volume of the subsequent amino acids working by the aliphatic side sequence: Isoleucine, valine, alanine and leucine.

\section{Discussion}

The sequence and structural homology methods primarily evaluate the global similarities between proteins compared to each other (Whisstock and Lesk, 2003). However, the molecular role of a protease in general is limited to its known active site, which may involve an interaction with the binding of protein peptides. Propagations exclusive the thermal permanence of globular proteins are recognized to cover the aliphatic index (Ikai, 1980; Rawlings et al., 2006). The alkaline proteases of the examined Aspergillus species have maintained high aliphatic guide values to be thermostable. The variability guide deals with clarity when planning a protein's in vivo half-life (Guruprasad et al., 1990). Proteins with an in vivo half-life of fewer than $5 \mathrm{~h}$ have been described to demonstration an guide of unpredictability of more than 40, while those with an in vivo half-life of additional than $16 \mathrm{~h}$ (Rogers et al., 1986) have an guide of unpredictability of less than 40. As a consequence, protein comparisons based on global sequence and structural similarity may miss proteins with active sites retained, but divergent sequences and structures may be missing (Powers et al., 2006).

\section{Conclusion}

Characterization of Aspergillus protease protein sequences using bioinformatics programs (in silico) demonstrating the degree of similarity was remarkably found in Aspergillus species for sequence which could be applied for promising cloning of genes putative by designed primers from the conserved regions. Conserved motif amino acid sequence detect the secondary structures.

\section{Acknowledgement}

The authors recognize the funding and facilitation of this research by the City of Scientific Research and Technical Applications, Alexandria, Egypt.

\section{Author Contributions}

All authors equally contributed in this study.

\section{Ethics}

This article is unpublished and includes materials that is unpublished. The corresponding author states that the manuscript has been read and accepted by all the other writers and there are no ethical problems involved.

\section{References}

Anwar, A., \& Saleemuddin, M. (1998). Alkaline proteases: a review. Bioresource technology, 64(3), 175-183.

Asker, M. M., Mahmoud, M. G., El Shebwy, K., \& Abd el Aziz, M. S. (2013). Purification and characterization of two thermostable protease fractions from Bacillus megaterium. Journal of Genetic Engineering and Biotechnology, 11(2), 103-109.

Barrett, A. J. (1994). [1] Classification of peptidases. Methods in enzymology, 244, 1-15.

De Castro, R. J. S., \& Sato, H. H. (2014a). Protease from Aspergillus oryzae: biochemical characterization and application as a potential biocatalyst for production of protein hydrolysates with antioxidant activities. Journal of Food Processing, 2014. 
De Castro, R. J. S., \& Sato, H. H. (2014b). Production and biochemical characterization of protease from Aspergillus oryzae: an evaluation of the physicalchemical parameters using agroindustrial wastes as supports. Biocatalysis and Agricultural Biotechnology, 3(3), 20-25.

Dubey, A. K., Yadav, S., Kumar, M., Singh, V. K., Sarangi, B. K., \& Yadav, D. (2010). In silico characterization of pectate lyase protein sequences from different source organisms. Enzyme Research, 2010.

Gupta, R., Beg, Q., \& Lorenz, P. (2002). Bacterial alkaline proteases: molecular approaches and industrial applications. Applied microbiology and biotechnology, 59(1), 15-32.

Guruprasad, K., Reddy, B. B., \& Pandit, M. W. (1990). Correlation between stability of a protein and its dipeptide composition: a novel approach for predicting in vivo stability of a protein from its primary sequence. Protein Engineering, Design and Selection, 4(2), 155-161.

Heerd, D., Yegin, S., Tari, C., \& Fernandez-Lahore, M. (2012). Pectinase enzyme-complex production by Aspergillus spp. in solid-state fermentation: a comparative study. Food and Bioproducts Processing, 90(2), 102-110.

Ikai, A. (1980). Thermostability and aliphatic index of globular proteins. The Journal of Biochemistry, 88(6), 1895-1898.

Kasana, R. C., Salwan, R., \& Yadav, S. K. (2011). Microbial proteases: detection, production and genetic improvement. Critical reviews in microbiology, 37(3), 262-276.

Li, C., Xu, D., Zhao, M., Sun, L., \& Wang, Y. (2014). Production optimization, purification and characterization of a novel acid protease from a fusant by Aspergillus oryzae and Aspergillus niger. European Food Research and Technology, 238(6), 905-917.

Machida, M., Asai, K., Sano, M., Tanaka, T., Kumagai, T., Terai, G \& Kikuchi, H. (2005). Genome sequencing and analysis of Aspergillus oryzae. Nature, 438(7071), 1157-1161.

Morya, V. K., Yadav, S., Kim, E. K., \& Yadav, D. (2012). In silico characterization of alkaline proteases from different species of Aspergillus. Applied biochemistry and biotechnology, 166(1), 243-257.

Nirmal, N. P., Shankar, S., \& Laxman, R. S. (2011). Fungal proteases: an overview. International Journal of Biotechnology \& Biosciences, 1(1), 1-40.

Nishihira, J., \& Tachikawa, H. (1999). Theoretical Evaluation of a Model of the Catalytic Triads of Serine and Cysteine Proteases byab initioMolecular Orbital Calculation. Journal of theoretical biology, 196(4), 513-519.
Powers, R., Copeland, J. C., Germer, K., Mercier, K. A., Ramanathan, V., \& Revesz, P. (2006). Comparison of protein active site structures for functional annotation of proteins and drug design. Proteins: Structure, Function and Bioinformatics, 65(1), 124-135.

Ramakrishna, V., Rajasekhar, S., \& Reddy, L. S. (2010). Identification and purification of metalloprotease from dry grass pea (Lathyrus sativus L.) seeds. Applied biochemistry and biotechnology, 160(1), 63.

Rawlings, N. D., Morton, F. R., \& Barrett, A. J. (2006). MEROPS: the peptidase database. Nucleic acids research, 34(suppl_1), D270-D272.

Rogers, S., Wells, R., \& Rechsteiner, M. (1986). Amino acid sequences common to rapidly degraded proteins: the PEST hypothesis. Science, 234(4774), 364-368.

Silva, T. A. S., Knob, A., Tremacoldi, C. R., BrochettoBraga, M. R., \& Carmona, E. C. (2011). Purification and some properties of an extracellular acid protease from Aspergillus clavatus. World Journal of Microbiology and Biotechnology, 27(11), 2491-2497.

Tavano, O. L. (2013). Protein hydrolysis using proteases: An important tool for food biotechnology. Journal of Molecular Catalysis B: Enzymatic, 90, 1-11.

Umeadi, C., Kandeel, F., \& Al-Abdullah, I. H. (2008, March). Ulinastatin is a novel protease inhibitor and neutral protease activator. In Transplantation proceedings (Vol. 40, No. 2, pp. 387-389). Elsevier.

Wang, J., Xu, A., Wan, Y., \& Li, Q. (2013). Purification and characterization of a new metallo-neutral protease for beer brewing from Bacillus amyloliquefaciens SYB-001. Applied biochemistry and biotechnology, 170(8), 2021-2033.

Whisstock, J. C., \& Lesk, A. M. (2003). Prediction of protein function from protein sequence and structure. Quarterly reviews of biophysics, 36(3), 307.

Xiao-Lin, Ao., Xi, Yu., Ding-Tao, Wu., Chao, Li., Tong Zhang, Shu-liang, Liu, Shu-Juan, Chen, Li, He, Kang Zhou, \& Li-Kou Zou. (2018). Purification and characterization of neutral protease from Aspergillus oryzae $\mathrm{Y} 1$ isolated from naturally fermented broad beans. AMB Express. 8: 96. https://ambexpress.springeropen.com/articles/10.1186/s13568018-0611-6

Yadav, P. K., Singh, V. K., Yadav, S., Yadav, K. D. S., \& Yadav, D. (2009). In silico analysis of pectin lyase and pectinase sequences. Biochemistry (Moscow), 74(9), 1049-1055.

Yadav, V., Yadav, D., \& Yadav, K. D. S. (2010). In silico analysis of $\alpha$-L-rhamnosidase protein sequences from different source organisms. Online Journal of Bioinformatics, 11(2), 293-301.

Yuzuki, M., Matsushima, K., \& Koyama, Y. (2015). Expression of key hydrolases for soy sauce fermentation in Zygosaccharomyces rouxii. Journal of bioscience and bioengineering, 119(1), 92-94. 
Zhang, H., Zhang, B., Zheng, Y., Shan, A., \& Cheng, B. (2014). Neutral protease expression and optimized conditions for the degradation of blood cells using recombinant Pichia pastoris. International Biodeterioration \& Biodegradation, 93, 235-240.
Zhou, D. Y., Zhu, B. W., Qiao, L., Wu, H. T., Li, D. M., Yang, J. F., \& Murata, Y. (2012). In vitro antioxidant activity of enzymatic hydrolysates prepared from abalone (Haliotis discus hannai Ino) viscera. Food and Bioproducts Processing, 90(2), 148-154.

Table 1: Restriction digestion of protease gene from D00350.1 Aspergillus oryzae

\begin{tabular}{|c|c|c|c|c|c|}
\hline Name & Sequence & Site Length & Overhang & Frequency & Cut positions \\
\hline$\overline{\mathrm{BsaAI}}$ & YACGTR & 6 & blunt & 1 & 1249 \\
\hline BsaBI & GATNNNNATC & 6 & blunt & 1 & 565 \\
\hline BsrBI & CCGCTC & 6 & blunt & 1 & 1061 \\
\hline BtrI & CACGTC & 6 & blunt & 1 & 39 \\
\hline Eco47III & AGCGCT & 6 & blunt & 1 & 1581 \\
\hline MslI & CAYNNNNRTG & 6 & blunt & 1 & 2463 \\
\hline PsiI & TTATAA & 6 & blunt & 1 & 617 \\
\hline PvuII & CAGCTG & 6 & blunt & 1 & 2594 \\
\hline StuI & AGGCCT & 6 & blunt & 1 & 1307 \\
\hline $\mathrm{XmnI}$ & GAANNNNTTC & 6 & blunt & 1 & 1642 \\
\hline AflII & CTTAAG & 6 & five_prime & 1 & 2095 \\
\hline ApoI & RAATTY & 6 & five_prime & 1 & 1718 \\
\hline AvaI & CYCGRG & 6 & five_prime & 1 & 582 \\
\hline BclI & TGATCA & 6 & five_prime & 1 & 2365 \\
\hline BglII & AGATCT & 6 & five_prime & 1 & 1119 \\
\hline Bsp1407I & TGTACA & 6 & five_prime & 1 & 2257 \\
\hline BspHI & TCATGA & 6 & five_prime & 1 & 2422 \\
\hline BspMI & ACCTGC & 6 & five_prime & 1 & 2080 \\
\hline EcoRI & GAATTC & 6 & five_prime & 1 & 1718 \\
\hline Esp3I & CGTCTC & 6 & five_prime & 1 & 1699 \\
\hline HindIII & AAGCTT & 6 & five_prime & 1 & 816 \\
\hline NarI & GGCGCC & 6 & five_prime & 1 & 928 \\
\hline PasI & CCCWGGG & 7 & five_prime & 1 & 1161 \\
\hline PfoI & TCCNGGA & 6 & five_prime & 1 & 821 \\
\hline SpeI & ACTAGT & 6 & five_prime & 1 & 1220 \\
\hline VspI & ATTAAT & 6 & five_prime & 1 & 211 \\
\hline XbaI & TCTAGA & 6 & five_prime & 1 & 2438 \\
\hline XhoI & CTCGAG & 6 & five_prime & 1 & 582 \\
\hline XhoII & RGATCY & 6 & five_prime & 1 & 1119 \\
\hline BfiI & ACTGGG & 6 & three_prime & 1 & 1486 \\
\hline BsmI & GAATGC & 6 & three_prime & 1 & 1642 \\
\hline BtsI & GCAGTG & 6 & three-prime & 1 & 316 \\
\hline Eco57I & CTGAAG & 6 & three_prime & 1 & 629 \\
\hline GsuI & CTGGAG & 6 & three_prime & 1 & 936 \\
\hline PflMI & CCANNNNNTGG & 6 & three_prime & 1 & 2536 \\
\hline SphI & GCATGC & 6 & three_prime & 1 & 550 \\
\hline BsmAI & GTCTC & 5 & five_prime & 2 & 310,1699 \\
\hline BtgZI & GCGATG & 6 & five_prime & 2 & 121,1689 \\
\hline NcoI & CCATGG & 6 & five_prime & 2 & 1348,1933 \\
\hline AatII & GACGTC & 6 & three_prime & 2 & 1858,2044 \\
\hline AloI & GAACNNNNNNTCC & 7 & three_prime & 2 & 359,391 \\
\hline ArsI & GACNNNNNNTTYG & 7 & three_prime & 2 & 2393,2425 \\
\hline $\mathrm{BcgI}$ & CGANNNNNNTGC & 6 & three_prime & 2 & 1205,1239 \\
\hline BglI & GCCNNNNNGGC & 6 & three_prime & 2 & 1304,1403 \\
\hline BsgI & GTGCAG & 6 & three_prime & 2 & 810,2373 \\
\hline Eco57MI & CTGRAG & 6 & three_prime & 2 & 629,936 \\
\hline HaeII & RGCGCY & 6 & three_prime & 2 & 931,1583 \\
\hline HaeIV & GAYNNNNNRTC & 6 & three_prime & 2 & 476,510 \\
\hline KpnI & GGTACC & 6 & three_prime & 2 & 251,1930 \\
\hline PstI & CTGCAG & 6 & three_prime & 2 & 323,2599 \\
\hline SacII & CCGCGG & 6 & three_prime & 2 & 145,772 \\
\hline TaqII & GACCGA & 6 & three_prime & 2 & 1408,1434 \\
\hline TsoI & TARCCA & 6 & three_prime & 2 & 1595,1926 \\
\hline TstI & CACNNNNNNTCC & 6 & three_prime & 2 & 44,76 \\
\hline EcoRV & GATATC & 6 & blunt & 3 & $55,171,1877$ \\
\hline AcyI & GRCGYC & 6 & five_prime & 3 & $928,1855,2041$ \\
\hline CfrI & YGGCCR & 6 & five_prime & 3 & $1345,1754,2029$ \\
\hline SalI & GTCGAC & 6 & five_prime & 3 & $326,1271,2399$ \\
\hline SapI & GCTCTTC & 7 & five_prime & 3 & $1803,1904,2157$ \\
\hline StyI & CCWWGG & 6 & five_prime & 3 & $1348,1933,2035$ \\
\hline
\end{tabular}


Samia Abd Allah Abdal-Aziz and Safaa M. Ali / OnLine Journal of Biological Sciences 2021, 21 (1): 69.119 DOI: 10.3844/ojbsci.2021.69.119

\begin{tabular}{|c|c|c|c|c|c|}
\hline Tth111I & GACNNNGTC & 6 & five_prime & 3 & $2043,2452,2465$ \\
\hline BseMII & CTCAG & 5 & three_prime & 3 & $210,473,802$ \\
\hline BseSI & GKGCMC & 6 & three_prime & 3 & $785,1159,1238$ \\
\hline MmeI & TCCRAC & 6 & three_prime & 3 & $705,1858,2554$ \\
\hline NspI & RCATGY & 6 & three_prime & 3 & $550,630,1334$ \\
\hline TspDTI & ATGAA & 5 & three_prime & 3 & $417,2526,2580$ \\
\hline TspRI & CASTG & 5 & three_prime & 3 & $323,938,1796$ \\
\hline Cfr10I & RCCGGY & 6 & five_prime & 4 & $1226,1319,1675,1752$ \\
\hline HgaI & GACGC & 5 & five_prime & 4 & $313,385,909,2423$ \\
\hline TatI & WGTACW & 6 & five_prime & 4 & $524,829,2257,2557$ \\
\hline Tsp45I & GTSAC & 5 & five_prime & 4 & 86, 493, 836, 2006 \\
\hline BdaI & TGANNNNNNTCA & 6 & three_prime & 4 & $373,407,2402,2436$ \\
\hline BsrI & ACTGG & 5 & three_prime & 4 & $938,1481,1825,2140$ \\
\hline FalI & AAGNNNNNCTT & 6 & three_prime & 4 & $726,758,1864,1896$ \\
\hline Hin4I & GAYNNNNNVTC & 6 & three_prime & 4 & $109,141,477,509$ \\
\hline TspGWI & ACGGA & 5 & three_prime & 4 & $389,559,1444,2196$ \\
\hline FauI & CCCGC & 5 & five_prime & 5 & $149,444,776,807,2006$ \\
\hline SduI & GDGCHC & 6 & three_prime & 5 & $469,785,1159,1202,1238$ \\
\hline TauI & GCSGC & 5 & three_prime & 5 & $147,810,990,1296,1800$ \\
\hline SmlI & CTYRAG & 6 & five_prime & 6 & $263,582,685,1980,2095,2228$ \\
\hline BseRI & GAGGAG & 6 & three_prime & 6 & $171,738,741,1038,1124,1295$ \\
\hline $\mathrm{HphI}$ & GGTGA & 5 & three_prime & 6 & $487,894,951,1448,1553,2360$ \\
\hline AgsI & TTSAA & 5 & three_prime & 7 & $244,510,845,1073,1474,1590,1623$ \\
\hline Hpy99I & CGWCG & 5 & three_prime & 7 & $329,925,1011,1109,2046,2116,2332$ \\
\hline BccI & CCATC & 5 & five_prime & 8 & $649,661,738,1127,1711,1794,1926,1986$ \\
\hline EcoRII & CCWGG & 5 & five_prime & 8 & $821,885,1092,1160,1431,1887,2102,2535$ \\
\hline FokI & GGATG & 5 & five_prime & 9 & $78,127,279,519,617,628,1042,1397,2064$ \\
\hline TfiI & GAWTC & 5 & five_prime & 9 & $23,869,1028,1439,2120,2174,2250,2419,2500$ \\
\hline BbvI & GCAGC & 5 & five_prime & 11 & $333,1185,1303,1458,1468,1507,1659,1961,1988,2074,2581$ \\
\hline TseI & GCWGC & 5 & five_prime & 11 & $321,1173,1316,1446,1481,1520,1672,1974,2001,2062,2594$ \\
\hline MboII & GAAGA & 5 & three_prime & 11 & $112,344,705,936,1053,1162,1549,1820,1850,1891,2174$ \\
\hline SfaNI & GCATC & 5 & five_prime & 13 & $56,144,301,476,541,639,710,1001,1419,1617,1739,1772,2026$ \\
\hline
\end{tabular}

Table 2: Restriction digestion of protease gene from S75278.1 Aspergillus oryzae

\begin{tabular}{|c|c|c|c|c|c|}
\hline Name & Sequence & Site length & Overhang & Frequency & Cut positions \\
\hline BsaAI & YACGTR & 6 & blunt & 1 & 665 \\
\hline EcoRV & GATATC & 6 & blunt & 1 & 1293 \\
\hline PsiI & TTATAA & 6 & blunt & 1 & 36 \\
\hline $\mathrm{XmnI}$ & GAANNNNTTC & 6 & blunt & 1 & 1058 \\
\hline AflII & CTTAAG & 6 & five_prime & 1 & 1511 \\
\hline ApoI & RAATTY & 6 & five_prime & 1 & 1134 \\
\hline AvaI & CYCGRG & 6 & five_prime & 1 & 1 \\
\hline BclI & TGATCA & 6 & five_prime & 1 & 1781 \\
\hline BglII & AGATCT & 6 & five_prime & 1 & 535 \\
\hline BsmAI & GTCTC & 5 & five_prime & 1 & 1115 \\
\hline Bsp1407I & TGTACA & 6 & five_prime & 1 & 1673 \\
\hline BspMI & ACCTGC & 6 & five_prime & 1 & 1496 \\
\hline EcoP15I & CAGCAG & 6 & five_prime & 1 & 639 \\
\hline EcoRI & GAATTC & 6 & five_prime & 1 & 1134 \\
\hline Esp3I & CGTCTC & 6 & five_prime & 1 & 1115 \\
\hline HgaI & GACGC & 5 & five_prime & 1 & 325 \\
\hline NarI & GGCGCC & 6 & five_prime & 1 & 344 \\
\hline PasI & CCCWGGG & 7 & five_prime & 1 & 577 \\
\hline PfoI & TCCNGGA & 6 & five_prime & 1 & 237 \\
\hline PleI & GAGTC & 5 & five_prime & 1 & 1001 \\
\hline SpeI & ACTAGT & 6 & five_prime & 1 & 636 \\
\hline Tth111I & GACNNNGTC & 6 & five_prime & 1 & 1459 \\
\hline XhoI & CTCGAG & 6 & five_prime & 1 & 1 \\
\hline XhoII & RGATCY & 6 & five_prime & 1 & 535 \\
\hline BfiI & ACTGGG & 6 & three_prime & 1 & 902 \\
\hline BseMII & CTCAG & 5 & three_prime & 1 & 218 \\
\hline BsmI & GAATGC & 6 & three_prime & 1 & 1058 \\
\hline BsrDI & GCAATG & 6 & three_prime & 1 & 787 \\
\hline$E \operatorname{co57I}$ & CTGAAG & 6 & three_prime & 1 & 48 \\
\hline GsuI & CTGGAG & 6 & three_prime & 1 & 352 \\
\hline HaeII & RGCGCY & 6 & three_prime & 1 & 347 \\
\hline KpnI & GGTACC & 6 & three_prime & 1 & 1346 \\
\hline
\end{tabular}


Samia Abd Allah Abdal-Aziz and Safaa M. Ali / OnLine Journal of Biological Sciences 2021, 21 (1): 69.119 DOI: 10.3844/ojbsci.2021.69.119

\begin{tabular}{|c|c|c|c|c|c|}
\hline SacII & CCGCGG & 6 & three_prime & 1 & 188 \\
\hline BalI & TGGCCA & 6 & blunt & 2 & 763,1447 \\
\hline NaeI & GCCGGC & 6 & blunt & 2 & 644,1170 \\
\hline BtgZI & GCGATG & 6 & five_prime & 2 & 457,1105 \\
\hline NcoI & CCATGG & 6 & five_prime & 2 & 764,1349 \\
\hline SalI & GTCGAC & 6 & five_prime & 2 & 687,1815 \\
\hline TatI & WGTACW & 6 & five_prime & 2 & 245,1673 \\
\hline Tsp45I & GTSAC & 5 & five_prime & 2 & 252,1422 \\
\hline AatII & GACGTC & 6 & three_prime & 2 & 1274,1460 \\
\hline $\mathrm{BcgI}$ & CGANNNNNNTGC & 6 & three_prime & 2 & 621,655 \\
\hline $\mathrm{BglI}$ & GCCNNNNNGGC & 6 & three_prime & 2 & 720,819 \\
\hline BsgI & GTGCAG & 6 & three_prime & 2 & 226,1789 \\
\hline Eco57MI & CTGRAG & 6 & three_prime & 2 & 48,352 \\
\hline MmeI & TCCRAC & 6 & three_prime & 2 & 121,1274 \\
\hline NspI & RCATGY & 6 & three_prime & 2 & 49,750 \\
\hline TaqII & GACCGA & 6 & three_prime & 2 & 824,850 \\
\hline TspGWI & ACGGA & 5 & three_prime & 2 & 860,1612 \\
\hline TspRI & CASTG & 5 & three_prime & 2 & 354,1212 \\
\hline HindII & GTYRAC & 6 & blunt & 3 & $689,1269,1817$ \\
\hline AccI & GTMKAC & 6 & five_prime & 3 & $688,1682,1816$ \\
\hline AcyI & GRCGYC & 6 & five_prime & 3 & $344,1271,1457$ \\
\hline CfrI & YGGCCR & 6 & five_prime & 3 & $761,1170,1445$ \\
\hline FauI & CCCGC & 5 & five_prime & 3 & $192,223,1422$ \\
\hline SapI & GCTCTTC & 7 & five_prime & 3 & $1219,1320,1573$ \\
\hline StyI & CCWWGG & 6 & five_prime & 3 & $764,1349,1451$ \\
\hline BseSI & GKGCMC & 6 & three_prime & 3 & $201,575,654$ \\
\hline Cfr10I & RCCGGY & 6 & five_prime & 4 & $642,735,1091,1168$ \\
\hline FokI & GGATG & 5 & five_prime & 4 & $36,47,813,1480$ \\
\hline BsrI & ACTGG & 5 & three_prime & 4 & $354,897,1241,1556$ \\
\hline FalI & AAGNNNNNCTT & 6 & three_prime & 4 & $142,174,1280,1312$ \\
\hline SduI & GDGCHC & 6 & three_prime & 4 & $201,575,618,654$ \\
\hline TauI & GCSGC & 5 & three_prime & 4 & $226,406,712,1216$ \\
\hline SmlI & CTYRAG & 6 & five_prime & 5 & $1,101,1396,1511,1644$ \\
\hline Hpy99I & CGWCG & 5 & three_prime & 6 & $341,427,525,1462,1532,1748$ \\
\hline EcoRII & CCWGG & 5 & five_prime & 7 & $237,301,508,576,847,1303,1518$ \\
\hline TfiI & GAWTC & 5 & five_prime & 7 & $285,444,855,986,1536,1590,1666$ \\
\hline $\mathrm{BccI}$ & CCATC & 5 & five_prime & 8 & $68,80,154,543,1127,1210,1342,1402$ \\
\hline SfaNI & GCATC & 5 & five_prime & 8 & $58,126,417,835,1033,1155,1188,1442$ \\
\hline BbvI & GCAGC & 5 & five_prime & 9 & $601,719,874,884,923,1075,1377,1404,1490$ \\
\hline TseI & GCWGC & 5 & five_prime & 9 & $589,732,862,897,936,1088,1390,1417,1478$ \\
\hline MboII & GAAGA & 5 & three_prime & 9 & $121,352,469,578,965,1236,1266,1307,1590$ \\
\hline
\end{tabular}

Table 3: Restriction digestion of protease gene from D10062.1 Aspergillus oryzae

\begin{tabular}{|c|c|c|c|c|c|}
\hline Name & Sequence & Site Length & Overhang & Frequency & Cut Positions \\
\hline$\overline{\mathrm{BsaAI}}$ & YACGTR & 6 & blunt & 1 & 1250 \\
\hline BsaBI & GATNNNNATC & 6 & blunt & 1 & 566 \\
\hline BsrBI & CCGCTC & 6 & blunt & 1 & 1062 \\
\hline BtrI & CACGTC & 6 & blunt & 1 & 39 \\
\hline Eco47III & AGCGCT & 6 & blunt & 1 & 1582 \\
\hline MsII & CAYNNNNRTG & 6 & blunt & 1 & 2464 \\
\hline PsiI & TTATAA & 6 & blunt & 1 & 618 \\
\hline PvuII & CAGCTG & 6 & blunt & 1 & 2595 \\
\hline StuI & AGGCCT & 6 & blunt & 1 & 1308 \\
\hline $\mathrm{XmnI}$ & GAANNNNTTC & 6 & blunt & 1 & 1643 \\
\hline AflII & CTTAAG & 6 & five_prime & 1 & 2096 \\
\hline ApoI & RAATTY & 6 & five_prime & 1 & 1719 \\
\hline AvaI & CYCGRG & 6 & five_prime & 1 & 583 \\
\hline BclI & TGATCA & 6 & five_prime & 1 & 2366 \\
\hline BglII & AGATCT & 6 & five_prime & 1 & 1120 \\
\hline Bsp1407I & TGTACA & 6 & five_prime & 1 & 2258 \\
\hline BspMI & ACCTGC & 6 & five_prime & 1 & 2081 \\
\hline DraII & RGGNCCY & 6 & five_prime & 1 & 153 \\
\hline Esp3I & CGTCTC & 6 & five_prime & 1 & 1700 \\
\hline HindIII & AAGCTT & 6 & five_prime & 1 & 817 \\
\hline NarI & GGCGCC & 6 & five_prime & 1 & 929 \\
\hline PasI & CCCWGGG & 7 & five_prime & 1 & 1162 \\
\hline
\end{tabular}


Samia Abd Allah Abdal-Aziz and Safaa M. Ali / OnLine Journal of Biological Sciences 2021, 21 (1): 69.119 DOI: 10.3844/ojbsci.2021.69.119

\begin{tabular}{|c|c|c|c|c|c|}
\hline PfoI & TCCNGGA & 6 & five_prime & 1 & 822 \\
\hline SpeI & ACTAGT & 6 & five_prime & 1 & 1221 \\
\hline VspI & ATTAAT & 6 & five_prime & 1 & 211 \\
\hline XhoI & CTCGAG & 6 & five_prime & 1 & 583 \\
\hline XhoII & RGATCY & 6 & five_prime & 1 & 1120 \\
\hline BfiI & ACTGGG & 6 & three_prime & 1 & 1487 \\
\hline BsmI & GAATGC & 6 & three_prime & 1 & 1643 \\
\hline Eco57I & CTGAAG & 6 & three_prime & 1 & 630 \\
\hline GsuI & CTGGAG & 6 & three_prime & 1 & 937 \\
\hline PflMI & CCANNNNNTGG & 6 & three_prime & 1 & 2537 \\
\hline SphI & GCATGC & 6 & three_prime & 1 & 551 \\
\hline BalI & TGGCCA & 6 & blunt & 2 & 1348,2032 \\
\hline NaeI & GCCGGC & 6 & blunt & 2 & 1229,1755 \\
\hline AvaII & GGWCC & 5 & five_prime & 2 & 774,1115 \\
\hline BsmAI & GTCTC & 5 & five_prime & 2 & 310,1700 \\
\hline BspHI & TCATGA & 6 & five_prime & 2 & 378,2423 \\
\hline NcoI & CCATGG & 6 & five_prime & 2 & 1349,1934 \\
\hline AatII & GACGTC & 6 & three_prime & 2 & 1859,2045 \\
\hline AloI & GAACNNNNNNTCC & 7 & three_prime & 2 & 359,391 \\
\hline ArsI & GACNNNNNNTTYG & 7 & three_prime & 2 & 2394,2426 \\
\hline $\mathrm{BcgI}$ & CGANNNNNNTGC & 6 & three_prime & 2 & 1206,1240 \\
\hline BglI & GCCNNNNNGGC & 6 & three_prime & 2 & 1305,1404 \\
\hline BsgI & GTGCAG & 6 & three_prime & 2 & 811,2374 \\
\hline Eco57MI & CTGRAG & 6 & three_prime & 2 & 630,937 \\
\hline HaeII & RGCGCY & 6 & three_prime & 2 & 932,1584 \\
\hline HaeIV & GAYNNNNNRTC & 6 & three_prime & 2 & 477,511 \\
\hline KpnI & GGTACC & 6 & three_prime & 2 & 251,1931 \\
\hline PstI & CTGCAG & 6 & three_prime & 2 & 323,2600 \\
\hline SacII & CCGCGG & 6 & three_prime & 2 & 145,773 \\
\hline TaqII & GACCGA & 6 & three_prime & 2 & 1409,1435 \\
\hline TsoI & TARCCA & 6 & three_prime & 2 & 1596,1927 \\
\hline TstI & CACNNNNNNTCC & 6 & three_prime & 2 & 44,76 \\
\hline EcoRV & GATATC & 6 & blunt & 3 & $55,171,1878$ \\
\hline AcyI & GRCGYC & 6 & five_prime & 3 & $929,1856,2042$ \\
\hline CfrI & YGGCCR & 6 & five_prime & 3 & $1346,1755,2030$ \\
\hline StyI & CCWWGG & 6 & five_prime & 3 & $1349,1934,2036$ \\
\hline Tth111I & GACNNNGTC & 6 & five_prime & 3 & $2044,2453,2466$ \\
\hline BseMII & CTCAG & 5 & three_prime & 3 & $210,474,803$ \\
\hline BseSI & GKGCMC & 6 & three_prime & 3 & $786,1160,1239$ \\
\hline BsrDI & GCAATG & 6 & three_prime & 3 & $497,542,1372$ \\
\hline MmeI & TCCRAC & 6 & three_prime & 3 & $706,1859,2555$ \\
\hline NspI & RCATGY & 6 & three_prime & 3 & $551,631,1335$ \\
\hline TspRI & CASTG & 5 & three_prime & 3 & $323,939,1797$ \\
\hline AccI & GTMKAC & 6 & five_prime & 4 & $327,1273,2267,2401$ \\
\hline Cfr10I & RCCGGY & 6 & five_prime & 4 & $1227,1320,1676,1753$ \\
\hline HgaI & GACGC & 5 & five_prime & 4 & $313,386,910,2424$ \\
\hline TatI & WGTACW & 6 & five_prime & 4 & $525,830,2258,2558$ \\
\hline Tsp45I & GTSAC & 5 & five_prime & 4 & $86,494,837,2007$ \\
\hline BdaI & TGANNNNNNTCA & 6 & three_prime & 4 & $374,408,2403,2437$ \\
\hline BsrI & ACTGG & 5 & three_prime & 4 & $939,1482,1826,2141$ \\
\hline FalI & AAGNNNNNCTT & 6 & three_prime & 4 & $727,759,1865,1897$ \\
\hline Hin4I & GAYNNNNNVTC & 6 & three_prime & 4 & $109,141,478,510$ \\
\hline TspDTI & ATGAA & 5 & three_prime & 4 & $367,418,2527,2581$ \\
\hline TspGWI & ACGGA & 5 & three_prime & 4 & $390,560,1445,2197$ \\
\hline HindII & GTYRAC & 6 & blunt & 5 & $328,411,1274,1854,2402$ \\
\hline FauI & CCCGC & 5 & five_prime & 5 & $149,445,777,808,2007$ \\
\hline SduI & GDGCHC & 6 & three_prime & 5 & $470,786,1160,1203,1239$ \\
\hline TauI & GCSGC & 5 & three_prime & 5 & $147,811,991,1297,1801$ \\
\hline SmlI & CTYRAG & 6 & five_prime & 6 & $263,583,686,1981,2096,2229$ \\
\hline BseRI & GAGGAG & 6 & three_prime & 6 & $171,739,742,1039,1125,1296$ \\
\hline HphI & GGTGA & 5 & three_prime & 6 & $488,895,952,1449,1554,2361$ \\
\hline AgsI & TTSAA & 5 & three_prime & 7 & $244,511,846,1074,1475,1591,1624$ \\
\hline Hpy99I & CGWCG & 5 & three_prime & 7 & $329,926,1012,1110,2047,2117,2333$ \\
\hline EcoRII & CCWGG & 5 & five_prime & 8 & $822,886,1093,1161,1432,1888,2103,2536$ \\
\hline BccI & CCATC & 5 & five_prime & 9 & $650,662,739,1128,1712,1795,1927,1987,2322$ \\
\hline FokI & GGATG & 5 & five_prime & 9 & $78,127,279,520,618,629,1043,1398,2065$ \\
\hline TfiI & GAWTC & 5 & five_prime & 9 & $23,870,1029,1440,2121,2175,2251,2420,2501$ \\
\hline BbvI & GCAGC & 5 & five_prime & 11 & $333,1186,1304,1459,1469,1508,1660,1962,1989,2075,2582$ \\
\hline TseI & GCWGC & 5 & five_prime & 11 & $321,1174,1317,1447,1482,1521,1673,1975,2002,2063,2595$ \\
\hline MboII & GAAGA & 5 & three_prime & 11 & $112,344,706,937,1054,1163,1550,1821,1851,1892,2175$ \\
\hline SfaNI & GCATC & 5 & five_prime & 13 & $56,144,301,477,542,640,711,1002,1420,1618,1740,1773,2027$ \\
\hline
\end{tabular}


Samia Abd Allah Abdal-Aziz and Safaa M. Ali / OnLine Journal of Biological Sciences 2021, 21 (1): 69.119 DOI: 10.3844/ojbsci.2021.69.119

\begin{tabular}{|c|c|c|c|c|c|}
\hline Name & Sequence & Site Length & Overhang & Frequency & Cut Positions \\
\hline$\overline{\mathrm{BsaAI}}$ & YACGTR & 6 & blunt & 1 & 665 \\
\hline BsrBI & CCGCTC & 6 & blunt & 1 & 477 \\
\hline EcoRV & GATATC & 6 & blunt & 1 & 1293 \\
\hline NruI & TCGCGA & 6 & blunt & 1 & 991 \\
\hline PsiI & TTATAA & 6 & blunt & 1 & 36 \\
\hline StuI & AGGCCT & 6 & blunt & 1 & 723 \\
\hline $\mathrm{XmnI}$ & GAANNNNTTC & 6 & blunt & 1 & 1058 \\
\hline AflII & CTTAAG & 6 & five_prime & 1 & 1511 \\
\hline AvaI & CYCGRG & 6 & five_prime & 1 & 1 \\
\hline BclI & TGATCA & 6 & five_prime & 1 & 1781 \\
\hline BglII & AGATCT & 6 & five_prime & 1 & 535 \\
\hline BsmAI & GTCTC & 5 & five_prime & 1 & 1115 \\
\hline Bsp1407I & TGTACA & 6 & five_prime & 1 & 1673 \\
\hline BspMI & ACCTGC & 6 & five_prime & 1 & 1496 \\
\hline BtgZI & GCGATG & 6 & five_prime & 1 & 1105 \\
\hline EcoP15I & CAGCAG & 6 & five_prime & 1 & 639 \\
\hline EcoRI & GAATTC & 6 & five_prime & 1 & 1134 \\
\hline Esp3I & CGTCTC & 6 & five_prime & 1 & 1115 \\
\hline HindIII & AAGCTT & 6 & five_prime & 1 & 232 \\
\hline NarI & GGCGCC & 6 & five_prime & 1 & 344 \\
\hline PasI & CCCWGGG & 7 & five_prime & 1 & 577 \\
\hline PfoI & TCCNGGA & 6 & five_prime & 1 & 237 \\
\hline PleI & GAGTC & 5 & five_prime & 1 & 1001 \\
\hline SpeI & ACTAGT & 6 & five_prime & 1 & 636 \\
\hline Tth111I & GACNNNGTC & 6 & five_prime & 1 & 1459 \\
\hline XhoI & CTCGAG & 6 & five_prime & 1 & 1 \\
\hline XhoII & RGATCY & 6 & five_prime & 1 & 535 \\
\hline BfiI & ACTGGG & 6 & three_prime & 1 & 902 \\
\hline BseMII & CTCAG & 5 & three_prime & 1 & 218 \\
\hline BsmI & GAATGC & 6 & three_prime & 1 & 1058 \\
\hline BsrDI & GCAATG & 6 & three_prime & 1 & 787 \\
\hline Eco57I & CTGAAG & 6 & three_prime & 1 & 48 \\
\hline GsuI & CTGGAG & 6 & three_prime & 1 & 352 \\
\hline HaeII & RGCGCY & 6 & three_prime & 1 & 347 \\
\hline KpnI & GGTACC & 6 & three_prime & 1 & 1346 \\
\hline SacII & CCGCGG & 6 & three_prime & 1 & 188 \\
\hline TspGWI & ACGGA & 5 & three_prime & 1 & 1612 \\
\hline BalI & TGGCCA & 6 & blunt & 2 & 763,1447 \\
\hline SalI & GTCGAC & 6 & five_prime & 2 & 687,1815 \\
\hline TatI & WGTACW & 6 & five_prime & 2 & 245,1673 \\
\hline Tsp45I & GTSAC & 5 & five_prime & 2 & 252,1422 \\
\hline AatII & GACGTC & 6 & three_prime & 2 & 1274,1460 \\
\hline BcgI & CGANNNNNNTGC & 6 & three_prime & 2 & 621,655 \\
\hline BglI & GCCNNNNNGGC & 6 & three_prime & 2 & 720,819 \\
\hline BsgI & GTGCAG & 6 & three_prime & 2 & 226,1789 \\
\hline Eco57MI & CTGRAG & 6 & three_prime & 2 & 48,352 \\
\hline MmeI & TCCRAC & 6 & three_prime & 2 & 121,1274 \\
\hline NspI & RCATGY & 6 & three_prime & 2 & 49,750 \\
\hline TaqII & GACCGA & 6 & three_prime & 2 & 824,850 \\
\hline TsoI & TARCCA & 6 & three_prime & 2 & 1011,1342 \\
\hline TspRI & CASTG & 5 & three_prime & 2 & 354,1212 \\
\hline HindII & GTYRAC & 6 & blunt & 3 & $689,1269,1817$ \\
\hline AccI & GTMKAC & 6 & five_prime & 3 & $688,1682,1816$ \\
\hline AcyI & GRCGYC & 6 & five_prime & 3 & $344,1271,1457$ \\
\hline CfrI & YGGCCR & 6 & five_prime & 3 & $761,1170,1445$ \\
\hline FauI & CCCGC & 5 & five_prime & 3 & $192,223,1422$ \\
\hline SapI & GCTCTTC & 7 & five_prime & 3 & $1219,1320,1573$ \\
\hline StyI & CCWWGG & 6 & five_prime & 3 & $764,1349,1451$ \\
\hline BseSI & GKGCMC & 6 & three_prime & 3 & $201,575,654$ \\
\hline Cfr10I & RCCGGY & 6 & five_prime & 4 & $642,735,1091,1168$ \\
\hline BsrI & ACTGG & 5 & three_prime & 4 & $354,897,1241,1556$ \\
\hline FalI & AAGNNNNNCTT & 6 & three_prime & 4 & $142,174,1280,1312$ \\
\hline SduI & GDGCHC & 6 & three_prime & 4 & $201,575,618,654$ \\
\hline TauI & GCSGC & 5 & three_prime & 4 & $226,406,712,1216$ \\
\hline FokI & GGATG & 5 & five_prime & 5 & $36,47,458,813,1480$ \\
\hline SmlI & CTYRAG & 6 & five_prime & 5 & $1,101,1396,1511,1644$ \\
\hline AgsI & TTSAA & 5 & three_prime & 5 & $261,489,890,1006,1039$ \\
\hline BseRI & GAGGAG & 6 & three_prime & 5 & $154,157,454,540,711$ \\
\hline HphI & GGTGA & 5 & three_prime & 5 & $310,367,864,969,1776$ \\
\hline Нpy99I & CGWCG & 5 & three_prime & 6 & $341,427,525,1462,1532,1748$ \\
\hline EcoRII & CCWGG & 5 & five_prime & 7 & $237,301,508,576,847,1303,1518$ \\
\hline TfiI & GAWTC & 5 & five_prime & 7 & $285,444,855,986,1536,1590,1666$ \\
\hline BccI & CCATC & 5 & five_prime & 8 & $68,80,154,543,1127,1210,1342,1402$ \\
\hline SfaNI & GCATC & 5 & five_prime & 8 & $58,126,417,835,1033,1155,1188,1442$ \\
\hline BbvI & GCAGC & 5 & five_prime & 9 & $601,719,874,884,923,1075,1377,1404,1490$ \\
\hline TseI & GCWGC & 5 & five_prime & 9 & $589,732,862,897,936,1088,1390,1417,1478$ \\
\hline MboII & GAAGA & 5 & three_prime & 9 & $121,352,469,578,965,1236,1266,1307,1590$ \\
\hline
\end{tabular}


Samia Abd Allah Abdal-Aziz and Safaa M. Ali / OnLine Journal of Biological Sciences 2021, 21 (1): 69.119 DOI: 10.3844/ojbsci.2021.69.119

Table 5: Restriction digestion of protease gene from X17561.1 A. oryzae

\begin{tabular}{|c|c|c|c|c|c|}
\hline Name & Sequence & Site length & Overhang & Frequency & Cut positions \\
\hline & YACGTR & 6 & blunt & 1 & 529 \\
\hline EcoRV & GATATC & 6 & blunt & 1 & 1042 \\
\hline StuI & AGGCCT & 6 & blunt & 1 & 587 \\
\hline $\mathrm{XmnI}$ & GAANNNNTTC & 6 & blunt & 1 & 863 \\
\hline AflII & CTTAAG & 6 & five_prime & 1 & 1260 \\
\hline BglII & AGATCT & 6 & five_prime & 1 & 399 \\
\hline Bsp1407I & TGTACA & 6 & five_prime & 1 & 1422 \\
\hline BspMI & ACCTGC & 6 & five_prime & 1 & 1245 \\
\hline EcoP15I & CAGCAG & 6 & five_prime & 1 & 503 \\
\hline HgaI & GACGC & 5 & five_prime & 1 & 239 \\
\hline HindIII & AAGCTT & 6 & five_prime & 1 & 146 \\
\hline NarI & GGCGCC & 6 & five_prime & 1 & 258 \\
\hline PasI & CCCWGGG & 7 & five_prime & 1 & 441 \\
\hline PfoI & TCCNGGA & 6 & five_prime & 1 & 151 \\
\hline SalI & GTCGAC & 6 & five_prime & 1 & 551 \\
\hline SpeI & ACTAGT & 6 & five_prime & 1 & 500 \\
\hline Tth111I & GACNNNGTC & 6 & five_prime & 1 & 1208 \\
\hline XhoII & RGATCY & 6 & five_prime & 1 & 399 \\
\hline BsgI & GTGCAG & 6 & three_prime & 1 & 140 \\
\hline BsmI & GAATGC & 6 & three_prime & 1 & 863 \\
\hline BsrDI & GCAATG & 6 & three_prime & 1 & 651 \\
\hline Eco57MI & CTGRAG & 6 & three_prime & 1 & 266 \\
\hline GsuI & CTGGAG & 6 & three_prime & 1 & 266 \\
\hline HaeII & RGCGCY & 6 & three_prime & 1 & 261 \\
\hline KpnI & GGTACC & 6 & three_prime & 1 & 1095 \\
\hline NspI & RCATGY & 6 & three_prime & 1 & 614 \\
\hline SacII & CCGCGG & 6 & three_prime & 1 & 102 \\
\hline BalI & TGGCCA & 6 & blunt & 2 & 627,1196 \\
\hline HindII & GTYRAC & 6 & blunt & 2 & 553,1018 \\
\hline NaeI & GCCGGC & 6 & blunt & 2 & 508,919 \\
\hline AccI & GTMKAC & 6 & five_prime & 2 & 552,1431 \\
\hline AvaII & GGWCC & 5 & five_prime & 2 & 103,394 \\
\hline FokI & GGATG & 5 & five_prime & 2 & 677,1229 \\
\hline Tsp45I & GTSAC & 5 & five_prime & 2 & 166,1171 \\
\hline AatII & GACGTC & 6 & three_prime & 2 & 1023,1209 \\
\hline BglI & GCCNNNNNGGC & 6 & three_prime & 2 & 584,683 \\
\hline MmeI & TCCRAC & 6 & three_prime & 2 & 35,1023 \\
\hline TaqII & GACCGA & 6 & three_prime & 2 & 688,714 \\
\hline TsoI & TARCCA & 6 & three_prime & 2 & 816,1091 \\
\hline TspGWI & ACGGA & 5 & three_prime & 2 & 724,1361 \\
\hline TspRI & CASTG & 5 & three_prime & 2 & 268,961 \\
\hline AcyI & GRCGYC & 6 & five_prime & 3 & $258,1020,1206$ \\
\hline CfrI & YGGCCR & 6 & five_prime & 3 & $625,919,1194$ \\
\hline FauI & CCCGC & 5 & five_prime & 3 & $106,137,1171$ \\
\hline SapI & GCTCTTC & 7 & five_prime & 3 & $968,1069,1322$ \\
\hline StyI & CCWWGG & 6 & five_prime & 3 & $628,1098,1200$ \\
\hline AgsI & TTSAA & 5 & three_prime & 3 & $175,754,844$ \\
\hline BseSI & GKGCMC & 6 & three_prime & 3 & $115,439,518$ \\
\hline HphI & GGTGA & 5 & three_prime & 3 & $224,281,728$ \\
\hline Cfr10I & RCCGGY & 6 & five_prime & 4 & $506,599,896,917$ \\
\hline SmlI & CTYRAG & 6 & five_prime & 4 & $15,1145,1260,1393$ \\
\hline $\mathrm{BcgI}$ & CGANNNNNNTGC & 6 & three_prime & 4 & $359,393,485,519$ \\
\hline BsrI & ACTGG & 5 & three_prime & 4 & $268,761,990,1305$ \\
\hline FalI & AAGNNNNNCTT & 6 & three_prime & 4 & $56,88,1029,1061$ \\
\hline SduI & GDGCHC & 6 & three_prime & 4 & $115,439,482,518$ \\
\hline TauI & GCSGC & 5 & three_prime & 4 & $140,320,576,965$ \\
\hline BccI & CCATC & 5 & five_prime & 5 & $68,407,959,1091,1151$ \\
\hline BseRI & GAGGAG & 6 & three_prime & 5 & $68,71,368,404,575$ \\
\hline Нpy99I & CGWCG & 5 & three_prime & 5 & $255,341,389,1211,1281$ \\
\hline EcoRII & CCWGG & 5 & five_prime & 6 & $151,215,440,711,1052,1267$ \\
\hline TfiI & GAWTC & 5 & five_prime & 6 & $199,358,719,1285,1339,1415$ \\
\hline SfaNI & GCATC & 5 & five_prime & 7 & $40,331,699,838,904,937,1191$ \\
\hline MboII & GAAGA & 5 & three_prime & 8 & $35,266,383,442,985,1015,1056,1339$ \\
\hline BbvI & GCAGC & 5 & five_prime & 9 & $465,583,738,748,787,880,1126,1153,1239$ \\
\hline TseI & GCWGC & 5 & five_prime & 9 & $453,596,726,761,800,893,1139,1166,1227$ \\
\hline
\end{tabular}


Samia Abd Allah Abdal-Aziz and Safaa M. Ali / OnLine Journal of Biological Sciences 2021, 21 (1): 69.119 DOI: 10.3844/ojbsci.2021.69.119

Table 6: Restriction digestion of protease gene from AB807664.1 Aspergillus fumigatus

\begin{tabular}{|c|c|c|c|c|c|}
\hline Name & Sequence & Site length & Overhang & Frequency & Cut positions \\
\hline$\overline{\text { BalI }}$ & TGGCCA & 6 & blunt & 1 & 181 \\
\hline BsrBI & CCGCTC & 6 & blunt & 1 & 583 \\
\hline BtrI & CACGTC & 6 & blunt & 1 & 63 \\
\hline Eco47III & AGCGCT & 6 & blunt & 1 & 9 \\
\hline EcoRV & GATATC & 6 & blunt & 1 & 684 \\
\hline $\mathrm{XmnI}$ & GAANNNNTTC & 6 & blunt & 1 & 433 \\
\hline AflIII & ACRYGT & 6 & five_prime & 1 & 539 \\
\hline BspMI & ACCTGC & 6 & five_prime & 1 & 512 \\
\hline BtgZI & GCGATG & 6 & five_prime & 1 & 68 \\
\hline EcoP15I & CAGCAG & 6 & five_prime & 1 & 436 \\
\hline FauI & CCCGC & 5 & five_prime & 1 & 634 \\
\hline FokI & GGATG & 5 & five_prime & 1 & 229 \\
\hline PleI & GAGTC & 5 & five_prime & 1 & 231 \\
\hline StyI & CCWWGG & 6 & five_prime & 1 & 321 \\
\hline BciVI & GTATCC & 6 & three_prime & 1 & 496 \\
\hline BfiI & ACTGGG & 6 & three_prime & 1 & 270 \\
\hline BsrDI & GCAATG & 6 & three_prime & 1 & 516 \\
\hline HaeII & RGCGCY & 6 & three_prime & 1 & 11 \\
\hline KpnI & GGTACC & 6 & three_prime & 1 & 155 \\
\hline NspI & RCATGY & 6 & three_prime & 1 & 543 \\
\hline TsoI & TARCCA & 6 & three_prime & 1 & 386 \\
\hline TspRI & CASTG & 5 & three_prime & 1 & 48 \\
\hline AccI & GTMKAC & 6 & five_prime & 2 & 38,659 \\
\hline BccI & CCATC & 5 & five_prime & 2 & 247,371 \\
\hline CfrI & YGGCCR & 6 & five_prime & 2 & 129,179 \\
\hline EcoRII & CCWGG & 5 & five_prime & 2 & 215,694 \\
\hline SalI & GTCGAC & 6 & five_prime & 2 & 37,658 \\
\hline BaeI & ACNNNNGTAYC & 7 & three_prime & 2 & 468,501 \\
\hline BsrI & ACTGG & 5 & three_prime & 2 & 265,358 \\
\hline FalI & AAGNNNNNCTT & 6 & three_prime & 2 & 434,466 \\
\hline Hin4I & GAYNNNNNVTC & 6 & three_prime & 2 & 273,305 \\
\hline Нру99I & CGWCG & 5 & three_prime & 2 & 67,463 \\
\hline MmeI & TCCRAC & 6 & three_prime & 2 & 511,665 \\
\hline TspDTI & ATGAA & 5 & three_prime & 2 & 455,527 \\
\hline AgsI & TTSAA & 5 & three_prime & 3 & $258,351,414$ \\
\hline MboII & GAAGA & 5 & three_prime & 3 & $198,204,657$ \\
\hline HindII & GTYRAC & 6 & blunt & 4 & $39,57,120,660$ \\
\hline BbvI & GCAGC & 5 & five_prime & 4 & $122,291,450,591$ \\
\hline SfaNI & GCATC & 5 & five_prime & 4 & $56,91,134,546$ \\
\hline TseI & GCWGC & 5 & five_prime & 4 & $110,304,463,604$ \\
\hline TauI & GCSGC & 5 & three_prime & 4 & $80,103,164,374$ \\
\hline TspGWI & ACGGA & 5 & three_prime & 4 & $149,188,192,642$ \\
\hline
\end{tabular}

Table 7: Restriction digestion of protease gene from X66935.1 Aspergillus.fumigatus

\begin{tabular}{|c|c|c|c|c|c|}
\hline Name & Sequence & Site length & Overhang & Frequency & Cut positions \\
\hline$\overline{\text { BalI }}$ & TGGCCA & 6 & blunt & 1 & 199 \\
\hline BsrBI & CCGCTC & 6 & blunt & 1 & 602 \\
\hline BtrI & CACGTC & 6 & blunt & 1 & 81 \\
\hline Eco47III & AGCGCT & 6 & blunt & 1 & 27 \\
\hline $\mathrm{XmnI}$ & GAANNNNTTC & 6 & blunt & 1 & 452 \\
\hline AflIII & ACRYGT & 6 & five_prime & 1 & 558 \\
\hline BspMI & ACCTGC & 6 & five_prime & 1 & 531 \\
\hline BtgZI & GCGATG & 6 & five_prime & 1 & 86 \\
\hline EcoP15I & CAGCAG & 6 & five_prime & 1 & 455 \\
\hline FauI & CCCGC & 5 & five_prime & 1 & 653 \\
\hline FokI & GGATG & 5 & five_prime & 1 & 247 \\
\hline PleI & GAGTC & 5 & five_prime & 1 & 249 \\
\hline StyI & CCWWGG & 6 & five_prime & 1 & 339 \\
\hline BciVI & GTATCC & 6 & three_prime & 1 & 515 \\
\hline BfiI & ACTGGG & 6 & three_prime & 1 & 288 \\
\hline BtsI & GCAGTG & 6 & three_prime & 1 & 729 \\
\hline HaeII & RGCGCY & 6 & three_prime & 1 & 29 \\
\hline KpnI & GGTACC & 6 & three_prime & 1 & 173 \\
\hline TsoI & TARCCA & 6 & three_prime & 1 & 405 \\
\hline TspDTI & ATGAA & 5 & three_prime & 1 & 474 \\
\hline AccI & GTMKAC & 6 & five_prime & 2 & 56,678 \\
\hline EcoRII & CCWGG & 5 & five_prime & 2 & 233,713 \\
\hline SalI & GTCGAC & 6 & five_prime & 2 & 55,677 \\
\hline BaeI & ACNNNNGTAYC & 7 & three_prime & 2 & 487,520 \\
\hline BsrI & ACTGG & 5 & three_prime & 2 & 283,376 \\
\hline FalI & AAGNNNNNCTT & 6 & three_prime & 2 & 453,485 \\
\hline Hin4I & GAYNNNNNVTC & 6 & three_prime & 2 & 291,323 \\
\hline Нpy99I & CGWCG & 5 & three_prime & 2 & 85,482 \\
\hline MmeI & TCCRAC & 6 & three_prime & 2 & 530,684 \\
\hline
\end{tabular}


Samia Abd Allah Abdal-Aziz and Safaa M. Ali / OnLine Journal of Biological Sciences 2021, 21 (1): 69.119 DOI: 10.3844/ojbsci.2021.69.119

\begin{tabular}{|c|c|c|c|c|c|}
\hline TspRI & CASTG & 5 & three_prime & 2 & 66,736 \\
\hline BccI & CCATC & 5 & five_prime & 3 & $265,390,752$ \\
\hline CfrI & YGGCCR & 6 & five_prime & 3 & $30,147,197$ \\
\hline MboII & GAAGA & 5 & three_prime & 3 & $216,222,676$ \\
\hline HindII & GTYRAC & 6 & blunt & 4 & $57,75,138,679$ \\
\hline BbvI & GCAGC & 5 & five_prime & 4 & $140,309,469,610$ \\
\hline SfaNI & GCATC & 5 & five_prime & 4 & $74,109,152,565$ \\
\hline TauI & GCSGC & 5 & three_prime & 4 & $98,121,182,393$ \\
\hline TspGWI & ACGGA & 5 & three_prime & 4 & $167,206,210,661$ \\
\hline
\end{tabular}

Table 8: Restriction digestion of protease gene from Z11580.1 Aspergillus.fumigatus

\begin{tabular}{|c|c|c|c|c|c|}
\hline Name & Sequence & Site length & Overhang & Frequency & Cut positions \\
\hline BsaBI & GATNNNNATC & 6 & blunt & 1 & 1940 \\
\hline BtrI & CACGTC & 6 & blunt & 1 & 860 \\
\hline Eco47III & AGCGCT & 6 & blunt & 1 & 806 \\
\hline EcoRV & GATATC & 6 & blunt & 1 & 1478 \\
\hline MsII & CAYNNNNRTG & 6 & blunt & 1 & 142 \\
\hline PsiI & TTATAA & 6 & blunt & 1 & 195 \\
\hline ScaI & AGTACT & 6 & blunt & 1 & 626 \\
\hline $\mathrm{XmnI}$ & GAANNNNTTC & 6 & blunt & 1 & 1227 \\
\hline AclI & AACGTT & 6 & five_prime & 1 & 1654 \\
\hline AflII & CTTAAG & 6 & five_prime & 1 & 1696 \\
\hline AflIII & ACRYGT & 6 & five_prime & 1 & 1333 \\
\hline ApaLI & GTGCAC & 6 & five_prime & 1 & 670 \\
\hline AscI & GGCGCGCC & 8 & five_prime & 1 & 355 \\
\hline AvaII & GGWCC & 5 & five_prime & 1 & 695 \\
\hline BglII & AGATCT & 6 & five_prime & 1 & 218 \\
\hline BsePI & GCGCGC & 6 & five_prime & 1 & 355 \\
\hline DraII & RGGNCCY & 6 & five_prime & 1 & 746 \\
\hline Esp3I & CGTCTC & 6 & five_prime & 1 & 610 \\
\hline PfoI & TCCNGGA & 6 & five_prime & 1 & 364 \\
\hline XhoII & RGATCY & 6 & five_prime & 1 & 218 \\
\hline AatII & GACGTC & 6 & three_prime & 1 & 622 \\
\hline AlwNI & CAGNNNCTG & 6 & three_prime & 1 & 289 \\
\hline BciVI & GTATCC & 6 & three_prime & 1 & 1290 \\
\hline BfiI & ACTGGG & 6 & three_prime & 1 & 1067 \\
\hline BseRI & GAGGAG & 6 & three_prime & 1 & 705 \\
\hline BsmI & GAATGC & 6 & three_prime & 1 & 69 \\
\hline BsrI & ACTGG & 5 & three_prime & 1 & 1062 \\
\hline DrdI & GACNNNNNNGTC & 6 & three_prime & 1 & 240 \\
\hline HaeIV & GAYNNNNNRTC & 6 & three_prime & 1 & 286 \\
\hline HphI & GGTGA & 5 & three_prime & 1 & 129 \\
\hline PstI & CTGCAG & 6 & three_prime & 1 & 1607 \\
\hline TsoI & TARCCA & 6 & three_prime & 1 & 1180 \\
\hline $\mathrm{XcmI}$ & CCANNNNNNNNNTGG & 6 & three_prime & 1 & 1760 \\
\hline BsrBI & CCGCTC & 6 & blunt & 2 & 1377,1614 \\
\hline AccI & GTMKAC & 6 & five_prime & 2 & 835,1453 \\
\hline BseYI & CCCAGC & 6 & five_prime & 2 & 254,639 \\
\hline NcoI & CCATGG & 6 & five_prime & 2 & 741,1534 \\
\hline SalI & GTCGAC & 6 & five_prime & 2 & 834,1452 \\
\hline SapI & GCTCTTC & 7 & five_prime & 2 & 531,2091 \\
\hline AlfI & GCANNNNNNTGC & 6 & three_prime & 2 & 302,336 \\
\hline ArsI & GACNNNNNNTTYG & 7 & three_prime & 2 & 508,540 \\
\hline BaeI & ACNNNNGTAYC & 7 & three_prime & 2 & 1262,1295 \\
\hline BplI & GAGNNNNNCTC & 6 & three_prime & 2 & 1575,1607 \\
\hline BseMII & CTCAG & 5 & three_prime & 2 & 123,399 \\
\hline BseSI & GKGCMC & 6 & three_prime & 2 & 429,674 \\
\hline BstXI & CCANNNNNNTGG & 6 & three_prime & 2 & 1760,2091 \\
\hline Eco57I & CTGAAG & 6 & three_prime & 2 & 178,615 \\
\hline FalI & AAGNNNNNCTT & 6 & three_prime & 2 & 1228,1260 \\
\hline GsuI & CTGGAG & 6 & three_prime & 2 & 197,510 \\
\hline HaeII & RGCGCY & 6 & three_prime & 2 & 739,808 \\
\hline KpnI & GGTACC & 6 & three_prime & 2 & 710,952 \\
\hline SphI & GCATGC & 6 & three_prime & 2 & 5,2162 \\
\hline BalI & TGGCCA & 6 & blunt & 3 & $978,1539,1632$ \\
\hline BtgZI & GCGATG & 6 & five_prime & 3 & $45,865,2072$ \\
\hline SmlI & CTYRAG & 6 & five_prime & 3 & $1581,1696,2034$ \\
\hline TatI & WGTACW & 6 & five_prime & 3 & $403,624,1567$ \\
\hline TfiI & GAWTC & 5 & five_prime & 3 & $128,392,1801$ \\
\hline Tsp45I & GTSAC & 5 & five_prime & 3 & $117,410,1607$ \\
\hline
\end{tabular}


Samia Abd Allah Abdal-Aziz and Safaa M. Ali / OnLine Journal of Biological Sciences 2021, 21 (1): 69.119 DOI: 10.3844/ojbsci.2021.69.119

\begin{tabular}{|c|c|c|c|c|c|}
\hline BtsI & GCAGTG & 6 & three_prime & 3 & $64,1504,1612$ \\
\hline HindII & GTYRAC & 6 & blunt & 4 & $836,854,917,1454$ \\
\hline AcyI & GRCGYC & 6 & five_prime & 4 & $586,619,715,736$ \\
\hline PleI & GAGTC & 5 & five_prime & 4 & $294,455,1028,1753$ \\
\hline BsaXI & ACNNNNNCTCC & 6 & three_prime & 4 & $558,588,1506,1536$ \\
\hline BsrDI & GCAATG & 6 & three_prime & 4 & $50,1310,1697,1838$ \\
\hline Eco57MI & CTGRAG & 6 & three_prime & 4 & $178,197,510,615$ \\
\hline SduI & GDGCHC & 6 & three_prime & 4 & $429,674,1783,1813$ \\
\hline EcoRII & CCWGG & 5 & five_prime & 5 & $364,395,748,1012,1488$ \\
\hline HgaI & GACGC & 5 & five_prime & 5 & $483,594,723,2011,2054$ \\
\hline StyI & CCWWGG & 6 & five_prime & 5 & $326,741,1118,1534,2084$ \\
\hline AgsI & TTSAA & 5 & three_prime & 5 & $419,1055,1146,1208,1952$ \\
\hline MmeI & TCCRAC & 6 & three_prime & 5 & $44,269,1305,1459,1884$ \\
\hline BsmAI & GTCTC & 5 & five_prime & 6 & $100,112,176,454,610,2037$ \\
\hline FokI & GGATG & 5 & five_prime & 6 & $86,1026,1772,1948,2002,2094$ \\
\hline $\mathrm{BcgI}$ & CGANNNNNNTGC & 6 & three_prime & 6 & $389,423,1884,1918,2062,2096$ \\
\hline TauI & GCSGC & 5 & three_prime & 6 & $514,564,877,900,961,1168$ \\
\hline TspDTI & ATGAA & 5 & three_prime & 6 & $154,181,1249,1321,2075,2096$ \\
\hline SfaNI & GCATC & 5 & five_prime & 7 & $22,277,853,888,931,1340,1627$ \\
\hline Нpy99I & CGWCG & 5 & three_prime & 7 & $25,379,499,528,864,1257,2048$ \\
\hline TspRI & CASTG & 5 & three_prime & 7 & $71,473,845,1511,1612,1988,2074$ \\
\hline TspGWI & ACGGA & 5 & three_prime & 8 & $946,985,989,1436,1553,1798,1948,2100$ \\
\hline BbvI & GCAGC & 5 & five_prime & 9 & $307,368,766,919,1088,1244,1385,1589,1675$ \\
\hline TseI & GCWGC & 5 & five_prime & 9 & $320,381,754,907,1101,1257,1398,1602,1663$ \\
\hline BccI & CCATC & 5 & five_prime & 10 & $107,600,1044,1165,1527,1568,1750,1754,1921,1930$ \\
\hline MboII & GAAGA & 5 & three_prime & 12 & $171,191,401,465,548,621,995,1001,1451,1722,2074,2108$ \\
\hline
\end{tabular}

Table 9: Restriction digestion of protease gene from XM_746558.1 Aspergillus fumigatus Af293

\begin{tabular}{|c|c|c|c|c|c|}
\hline Name & Sequence & Site length & Overhang & Frequency & Cut positions \\
\hline$\overline{\text { BtrI }}$ & CACGTC & 6 & blunt & 1 & 507 \\
\hline Eco47III & AGCGCT & 6 & blunt & 1 & 453 \\
\hline EcoRV & GATATC & 6 & blunt & 1 & 990 \\
\hline $\mathrm{XmnI}$ & GAANNNNTTC & 6 & blunt & 1 & 811 \\
\hline AclI & AACGTT & 6 & five_prime & 1 & 1166 \\
\hline AscI & GGCGCGCC & 8 & five_prime & 1 & 59 \\
\hline AvaII & GGWCC & 5 & five_prime & 1 & 342 \\
\hline BsePI & GCGCGC & 6 & five_prime & 1 & 59 \\
\hline BsmAI & GTCTC & 5 & five_prime & 1 & 158 \\
\hline BtgZI & GCGATG & 6 & five_prime & 1 & 512 \\
\hline DraII & RGGNCCY & 6 & five_prime & 1 & 393 \\
\hline FauI & CCCGC & 5 & five_prime & 1 & 940 \\
\hline FokI & GGATG & 5 & five_prime & 1 & 673 \\
\hline HindIII & AAGCTT & 6 & five_prime & 1 & 1186 \\
\hline NarI & GGCGCC & 6 & five_prime & 1 & 383 \\
\hline PfoI & TCCNGGA & 6 & five_prime & 1 & 68 \\
\hline SapI & GCTCTTC & 7 & five_prime & 1 & 235 \\
\hline SmlI & CTYRAG & 6 & five_prime & 1 & 1093 \\
\hline BfiI & ACTGGG & 6 & three_prime & 1 & 714 \\
\hline BseMII & CTCAG & 5 & three_prime & 1 & 103 \\
\hline BseRI & GAGGAG & 6 & three_prime & 1 & 352 \\
\hline BseSI & GKGCMC & 6 & three_prime & 1 & 133 \\
\hline BsrDI & GCAATG & 6 & three_prime & 1 & 1209 \\
\hline BsrI & ACTGG & 5 & three_prime & 1 & 709 \\
\hline Eco57MI & CTGRAG & 6 & three_prime & 1 & 214 \\
\hline GsuI & CTGGAG & 6 & three_prime & 1 & 214 \\
\hline MmeI & TCCRAC & 6 & three_prime & 1 & 971 \\
\hline PstI & CTGCAG & 6 & three_prime & 1 & 1119 \\
\hline SduI & GDGCHC & 6 & three_prime & 1 & 133 \\
\hline TsoI & TARCCA & 6 & three_prime & 1 & 764 \\
\hline TspDTI & ATGAA & 5 & three_prime & 1 & 833 \\
\hline BsrBI & CCGCTC & 6 & blunt & 2 & 889,1126 \\
\hline EcoP15I & CAGCAG & 6 & five_prime & 2 & 814,1084 \\
\hline NcoI & CCATGG & 6 & five_prime & 2 & 388,1046 \\
\hline PleI & GAGTC & 5 & five_prime & 2 & 159,675 \\
\hline SalI & GTCGAC & 6 & five_prime & 2 & 481,964 \\
\hline $\mathrm{BcgI}$ & CGANNNNNNTGC & 6 & three_prime & 2 & 93,127 \\
\hline BplI & GAGNNNNNCTC & 6 & three_prime & 2 & 1087,1119 \\
\hline BtsI & GCAGTG & 6 & three_prime & 2 & 1016,1124 \\
\hline FalI & AAGNNNNNCTT & 6 & three_prime & 2 & 812,844 \\
\hline
\end{tabular}


Samia Abd Allah Abdal-Aziz and Safaa M. Ali / OnLine Journal of Biological Sciences 2021, 21 (1): 69.119 DOI: 10.3844/ojbsci.2021.69.119

\begin{tabular}{|c|c|c|c|c|c|}
\hline HaeII & RGCGCY & 6 & three_prime & 2 & 386,455 \\
\hline Hin4I & GAYNNNNNVTC & 6 & three_prime & 2 & 717,749 \\
\hline KpnI & GGTACC & 6 & three_prime & 2 & 357,599 \\
\hline AccI & GTMKAC & 6 & five_prime & 3 & $482,965,1079$ \\
\hline AcyI & GRCGYC & 6 & five_prime & 3 & $290,362,383$ \\
\hline HgaI & GACGC & 5 & five_prime & 3 & $187,298,370$ \\
\hline AgsI & TTSAA & 5 & three_prime & 3 & $123,702,792$ \\
\hline BccI & CCATC & 5 & five_prime & 4 & $304,691,1039,1080$ \\
\hline CfrI & YGGCCR & 6 & five_prime & 4 & $573,623,1049,1142$ \\
\hline StyI & CCWWGG & 6 & five_prime & 4 & $30,388,765,1046$ \\
\hline BsaXI & ACNNNNNCTCC & 6 & three_prime & 4 & $262,292,1018,1048$ \\
\hline EcoRII & CCWGG & 5 & five_prime & 5 & $68,99,395,659,1000$ \\
\hline SfaNI & GCATC & 5 & five_prime & 5 & $500,535,578,852,1139$ \\
\hline Нpy99I & CGWCG & 5 & three_prime & 5 & $83,203,232,511,841$ \\
\hline TauI & GCSGC & 5 & three_prime & 5 & $218,268,524,547,608$ \\
\hline TspGWI & ACGGA & 5 & three_prime & 5 & $593,632,636,948,1065$ \\
\hline MboII & GAAGA & 5 & three_prime & 6 & $105,169,252,642,648,963$ \\
\hline BbvI & GCAGC & 5 & five_prime & 9 & $11,72,413,566,735,828,897,1101,1187$ \\
\hline TseI & GCWGC & 5 & five_prime & 9 & $24,85,401,554,748,841,910,1114,1175$ \\
\hline
\end{tabular}

Table 10: Restriction digestion of protease gene from XM_024823318.1 Aspergillus novofumigatus

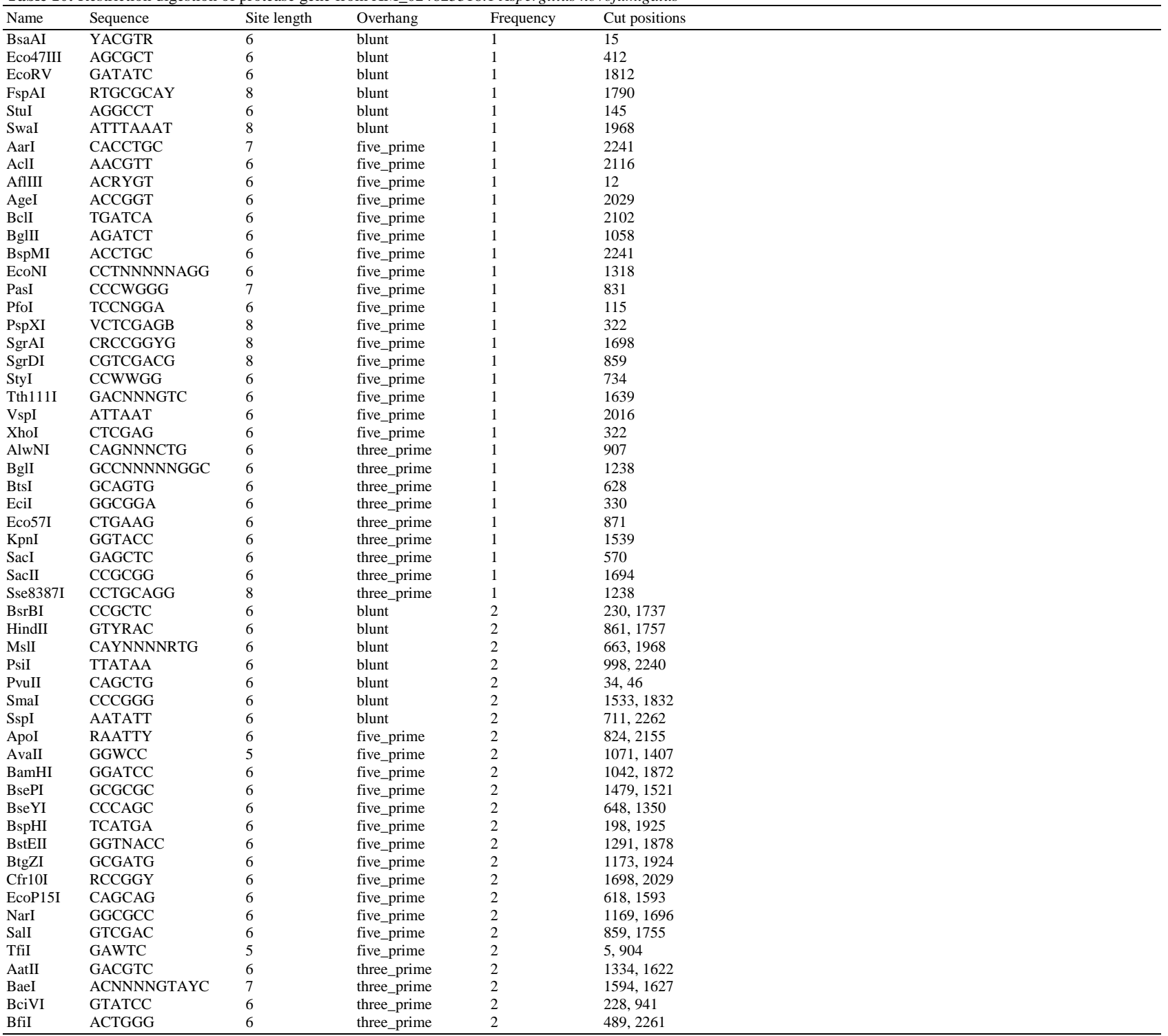


Samia Abd Allah Abdal-Aziz and Safaa M. Ali / OnLine Journal of Biological Sciences 2021, 21 (1): 69.119 DOI: 10.3844/ojbsci.2021.69.119

\begin{tabular}{|c|c|c|c|c|c|}
\hline BplI & GAGNNNNNCTC & 6 & three_prime & 2 & 305,337 \\
\hline BsaXI & ACNNNNNCTCC & 6 & three_prime & 2 & 1045,1075 \\
\hline DrdI & GACNNNNNNGTC & 6 & three_prime & 2 & 1273,1642 \\
\hline NspI & RCATGY & 6 & three_prime & 2 & 1665,1987 \\
\hline SphI & GCATGC & 6 & three_prime & 2 & 1665,1987 \\
\hline AccI & GTMKAC & 6 & five_prime & 3 & $860,1130,1756$ \\
\hline CfrI & YGGCCR & 6 & five_prime & 3 & $943,1625,1709$ \\
\hline SmlI & CTYRAG & 6 & five_prime & 3 & $322,616,869$ \\
\hline BseMII & CTCAG & 5 & three_prime & 3 & $320,370,398$ \\
\hline BseSI & GKGCMC & 6 & three_prime & 3 & $838,1684,2058$ \\
\hline GsuI & CTGGAG & 6 & three_prime & 3 & $1102,1972,1979$ \\
\hline MmeI & TCCRAC & 6 & three_prime & 3 & $365,914,1895$ \\
\hline PstI & CTGCAG & 6 & three_prime & 3 & $33,45,1238$ \\
\hline AvaI & CYCGRG & 6 & five_prime & 4 & $322,1315,1531,1830$ \\
\hline Esp3I & CGTCTC & 6 & five_prime & 4 & $121,239,1248,1997$ \\
\hline PleI & GAGTC & 5 & five_prime & 4 & $333,565,1261,1887$ \\
\hline Tsp45I & GTSAC & 5 & five_prime & 4 & $1291,1327,1434,1878$ \\
\hline BcgI & CGANNNNNNTGC & 6 & three_prime & 4 & $76,110,262,296$ \\
\hline BsrDI & GCAATG & 6 & three_prime & 4 & $379,467,658,747$ \\
\hline TspGWI & ACGGA & 5 & three_prime & 4 & $136,246,1055,1236$ \\
\hline Eco31I & GGTCTC & 6 & five_prime & 5 & $175,1501,2078,2099,2155$ \\
\hline HgaI & GACGC & 5 & five_prime & 5 & $1116,1230,1263,1613,1677$ \\
\hline XhoII & RGATCY & 6 & five_prime & 5 & $762,789,1042,1058,1872$ \\
\hline BsrI & ACTGG & 5 & three_prime & 5 & $495,1011,1119,1206,2256$ \\
\hline HaeII & RGCGCY & 6 & three_prime & 5 & $414,869,1172,1203,1699$ \\
\hline HphI & GGTGA & 5 & three_prime & 5 & 1303, 1339, 1362, 1890, 2097 \\
\hline MboII & GAAGA & 5 & three_prime & 5 & $274,795,1326,1435,1641$ \\
\hline TspRI & CASTG & 5 & three_prime & 5 & $506,635,1011,1126,1213$ \\
\hline AgsI & TTSAA & 5 & three_prime & 6 & $580,922,1653,2022,2206,2266$ \\
\hline BdaI & TGANNNNNNTCA & 6 & three_prime & 6 & $1707,1741,1993,2027,2158,2192$ \\
\hline HaeIV & GAYNNNNNRTC & 6 & three_prime & 6 & $235,269,287,321,959,993$ \\
\hline NmeAIII & GCCGAG & 6 & three_prime & 6 & $1197,1208,1239,1379,1599,1834$ \\
\hline SduI & GDGCHC & 6 & three_prime & 6 & $56,570,838,1151,1684,2058$ \\
\hline AcyI & GRCGYC & 6 & five_prime & 7 & $1127,1169,1241,1274,1331,1619,1696$ \\
\hline EcoRII & CCWGG & 5 & five_prime & 7 & $20,115,290,830,911,1720,2213$ \\
\hline SfaNI & GCATC & 5 & five_prime & 7 & $359,368,542,761,1020,1267,1800$ \\
\hline BbvI & GCAGC & 5 & five_prime & 8 & $15,27,43,55,590,632,1577,1845$ \\
\hline BccI & CCATC & 5 & five_prime & 8 & $204,853,964,1162,1195,1636,1720,1862$ \\
\hline FauI & CCCGC & 5 & five_prime & 8 & $235,608,693,1082,1098,1375,1420,1450$ \\
\hline BsmAI & GTCTC & 5 & five_prime & 10 & $121,175,239,289,1248,1501,1997,2078,2099,2155$ \\
\hline FokI & GGATG & 5 & five_prime & 12 & $390,784,827,832,943,1233,1245,1432,1699,1778,1880,1989$ \\
\hline Hpy99I & CGWCG & 5 & three_prime & 12 & $98,262,862,865,1099,1252,1255,1258,1279,1624,1645,1671$ \\
\hline TauI & GCSGC & 5 & three_prime & 15 & $609,624,948,1103,1127,1169,1205,1457,1583,1586,1627,1693,1696,1711,1740$ \\
\hline Hin4I & GAYNNNNNVTC & 6 & three_prime & 16 & $130,153,162,185,214,236,246,268,288,320,322,354,960,992,1050,1082$ \\
\hline
\end{tabular}

Table 11: Restriction digestion of protease gene from XM_002374250.1 Aspergillus flavus

\begin{tabular}{|c|c|c|c|c|c|}
\hline Name & Sequence & Site length & Overhang & Frequency & Cut positions \\
\hline BsaAI & YACGTR & 6 & blunt & 1 & 477 \\
\hline EcoRV & GATATC & 6 & blunt & 1 & 990 \\
\hline StuI & AGGCCT & 6 & blunt & 1 & 535 \\
\hline $\mathrm{XmnI}$ & GAANNNNTTC & 6 & blunt & 1 & 811 \\
\hline AccI & GTMKAC & 6 & five_prime & 1 & 500 \\
\hline BglII & AGATCT & 6 & five_prime & 1 & 347 \\
\hline BspMI & ACCTGC & 6 & five_prime & 1 & 1193 \\
\hline EcoP15I & CAGCAG & 6 & five_prime & 1 & 451 \\
\hline $\mathrm{HgaI}$ & GACGC & 5 & five_prime & 1 & 187 \\
\hline HindIII & AAGCTT & 6 & five_prime & 1 & 94 \\
\hline NarI & GGCGCC & 6 & five_prime & 1 & 206 \\
\hline PasI & CCCWGGG & 7 & five_prime & 1 & 389 \\
\hline PfoI & TCCNGGA & 6 & five_prime & 1 & 99 \\
\hline SalI & GTCGAC & 6 & five_prime & 1 & 499 \\
\hline SmlI & CTYRAG & 6 & five_prime & 1 & 1093 \\
\hline SpeI & ACTAGT & 6 & five_prime & 1 & 448 \\
\hline TatI & WGTACW & 6 & five_prime & 1 & 107 \\
\hline Tth111I & GACNNNGTC & 6 & five_prime & 1 & 1156 \\
\hline BseMII & CTCAG & 5 & three_prime & 1 & 80 \\
\hline BsgI & GTGCAG & 6 & three_prime & 1 & 88 \\
\hline BsmI & GAATGC & 6 & three_prime & 1 & 811 \\
\hline BsrDI & GCAATG & 6 & three_prime & 1 & 599 \\
\hline Eco57MI & CTGRAG & 6 & three_prime & 1 & 214 \\
\hline GsuI & CTGGAG & 6 & three_prime & 1 & 214 \\
\hline HaeII & RGCGCY & 6 & three_prime & 1 & 209 \\
\hline KpnI & GGTACC & 6 & three_prime & 1 & 1043 \\
\hline MmeI & TCCRAC & 6 & three_prime & 1 & 971 \\
\hline
\end{tabular}


Samia Abd Allah Abdal-Aziz and Safaa M. Ali / OnLine Journal of Biological Sciences 2021, 21 (1): 69.119 DOI: 10.3844/ojbsci.2021.69.119

\begin{tabular}{|c|c|c|c|c|c|}
\hline NspI & RCATGY & 6 & three_prime & 1 & 562 \\
\hline SacII & CCGCGG & 6 & three_prime & 1 & 50 \\
\hline TspGWI & ACGGA & 5 & three_prime & 1 & 672 \\
\hline HindII & GTYRAC & 6 & blunt & 2 & 501,966 \\
\hline NaeI & GCCGGC & 6 & blunt & 2 & 456,867 \\
\hline AvalI & GGWCC & 5 & five_prime & 2 & 51,342 \\
\hline FokI & GGATG & 5 & five_prime & 2 & 625,1177 \\
\hline SapI & GCTCTTC & 7 & five_prime & 2 & 916,1017 \\
\hline Tsp45I & GTSAC & 5 & five_prime & 2 & 114,1119 \\
\hline AatII & GACGTC & 6 & three_prime & 2 & 971,1157 \\
\hline BglI & GCCNNNNNGGC & 6 & three_prime & 2 & 532,631 \\
\hline FalI & AAGNNNNNCTT & 6 & three_prime & 2 & 977,1009 \\
\hline TaqII & GACCGA & 6 & three_prime & 2 & 636,662 \\
\hline TsoI & TARCCA & 6 & three_prime & 2 & 764,1039 \\
\hline TspRI & CASTG & 5 & three_prime & 2 & 216,909 \\
\hline AcyI & GRCGYC & 6 & five_prime & 3 & $206,968,1154$ \\
\hline StyI & CCWWGG & 6 & five_prime & 3 & $576,1046,1148$ \\
\hline TfiI & GAWTC & 5 & five_prime & 3 & $147,306,667$ \\
\hline AgsI & TTSAA & 5 & three_prime & 3 & $123,702,792$ \\
\hline BseSI & GKGCMC & 6 & three_prime & 3 & $63,387,466$ \\
\hline BsrI & ACTGG & 5 & three_prime & 3 & $216,709,938$ \\
\hline HphI & GGTGA & 5 & three_prime & 3 & $172,229,676$ \\
\hline Cfr10I & RCCGGY & 6 & five_prime & 4 & $454,547,844,865$ \\
\hline $\mathrm{BcgI}$ & CGANNNNNNTGC & 6 & three_prime & 4 & $307,341,433,467$ \\
\hline Нpy99I & CGWCG & 5 & three_prime & 4 & $203,289,337,1159$ \\
\hline SduI & GDGCHC & 6 & three_prime & 4 & $63,387,430,466$ \\
\hline TauI & GCSGC & 5 & three_prime & 4 & $88,268,524,913$ \\
\hline BccI & CCATC & 5 & five_prime & 5 & $16,355,907,1039,1099$ \\
\hline EcoRII & CCWGG & 5 & five_prime & 5 & $99,163,388,659,1000$ \\
\hline BseRI & GAGGAG & 6 & three_prime & 5 & $16,19,316,352,523$ \\
\hline SfaNI & GCATC & 5 & five_prime & 6 & $279,647,786,852,885,1139$ \\
\hline MboII & GAAGA & 5 & three_prime & 6 & $214,331,390,933,963,1004$ \\
\hline BbvI & GCAGC & 5 & five_prime & 9 & $413,531,686,696,735,828,1074,1101,1187$ \\
\hline TseI & GCWGC & 5 & five_prime & 9 & $401,544,674,709,748,841,1087,1114,1175$ \\
\hline
\end{tabular}

Table 12: Restriction digestion of protease gene from AF324246.1 Aspergillus flavus

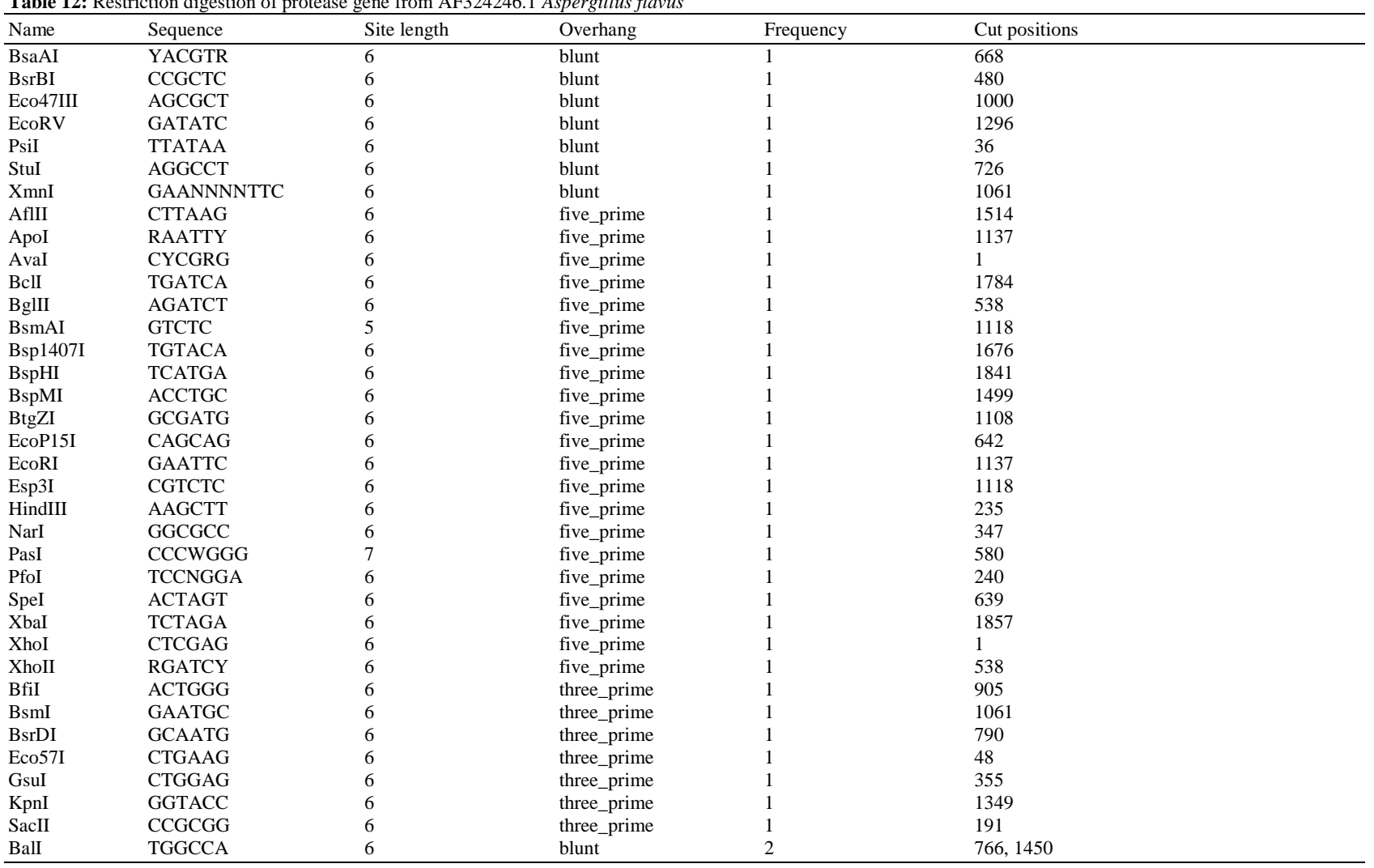


Samia Abd Allah Abdal-Aziz and Safaa M. Ali / OnLine Journal of Biological Sciences 2021, 21 (1): 69.119 DOI: 10.3844/ojbsci.2021.69.119

\begin{tabular}{|c|c|c|c|c|c|}
\hline NaeI & GCCGGC & 6 & blunt & 2 & 647,1173 \\
\hline AvaII & GGWCC & 5 & five_prime & 2 & 192,533 \\
\hline HgaI & GACGC & 5 & five_prime & 2 & 328,1842 \\
\hline NcoI & CCATGG & 6 & five_prime & 2 & 767,1352 \\
\hline SalI & GTCGAC & 6 & five_prime & 2 & 690,1818 \\
\hline TatI & WGTACW & 6 & five_prime & 2 & 248,1676 \\
\hline Tsp45I & GTSAC & 5 & five_prime & 2 & 255,1425 \\
\hline Tth111I & GACNNNGTC & 6 & five_prime & 2 & 1462,1871 \\
\hline AatII & GACGTC & 6 & three prime & 2 & 1277,1463 \\
\hline ArsI & GACNNNNNNTTYG & 7 & three_prime & 2 & 1812,1844 \\
\hline $\mathrm{BcgI}$ & CGANNNNNNTGC & 6 & three_prime & 2 & 624,658 \\
\hline BdaI & TGANNNNNNTCA & 6 & three_prime & 2 & 1821,1855 \\
\hline $\mathrm{BglI}$ & GCCNNNNNGGC & 6 & three_prime & 2 & 723,822 \\
\hline BseMII & CTCAG & 5 & three_prime & 2 & 221,1787 \\
\hline BsgI & GTGCAG & 6 & three_prime & 2 & 229,1792 \\
\hline Eco57MI & CTGRAG & 6 & three_prime & 2 & 48,355 \\
\hline HaeII & RGCGCY & 6 & three_prime & 2 & 350,1002 \\
\hline MmeI & TCCRAC & 6 & three_prime & 2 & 124,1277 \\
\hline TsoI & TARCCA & 6 & three_prime & 2 & 1014,1345 \\
\hline TspGWI & ACGGA & 5 & three_prime & 2 & 863,1615 \\
\hline TspRI & CASTG & 5 & three_prime & 2 & 357,1215 \\
\hline HindII & GTYRAC & 6 & blunt & 3 & $692,1272,1820$ \\
\hline AccI & GTMKAC & 6 & five_prime & 3 & $691,1685,1819$ \\
\hline AcyI & GRCGYC & 6 & five_prime & 3 & $347,1274,1460$ \\
\hline CfrI & YGGCCR & 6 & five_prime & 3 & $764,1173,1448$ \\
\hline FauI & CCCGC & 5 & five_prime & 3 & $195,226,1425$ \\
\hline SapI & GCTCTTC & 7 & five_prime & 3 & $1222,1323,1576$ \\
\hline StyI & CCWWGG & 6 & five_prime & 3 & $767,1352,1454$ \\
\hline BseSI & GKGCMC & 6 & three_prime & 3 & $204,578,657$ \\
\hline Cfr10I & RCCGGY & 6 & five_prime & 4 & $645,738,1094,1171$ \\
\hline BsrI & ACTGG & 5 & three_prime & 4 & $357,900,1244,1559$ \\
\hline FalI & AAGNNNNNCTT & 6 & three_prime & 4 & $145,177,1283,1315$ \\
\hline SduI & GDGCHC & 6 & three_prime & 4 & $204,578,621,657$ \\
\hline TauI & GCSGC & 5 & three_prime & 4 & $229,409,715,1219$ \\
\hline FokI & GGATG & 5 & five_prime & 5 & $36,47,461,816,1483$ \\
\hline SmlI & CTYRAG & 6 & five_prime & 5 & $1,104,1399,1514,1647$ \\
\hline Hpy99I & CGWCG & 5 & three_prime & 6 & $344,430,528,1465,1535,1751$ \\
\hline EcoRII & CCWGG & 5 & five_prime & 7 & $240,304,511,579,850,1306,1521$ \\
\hline TfiI & GAWTC & 5 & five_prime & 7 & $288,447,858,1539,1593,1669,1838$ \\
\hline BccI & CCATC & 5 & five_prime & 8 & $68,80,157,546,1130,1213,1345,1405$ \\
\hline SfaNI & GCATC & 5 & five_prime & 8 & $58,129,420,838,1036,1158,1191,1445$ \\
\hline BbvI & GCAGC & 5 & five_prime & 9 & $604,722,877,887,926,1078,1380,1407,1493$ \\
\hline TseI & GCWGC & 5 & five_prime & 9 & $592,735,865,900,939,1091,1393,1420,1481$ \\
\hline MboII & GAAGA & 5 & three_prime & 9 & $124,355,472,581,968,1239,1269,1310,1593$ \\
\hline
\end{tabular}

Table 13: Restriction digestion of protease gene from GQ925367.1 Aspergillus clavatus

\begin{tabular}{|c|c|c|c|c|c|}
\hline Name & Sequence & Site length & Overhang & Frequency & Cut positions \\
\hline BsrBI & CCGCTC & 6 & blunt & 1 & 889 \\
\hline HpaI & GTTAAC & 6 & blunt & 1 & 501 \\
\hline NaeI & GCCGGC & 6 & blunt & 1 & 47 \\
\hline PvuII & CAGCTG & 6 & blunt & 1 & 1140 \\
\hline StuI & AGGCCT & 6 & blunt & 1 & 535 \\
\hline $\mathrm{XmnI}$ & GAANNNNTTC & 6 & blunt & 1 & 244 \\
\hline AclI & AACGTT & 6 & five_prime & 1 & 497 \\
\hline AcyI & GRCGYC & 6 & five_prime & 1 & 1154 \\
\hline AflIII & ACRYGT & 6 & five_prime & 1 & 863 \\
\hline BglII & AGATCT & 6 & five_prime & 1 & 347 \\
\hline BstEII & GGTNACC & 6 & five_prime & 1 & 553 \\
\hline DraII & RGGNCCY & 6 & five_prime & 1 & 278 \\
\hline Eco31I & GGTCTC & 6 & five_prime & 1 & 400 \\
\hline FauI & CCCGC & 5 & five_prime & 1 & 940 \\
\hline MluI & ACGCGT & 6 & five_prime & 1 & 863 \\
\hline $\mathrm{NcoI}$ & CCATGG & 6 & five_prime & 1 & 1046 \\
\hline NheI & GCTAGC & 6 & five_prime & 1 & 424 \\
\hline PpuMI & RGGWCCY & 7 & five_prime & 1 & 278 \\
\hline SmlI & CTYRAG & 6 & five_prime & 1 & 1093 \\
\hline TatI & WGTACW & 6 & five_prime & 1 & 107 \\
\hline XhoII & RGATCY & 6 & five_prime & 1 & 347 \\
\hline BfiI & ACTGGG & 6 & three_prime & 1 & 714 \\
\hline BseSI & GKGCMC & 6 & three_prime & 1 & 387 \\
\hline
\end{tabular}


Samia Abd Allah Abdal-Aziz and Safaa M. Ali / OnLine Journal of Biological Sciences 2021, 21 (1): 69.119 DOI: 10.3844/ojbsci.2021.69.119

\begin{tabular}{|c|c|c|c|c|c|}
\hline BsrDI & GCAATG & 6 & three_prime & 1 & 933 \\
\hline BstXI & CCANNNNNNTGG & 6 & three_prime & 1 & 1066 \\
\hline BtsI & GCAGTG & 6 & three_prime & 1 & 1016 \\
\hline DrdI & GACNNNNNNGTC & 6 & three_prime & 1 & 375 \\
\hline EciI & GGCGGA & 6 & three_prime & 1 & 987 \\
\hline HaeII & RGCGCY & 6 & three_prime & 1 & 455 \\
\hline NmeAIII & GCCGAG & 6 & three_prime & 1 & 377 \\
\hline TspGWI & ACGGA & 5 & three_prime & 1 & 593 \\
\hline Ball & TGGCCA & 6 & blunt & 2 & 625,983 \\
\hline AccI & GTMKAC & 6 & five_prime & 2 & 860,1079 \\
\hline AvalI & GGWCC & 5 & five_prime & 2 & 278,1108 \\
\hline BseYI & CCCAGC & 6 & five_prime & 2 & 274,1124 \\
\hline BsmAI & GTCTC & 5 & five_prime & 2 & 400,1107 \\
\hline CfrI & YGGCCR & 6 & five_prime & 2 & 623,981 \\
\hline EcoP15I & CAGCAG & 6 & five_prime & 2 & 199,1084 \\
\hline FokI & GGATG & 5 & five_prime & 2 & 82,156 \\
\hline $\mathrm{BcgI}$ & CGANNNNNNTGC & 6 & three_prime & 2 & 208,242 \\
\hline BglI & GCCNNNNNGGC & 6 & three_prime & 2 & 212,740 \\
\hline BplI & GAGNNNNNCTC & 6 & three_prime & 2 & 1087,1119 \\
\hline BsaXI & ACNNNNNCTCC & 6 & three_prime & 2 & 1018,1048 \\
\hline BsrI & ACTGG & 5 & three_prime & 2 & 671,709 \\
\hline HaeIV & GAYNNNNNRTC & 6 & three_prime & 2 & 978,1012 \\
\hline Hin4I & GAYNNNNNVTC & 6 & three_prime & 2 & 979,1011 \\
\hline HphI & GGTGA & 5 & three_prime & 2 & 70,565 \\
\hline PpiI & GAACNNNNNCTC & 7 & three_prime & 2 & 229,261 \\
\hline PsrI & GAACNNNNNNTAC & 7 & three_prime & 2 & 660,692 \\
\hline SduI & GDGCHC & 6 & three_prime & 2 & 39,387 \\
\hline TaqII & GACCGA & 6 & three_prime & 2 & 672,698 \\
\hline TsoI & TARCCA & 6 & three_prime & 2 & 517,1039 \\
\hline TspRI & CASTG & 5 & three_prime & 2 & 138,1023 \\
\hline TstI & CACNNNNNNTCC & 6 & three_prime & 2 & 70,102 \\
\hline HindII & GTYRAC & 6 & blunt & 3 & $483,501,861$ \\
\hline PleI & GAGTC & 5 & five_prime & 3 & $159,478,770$ \\
\hline Tsp45I & GTSAC & 5 & five_prime & 3 & $553,574,1119$ \\
\hline Eco57MI & CTGRAG & 6 & three_prime & 3 & $214,996,1120$ \\
\hline GsuI & CTGGAG & 6 & three_prime & 3 & $214,996,1120$ \\
\hline KpnI & GGTACC & 6 & three_prime & 3 & $357,599,1043$ \\
\hline BccI & CCATC & 5 & five_prime & 4 & $16,304,688,1039$ \\
\hline Cfr10I & RCCGGY & 6 & five_prime & 4 & $45,454,592,844$ \\
\hline HgaI & GACGC & 5 & five_prime & 4 & $187,854,871,1143$ \\
\hline AgsI & TTSAA & 5 & three_prime & 4 & $123,249,702,792$ \\
\hline AlfI & GCANNNNNNTGC & 6 & three_prime & 4 & $6,40,364,398$ \\
\hline Hpy99I & CGWCG & 5 & three_prime & 4 & $203,481,865,910$ \\
\hline MmeI & TCCRAC & 6 & three_prime & 4 & $113,197,313,971$ \\
\hline SfaNI & GCATC & 5 & five_prime & 5 & $178,242,351,413,500$ \\
\hline StyI & CCWWGG & 6 & five_prime & 5 & $30,388,530,570,1046$ \\
\hline MboII & GAAGA & 5 & three_prime & 6 & $77,252,349,352,648,963$ \\
\hline EcoRII & CCWGG & 5 & five_prime & 7 & $99,163,191,515,978,983,1000$ \\
\hline TauI & GCSGC & 5 & three_prime & 7 & $145,206,218,268,524,608,844$ \\
\hline BbvI & GCAGC & 5 & five_prime & 8 & $45,61,413,696,735,887,1101,1191$ \\
\hline TseI & GCWGC & 5 & five_prime & 8 & $49,58,401,709,748,875,1114,1204$ \\
\hline
\end{tabular}

Table 14: Restriction digestion of protease gene from XM_001272037.1 Aspergillus clavatus

\begin{tabular}{|c|c|c|c|c|c|}
\hline Name & Sequence & Site length & Overhang & Frequency & Cut positions \\
\hline BsrBI & CCGCTC & 6 & blunt & 1 & 889 \\
\hline BtrI & CACGTC & 6 & blunt & 1 & 561 \\
\hline EcoRV & GATATC & 6 & blunt & 1 & 990 \\
\hline NaeI & GCCGGC & 6 & blunt & 1 & 47 \\
\hline PvuII & CAGCTG & 6 & blunt & 1 & 1140 \\
\hline StuI & AGGCCT & 6 & blunt & 1 & 535 \\
\hline AclI & AACGTT & 6 & five_prime & 1 & 497 \\
\hline AcyI & GRCGYC & 6 & five_prime & 1 & 1154 \\
\hline AgeI & ACCGGT & 6 & five_prime & 1 & 670 \\
\hline AvaII & GGWCC & 5 & five_prime & 1 & 1108 \\
\hline BglII & AGATCT & 6 & five_prime & 1 & 347 \\
\hline BstEII & GGTNACC & 6 & five_prime & 1 & 553 \\
\hline Eco31I & GGTCTC & 6 & five_prime & 1 & 400 \\
\hline NcoI & CCATGG & 6 & five_prime & 1 & 1046 \\
\hline NheI & GCTAGC & 6 & five_prime & 1 & 424 \\
\hline SalI & GTCGAC & 6 & five_prime & 1 & 859 \\
\hline SgrAI & CRCCGGYG & 8 & five_prime & 1 & 454 \\
\hline SmlI & CTYRAG & 6 & five_prime & 1 & 1093 \\
\hline XhoII & RGATCY & 6 & five_prime & 1 & 347 \\
\hline BfiI & ACTGGG & 6 & three_prime & 1 & 714 \\
\hline BseMII & CTCAG & 5 & three_prime & 1 & 1138 \\
\hline
\end{tabular}


Samia Abd Allah Abdal-Aziz and Safaa M. Ali / OnLine Journal of Biological Sciences 2021, 21 (1): 69.119 DOI: 10.3844/ojbsci.2021.69.119

\begin{tabular}{|c|c|c|c|c|c|}
\hline BseRI & GAGGAG & 6 & three_prime & 1 & 16 \\
\hline BseSI & GKGCMC & 6 & three_prime & 1 & 387 \\
\hline BsrDI & GCAATG & 6 & three_prime & 1 & 933 \\
\hline BsrI & ACTGG & 5 & three_prime & 1 & 709 \\
\hline BstXI & CCANNNNNNTGG & 6 & three_prime & 1 & 1066 \\
\hline BtsI & GCAGTG & 6 & three_prime & 1 & 1016 \\
\hline DrdI & GACNNNNNNGTC & 6 & three_prime & 1 & 375 \\
\hline EciI & GGCGGA & 6 & three_prime & 1 & 987 \\
\hline HaeII & RGCGCY & 6 & three_prime & 1 & 455 \\
\hline NmeAIII & GCCGAG & 6 & three_prime & 1 & 377 \\
\hline TspGWI & ACGGA & 5 & three_prime & 1 & 593 \\
\hline TspRI & CASTG & 5 & three_prime & 1 & 1023 \\
\hline BalI & TGGCCA & 6 & blunt & 2 & 983,1144 \\
\hline AccI & GTMKAC & 6 & five_prime & 2 & 860,1079 \\
\hline FauI & CCCGC & 5 & five_prime & 2 & 208,940 \\
\hline AlfI & GCANNNNNNTGC & 6 & three_prime & 2 & 364,398 \\
\hline $\mathrm{BcgI}$ & CGANNNNNNTGC & 6 & three_prime & 2 & 307,341 \\
\hline BglI & GCCNNNNNGGC & 6 & three_prime & 2 & 212,740 \\
\hline BplI & GAGNNNNNCTC & 6 & three_prime & 2 & 1087,1119 \\
\hline BsaXI & ACNNNNNCTCC & 6 & three_prime & 2 & 1018,1048 \\
\hline Eco57MI & CTGRAG & 6 & three_prime & 2 & 214,1120 \\
\hline GsuI & CTGGAG & 6 & three_prime & 2 & 214,1120 \\
\hline HaeIV & GAYNNNNNRTC & 6 & three_prime & 2 & 978,1012 \\
\hline HphI & GGTGA & 5 & three_prime & 2 & 70,565 \\
\hline KpnI & GGTACC & 6 & three_prime & 2 & 357,599 \\
\hline PpiI & GAACNNNNNCTC & 7 & three_prime & 2 & 229,261 \\
\hline PsrI & GAACNNNNNNTAC & 7 & three_prime & 2 & 660,692 \\
\hline SduI & GDGCHC & 6 & three_prime & 2 & 39,387 \\
\hline TaqII & GACCGA & 6 & three_prime & 2 & 672,698 \\
\hline TsoI & TARCCA & 6 & three_prime & 2 & 613,1039 \\
\hline TstI & CACNNNNNNTCC & 6 & three_prime & 2 & 70,102 \\
\hline HindII & GTYRAC & 6 & blunt & 3 & $483,501,861$ \\
\hline BsmAI & GTCTC & 5 & five_prime & 3 & $158,400,1107$ \\
\hline CfrI & YGGCCR & 6 & five_prime & 3 & $65,981,1142$ \\
\hline EcoP15I & CAGCAG & 6 & five_prime & 3 & $12,199,1084$ \\
\hline FokI & GGATG & 5 & five_prime & 3 & $82,156,706$ \\
\hline HgaI & GACGC & 5 & five_prime & 3 & $187,871,1143$ \\
\hline Tsp45I & GTSAC & 5 & five_prime & 3 & $553,574,1119$ \\
\hline MmeI & TCCRAC & 6 & three_prime & 3 & $113,197,971$ \\
\hline $\mathrm{BccI}$ & CCATC & 5 & five_prime & 4 & $16,304,688,1039$ \\
\hline PleI & GAGTC & 5 & five_prime & 4 & $159,478,770,1116$ \\
\hline AgsI & TTSAA & 5 & three_prime & 4 & $123,249,702,792$ \\
\hline Hin4I & GAYNNNNNVTC & 6 & three_prime & 4 & $138,170,979,1011$ \\
\hline Cfr10I & RCCGGY & 6 & five_prime & 5 & $45,454,592,670,844$ \\
\hline SfaNI & GCATC & 5 & five_prime & 5 & $178,242,351,413,500$ \\
\hline StyI & CCWWGG & 6 & five_prime & 5 & $30,388,530,570,1046$ \\
\hline Нpy99I & CGWCG & 5 & three_prime & 5 & $203,481,565,865,910$ \\
\hline MboII & GAAGA & 5 & three_prime & 5 & $77,331,352,648,963$ \\
\hline TauI & GCSGC & 5 & three_prime & 7 & $145,206,268,524,608,737,844$ \\
\hline EcoRII & CCWGG & 5 & five_prime & 8 & $67,99,163,191,515,978,983,1000$ \\
\hline BbvI & GCAGC & 5 & five_prime & 9 & $29,45,61,413,696,735,887,1101,1191$ \\
\hline TseI & GCWGC & 5 & five_prime & 9 & $42,49,58,401,709,748,875,1114,1204$ \\
\hline
\end{tabular}

Table 15: Restriction digestion of protease gene from XM_032052352.1 Aspergillus pseudotamarii

\begin{tabular}{|c|c|c|c|c|c|}
\hline Name & Sequence & Site length & Overhang & Frequency & Cut positions \\
\hline$\overline{\text { BsaAI }}$ & YACGTR & 6 & blunt & 1 & 1085 \\
\hline BsrBI & CCGCTC & 6 & blunt & 1 & 2094 \\
\hline HpaI & GTTAAC & 6 & blunt & 1 & 1790 \\
\hline MslI & CAYNNNNRTG & 6 & blunt & 1 & 1867 \\
\hline OliI & CACNNNNGTG & 6 & blunt & 1 & 1867 \\
\hline PsiI & TTATAA & 6 & blunt & 1 & 503 \\
\hline SmaI & CCCGGG & 6 & blunt & 1 & 2236 \\
\hline StuI & AGGCCT & 6 & blunt & 1 & 1143 \\
\hline $\mathrm{XmnI}$ & GAANNNNTTC & 6 & blunt & 1 & 1013 \\
\hline AflII & CTTAAG & 6 & five_prime & 1 & 1816 \\
\hline Bpu10I & CCTNAGC & 6 & five_prime & 1 & 147 \\
\hline BspHI & TCATGA & 6 & five_prime & 1 & 2142 \\
\hline BspMI & ACCTGC & 6 & five_prime & 1 & 1801 \\
\hline BtgZI & GCGATG & 6 & five_prime & 1 & 4 \\
\hline NarI & GGCGCC & 6 & five_prime & 1 & 814 \\
\hline PasI & CCCWGGG & 7 & five_prime & 1 & 997 \\
\hline PfoI & TCCNGGA & 6 & five_prime & 1 & 707 \\
\hline SexAI & ACCWGGT & 7 & five_prime & 1 & 1823 \\
\hline XhoII & RGATCY & 6 & five_prime & 1 & 913 \\
\hline AatII & GACGTC & 6 & three_prime & 1 & 1765 \\
\hline
\end{tabular}


Samia Abd Allah Abdal-Aziz and Safaa M. Ali / OnLine Journal of Biological Sciences 2021, 21 (1): 69.119 DOI: 10.3844/ojbsci.2021.69.119

\begin{tabular}{|c|c|c|c|c|c|}
\hline BfiI & ACTGGG & 6 & three_prime & 1 & 1322 \\
\hline EciI & GGCGGA & 6 & three_prime & 1 & 1595 \\
\hline Eco57I & CTGAAG & 6 & three_prime & 1 & 288 \\
\hline HaeII & RGCGCY & 6 & three_prime & 1 & 817 \\
\hline NmeAIII & GCCGAG & 6 & three_prime & 1 & 722 \\
\hline PvuI & CGATCG & 6 & three_prime & 1 & 2054 \\
\hline SphI & GCATGC & 6 & three_prime & 1 & 436 \\
\hline BsaBI & GATNNNNATC & 6 & blunt & 2 & 2084,2203 \\
\hline NaeI & GCCGGC & 6 & blunt & 2 & 1064,1475 \\
\hline AccI & GTMKAC & 6 & five_prime & 2 & 1108,1573 \\
\hline AcyI & GRCGYC & 6 & five_prime & 2 & 814,1762 \\
\hline BsmAI & GTCTC & 5 & five_prime & 2 & 193,675 \\
\hline Eco31I & GGTCTC & 6 & five_prime & 2 & 193,675 \\
\hline EcoP15I & CAGCAG & 6 & five_prime & 2 & 1125,1692 \\
\hline HgaI & GACGC & 5 & five_prime & 2 & 269,795 \\
\hline NcoI & CCATGG & 6 & five_prime & 2 & 1184,1654 \\
\hline SalI & GTCGAC & 6 & five_prime & 2 & 1107,1572 \\
\hline XhoI & CTCGAG & 6 & five_prime & 2 & 209,468 \\
\hline AlfI & GCANNNNNNTGC & 6 & three_prime & 2 & 574,608 \\
\hline AloI & GAACNNNNNNTCC & 7 & three_prime & 2 & 242,274 \\
\hline BdaI & TGANNNNNNTCA & 6 & three_prime & 2 & 257,291 \\
\hline BglI & GCCNNNNNGGC & 6 & three_prime & 2 & 1140,1239 \\
\hline BsaXI & ACNNNNNCTCC & 6 & three_prime & 2 & 242,272 \\
\hline BseSI & GKGCMC & 6 & three_prime & 2 & 671,1074 \\
\hline BsgI & GTGCAG & 6 & three_prime & 2 & 696,2093 \\
\hline BsmI & GAATGC & 6 & three_prime & 2 & 995,1421 \\
\hline Eco57MI & CTGRAG & 6 & three_prime & 2 & 288,822 \\
\hline KpnI & GGTACC & 6 & three_prime & 2 & 134,1651 \\
\hline NspI & RCATGY & 6 & three_prime & 2 & 436,516 \\
\hline PpiI & GAACNNNNNCTC & 7 & three_prime & 2 & 242,274 \\
\hline SacII & CCGCGG & 6 & three_prime & 2 & 28,658 \\
\hline TaqII & GACCGA & 6 & three_prime & 2 & 1244,1270 \\
\hline BalI & TGGCCA & 6 & blunt & 3 & $1659,1752,2123$ \\
\hline EcoRV & GATATC & 6 & blunt & 3 & $54,1598,2326$ \\
\hline HindII & GTYRAC & 6 & blunt & 3 & $1109,1574,1790$ \\
\hline DraII & RGGNCCY & 6 & five_prime & 3 & $36,1001,2188$ \\
\hline HindIII & AAGCTT & 6 & five_prime & 3 & $597,702,2300$ \\
\hline Tth111I & GACNNNGTC & 6 & five_prime & 3 & $1764,2173,2186$ \\
\hline BsrDI & GCAATG & 6 & three_prime & 3 & $382,427,1207$ \\
\hline MmeI & TCCRAC & 6 & three_prime & 3 & $591,1579,1923$ \\
\hline TspDTI & ATGAA & 5 & three_prime & 3 & $2022,2096,2257$ \\
\hline AvalI & GGWCC & 5 & five_prime & 4 & $36,659,950,2188$ \\
\hline Cfr10I & RCCGGY & 6 & five_prime & 4 & $819,1062,1452,1473$ \\
\hline StyI & CCWWGG & 6 & five_prime & 4 & $1184,1390,1654,1756$ \\
\hline Tsp45I & GTSAC & 5 & five_prime & 4 & $379,722,1727,2175$ \\
\hline BseMII & CTCAG & 5 & three_prime & 4 & $93,138,357,1724$ \\
\hline BsrI & ACTGG & 5 & three_prime & 4 & $1057,1317,1546,2311$ \\
\hline FalI & AAGNNNNNCTT & 6 & three_prime & 4 & $612,644,2177,2209$ \\
\hline Hin4I & GAYNNNNNVTC & 6 & three_prime & 4 & $203,235,565,597$ \\
\hline TspGWI & ACGGA & 5 & three_prime & 4 & $273,1430,1917,2076$ \\
\hline TstI & CACNNNNNNTCC & 6 & three_prime & 4 & $471,503,2055,2087$ \\
\hline CfrI & YGGCCR & 6 & five_prime & 5 & $1181,1475,1657,1750,2121$ \\
\hline FokI & GGATG & 5 & five_prime & 5 & $292,405,514,1785,2092$ \\
\hline SmlI & CTYRAG & 6 & five_prime & 5 & $209,468,1701,1816,1949$ \\
\hline TatI & WGTACW & 6 & five_prime & 5 & $410,438,715,1829,1978$ \\
\hline TfiI & GAWTC & 5 & five_prime & 5 & $755,1275,1895,1971,2139$ \\
\hline TspRI & CASTG & 5 & three_prime & 5 & $1064,1517,1718,1855,1871$ \\
\hline AgsI & TTSAA & 5 & three_prime & 6 & $127,396,731,1310,1400,2133$ \\
\hline $\mathrm{BcgI}$ & CGANNNNNNTGC & 6 & three_prime & 6 & $156,190,915,949,1041,1075$ \\
\hline HphI & GGTGA & 5 & three_prime & 6 & $373,780,837,1284,1881,2080$ \\
\hline TauI & GCSGC & 5 & three_prime & 6 & $30,696,876,1132,1359,1521$ \\
\hline BccI & CCATC & 5 & five_prime & 7 & $426,535,547,624,963,1299,1647$ \\
\hline EcoRII & CCWGG & 5 & five_prime & 7 & $707,771,996,1003,1267,1608,1823$ \\
\hline FauI & CCCGC & 5 & five_prime & 7 & $32,265,662,693,1205,1548,2099$ \\
\hline Нpy99I & CGWCG & 5 & three_prime & 7 & $747,811,897,945,1575,1767,1904$ \\
\hline SduI & GDGCHC & 6 & three_prime & 7 & $179,255,353,492,671,1038,1074$ \\
\hline BbvI & GCAGC & 5 & five_prime & 8 & $528,1139,1294,1304,1436,1682,1709,1795$ \\
\hline TseI & GCWGC & 5 & five_prime & 8 & $516,1152,1282,1317,1449,1695,1722,1783$ \\
\hline BseRI & GAGGAG & 6 & three_prime & 8 & $54,624,627,924,960,1131,1610,1613$ \\
\hline SfaNI & GCATC & 5 & five_prime & 9 & $27,748,887,1186,1394,1460,1493,1747,2213$ \\
\hline MboII & GAAGA & 5 & three_prime & 9 & $764,822,939,998,1571,1895,2095,2185,2303$ \\
\hline
\end{tabular}


Samia Abd Allah Abdal-Aziz and Safaa M. Ali / OnLine Journal of Biological Sciences 2021, 21 (1): 69.119 DOI: 10.3844/ojbsci.2021.69.119

Table 16: Restriction digestion of protease gene from XM_032052352.1 Aspergillus pseudotamarii

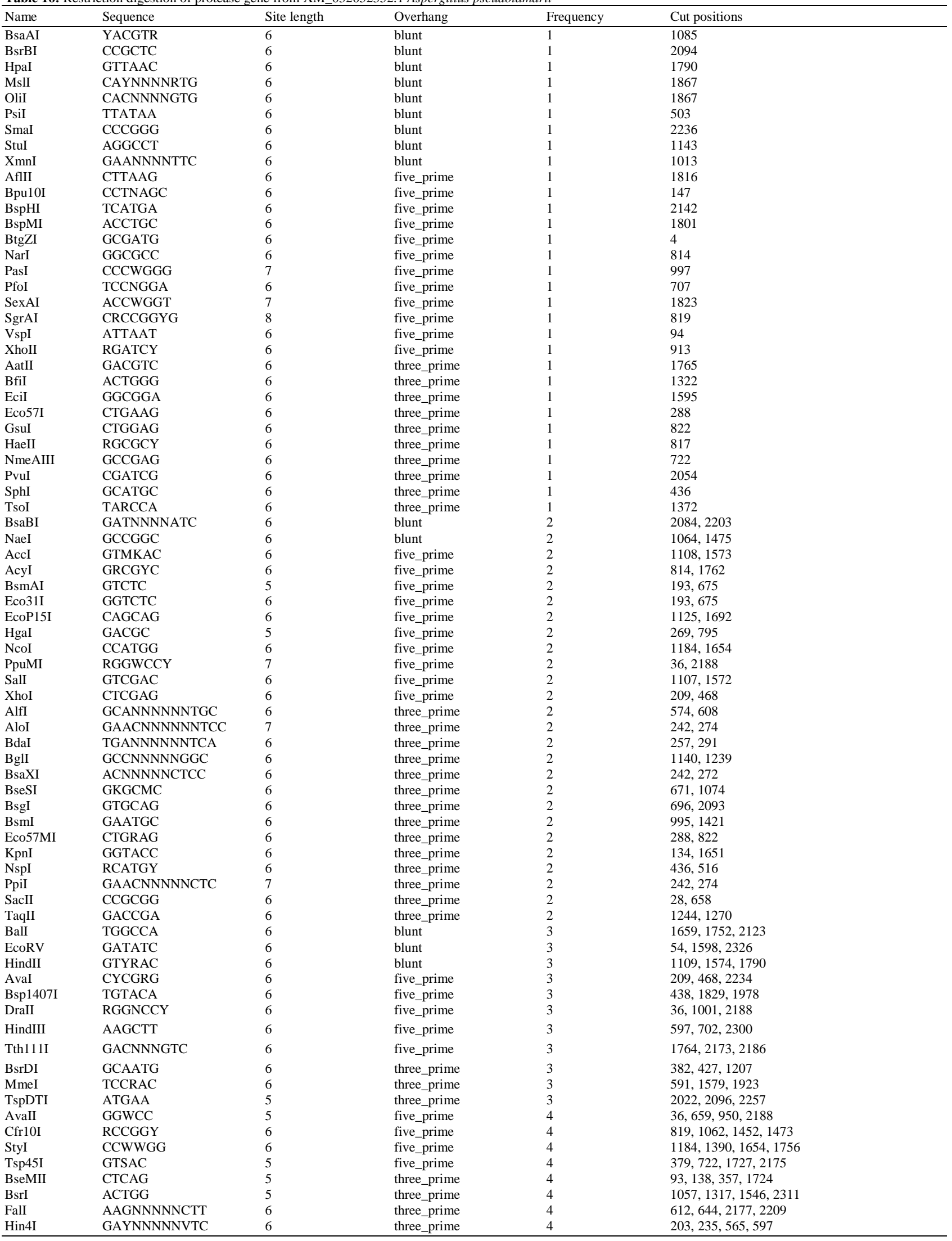


Samia Abd Allah Abdal-Aziz and Safaa M. Ali / OnLine Journal of Biological Sciences 2021, 21 (1): 69.119 DOI: 10.3844/ojbsci.2021.69.119

\begin{tabular}{|c|c|c|c|c|c|}
\hline TspGWI & ACGGA & 5 & three_prime & 4 & $273,1430,1917,2076$ \\
\hline TstI & CACNNNNNNTCC & 6 & three_prime & 4 & $471,503,2055,2087$ \\
\hline CfrI & YGGCCR & 6 & five_prime & 5 & $1181,1475,1657,1750,2121$ \\
\hline SmlI & CTYRAG & 6 & five_prime & 5 & $209,468,1701,1816,1949$ \\
\hline TatI & WGTACW & 6 & five_prime & 5 & $410,438,715,1829,1978$ \\
\hline TfiI & GAWTC & 5 & five_prime & 5 & $755,1275,1895,1971,2139$ \\
\hline TspRI & CASTG & 5 & three_prime & 5 & $1064,1517,1718,1855,1871$ \\
\hline $\mathrm{BcgI}$ & CGANNNNNNTGC & 6 & three_prime & 6 & $156,190,915,949,1041,1075$ \\
\hline $\mathrm{HphI}$ & GGTGA & 5 & three_prime & 6 & $373,780,837,1284,1881,2080$ \\
\hline TauI & GCSGC & 5 & three_prime & 6 & $30,696,876,1132,1359,1521$ \\
\hline BccI & CCATC & 5 & five_prime & 7 & $426,535,547,624,963,1299,1647$ \\
\hline EcoRII & CCWGG & 5 & five_prime & 7 & $707,771,996,1003,1267,1608,1823$ \\
\hline FauI & CCCGC & 5 & five_prime & 7 & $32,265,662,693,1205,1548,2099$ \\
\hline Нpy99I & CGWCG & 5 & three_prime & 7 & $747,811,897,945,1575,1767,1904$ \\
\hline SduI & GDGCHC & 6 & three_prime & 7 & $179,255,353,492,671,1038,1074$ \\
\hline BbvI & GCAGC & 5 & five_prime & 8 & $528,1139,1294,1304,1436,1682,1709,1795$ \\
\hline BseRI & GAGGAG & 6 & three_prime & 8 & $54,624,627,924,960,1131,1610,1613$ \\
\hline SfaNI & GCATC & 5 & five_prime & 9 & $27,748,887,1186,1394,1460,1493,1747,2213$ \\
\hline MboII & GAAGA & 5 & three_prime & 9 & $764,822,939,998,1571,1895,2095,2185,2303$ \\
\hline
\end{tabular}

Table 17: Restriction digestion of protease gene from AY590135.1 Aspergillus viridinutans strain MK246

\begin{tabular}{|c|c|c|c|c|c|}
\hline Name & Sequence & Site length & Overhang & Frequency & Cut positions \\
\hline$\overline{\text { BtrI }}$ & CACGTC & 6 & blunt & 1 & 384 \\
\hline Eco47III & AGCGCT & 6 & blunt & 1 & 330 \\
\hline NaeI & GCCGGC & 6 & blunt & 1 & 96 \\
\hline ScaI & AGTACT & 6 & blunt & 1 & 150 \\
\hline AcyI & GRCGYC & 6 & five_prime & 1 & 260 \\
\hline AvaII & GGWCC & 5 & five_prime & 1 & 219 \\
\hline Bpu10I & CCTNAGC & 6 & five_prime & 1 & 1183 \\
\hline BsmAI & GTCTC & 5 & five_prime & 1 & 1110 \\
\hline BspHI & TCATGA & 6 & five_prime & 1 & 172 \\
\hline BstEII & GGTNACC & 6 & five_prime & 1 & 430 \\
\hline BtgZI & GCGATG & 6 & five_prime & 1 & 53 \\
\hline Cfr10I & RCCGGY & 6 & five_prime & 1 & 94 \\
\hline Eco31I & GGTCTC & 6 & five_prime & 1 & 1110 \\
\hline FauI & CCCGC & 5 & five_prime & 1 & 954 \\
\hline FokI & GGATG & 5 & five_prime & 1 & 390 \\
\hline NarI & GGCGCC & 6 & five_prime & 1 & 260 \\
\hline NdeI & CATATG & 6 & five_prime & 1 & 829 \\
\hline SmlI & CTYRAG & 6 & five_prime & 1 & 1107 \\
\hline XhoI & CTCGAG & 6 & five_prime & 1 & 1107 \\
\hline BciVI & GTATCC & 6 & three_prime & 1 & 816 \\
\hline BfiI & ACTGGG & 6 & three_prime & 1 & 591 \\
\hline BseMII & CTCAG & 5 & three_prime & 1 & 169 \\
\hline BsrDI & GCAATG & 6 & three_prime & 1 & 835 \\
\hline BstXI & CCANNNNNNTGG & 6 & three_prime & 1 & 1080 \\
\hline BtsI & GCAGTG & 6 & three_prime & 1 & 1030 \\
\hline EciI & GGCGGA & 6 & three_prime & 1 & 1001 \\
\hline Eco57MI & CTGRAG & 6 & three_prime & 1 & 31 \\
\hline GsuI & CTGGAG & 6 & three_prime & 1 & 31 \\
\hline HphI & GGTGA & 5 & three_prime & 1 & 1166 \\
\hline KpnI & GGTACC & 6 & three_prime & 1 & 476 \\
\hline SduI & GDGCHC & 6 & three_prime & 1 & 307 \\
\hline BalI & TGGCCA & 6 & blunt & 2 & 502,1065 \\
\hline BsrBI & CCGCTC & 6 & blunt & 2 & 903,1140 \\
\hline $\mathrm{XmnI}$ & GAANNNNTTC & 6 & blunt & 2 & 753,766 \\
\hline AsuII & TTCGAA & 6 & five_prime & 2 & 619,817 \\
\hline AvaI & CYCGRG & 6 & five_prime & 2 & 1107,1118 \\
\hline PleI & GAGTC & 5 & five_prime & 2 & 552,647 \\
\hline Tth111I & GACNNNGTC & 6 & five_prime & 2 & 223,445 \\
\hline AjuI & GAANNNNNNNTTGG & 7 & three_prime & 2 & 751,783 \\
\hline AlfI & GCANNNNNNTGC & 6 & three_prime & 2 & 643,677 \\
\hline BaeI & ACNNNNGTAYC & 7 & three_prime & 2 & 788,821 \\
\hline BplI & GAGNNNNNCTC & 6 & three_prime & 2 & 1101,1133 \\
\hline BseRI & GAGGAG & 6 & three_prime & 2 & 143,229 \\
\hline FalI & AAGNNNNNCTT & 6 & three_prime & 2 & 754,786 \\
\hline HaeIV & GAYNNNNNRTC & 6 & three_prime & 2 & 641,675 \\
\hline MmeI & TCCRAC & 6 & three_prime & 2 & 985,1051 \\
\hline TspDTI & ATGAA & 5 & three_prime & 2 & 161,846 \\
\hline
\end{tabular}


Samia Abd Allah Abdal-Aziz and Safaa M. Ali / OnLine Journal of Biological Sciences 2021, 21 (1): 69.119 DOI: 10.3844/ojbsci.2021.69.119

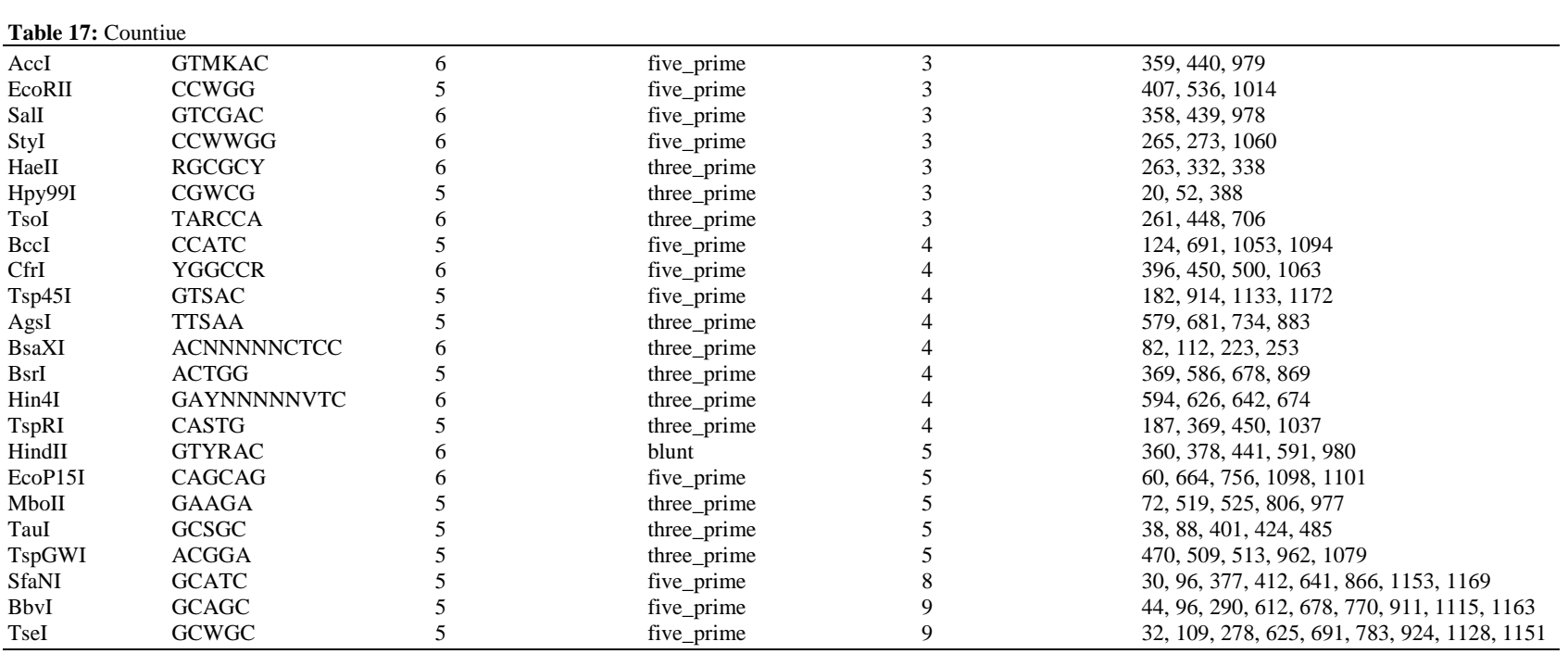

Table 18: Restriction digestion of protease gene from AY590136.1 Aspergillus viridinutans strain MK284

\begin{tabular}{|c|c|c|c|c|c|}
\hline Name & Sequence & Site Length & Overhang & Frequency & Cut Positions \\
\hline$\overline{\text { BtrI }}$ & CACGTC & 6 & blunt & 1 & 384 \\
\hline Eco47III & AGCGCT & 6 & blunt & 1 & 330 \\
\hline NaeI & GCCGGC & 6 & blunt & 1 & 96 \\
\hline ScaI & AGTACT & 6 & blunt & 1 & 150 \\
\hline $\mathrm{XmnI}$ & GAANNNNTTC & 6 & blunt & 1 & 753 \\
\hline AcyI & GRCGYC & 6 & five_prime & 1 & 260 \\
\hline AsuII & TTCGAA & 6 & five_prime & 1 & 817 \\
\hline Bpu10I & CCTNAGC & 6 & five_prime & 1 & 1183 \\
\hline BseYI & CCCAGC & 6 & five_prime & 1 & 893 \\
\hline BsmAI & GTCTC & 5 & five_prime & 1 & 1110 \\
\hline BspHI & TCATGA & 6 & five_prime & 1 & 172 \\
\hline BspMI & ACCTGC & 6 & five_prime & 1 & 1133 \\
\hline BtgZI & GCGATG & 6 & five_prime & 1 & 53 \\
\hline Cfr10I & RCCGGY & 6 & five_prime & 1 & 94 \\
\hline DraII & RGGNCCY & 6 & five_prime & 1 & 1122 \\
\hline Eco31I & GGTCTC & 6 & five_prime & 1 & 1110 \\
\hline FauI & CCCGC & 5 & five_prime & 1 & 954 \\
\hline NarI & GGCGCC & 6 & five_prime & 1 & 260 \\
\hline PpuMI & RGGWCCY & 7 & five_prime & 1 & 1122 \\
\hline TatI & WGTACW & 6 & five_prime & 1 & 148 \\
\hline TfiI & GAWTC & 5 & five_prime & 1 & 1112 \\
\hline XhoI & CTCGAG & 6 & five_prime & 1 & 1107 \\
\hline BciVI & GTATCC & 6 & three_prime & 1 & 816 \\
\hline BfiI & ACTGGG & 6 & three_prime & 1 & 591 \\
\hline BseMII & CTCAG & 5 & three_prime & 1 & 169 \\
\hline BsrDI & GCAATG & 6 & three_prime & 1 & 835 \\
\hline BstXI & CCANNNNNNTGG & 6 & three_prime & 1 & 1080 \\
\hline BtsI & GCAGTG & 6 & three_prime & 1 & 1030 \\
\hline EciI & GGCGGA & 6 & three_prime & 1 & 1001 \\
\hline Eco57MI & CTGRAG & 6 & three_prime & 1 & 31 \\
\hline GsuI & CTGGAG & 6 & three_prime & 1 & 31 \\
\hline HphI & GGTGA & 5 & three_prime & 1 & 1166 \\
\hline KpnI & GGTACC & 6 & three_prime & 1 & 476 \\
\hline SduI & GDGCHC & 6 & three_prime & 1 & 307 \\
\hline BalI & TGGCCA & 6 & blunt & 2 & 502,1065 \\
\hline BsrBI & CCGCTC & 6 & blunt & 2 & 903,1140 \\
\hline AvaI & CYCGRG & 6 & five_prime & 2 & 1107,1118 \\
\hline StyI & CCWWGG & 6 & five_prime & 2 & 265,1060 \\
\hline Tth111I & GACNNNGTC & 6 & five_prime & 2 & 223,445 \\
\hline AlfI & GCANNNNNNTGC & 6 & three_prime & 2 & 643,677 \\
\hline BaeI & ACNNNNGTAYC & 7 & three_prime & 2 & 788,821 \\
\hline $\mathrm{BcgI}$ & CGANNNNNNTGC & 6 & three_prime & 2 & 420,454 \\
\hline BplI & GAGNNNNNCTC & 6 & three_prime & 2 & 1101,1133 \\
\hline BseRI & GAGGAG & 6 & three_prime & 2 & 143,229 \\
\hline FalI & AAGNNNNNCTT & 6 & three_prime & 2 & 754,786 \\
\hline HaeIV & GAYNNNNNRTC & 6 & three_prime & 2 & 641,675 \\
\hline MmeI & TCCRAC & 6 & three_prime & 2 & 985,1051 \\
\hline
\end{tabular}


Samia Abd Allah Abdal-Aziz and Safaa M. Ali / OnLine Journal of Biological Sciences 2021, 21 (1): 69.119 DOI: 10.3844/ojbsci.2021.69.119

\begin{tabular}{|c|c|c|c|c|c|}
\hline TsoI & TARCCA & 6 & three_prime & 2 & 261,706 \\
\hline TspDTI & ATGAA & 5 & three_prime & 2 & 161,846 \\
\hline AccI & GTMKAC & 6 & five_prime & 3 & $359,440,979$ \\
\hline HgaI & GACGC & 5 & five_prime & 3 & $4,169,435$ \\
\hline SalI & GTCGAC & 6 & five_prime & 3 & $358,439,978$ \\
\hline AgsI & TTSAA & 5 & three_prime & 3 & $579,681,734$ \\
\hline BsrI & ACTGG & 5 & three_prime & 3 & $369,586,678$ \\
\hline TspRI & CASTG & 5 & three_prime & 3 & $187,369,1037$ \\
\hline HindII & GTYRAC & 6 & blunt & 4 & $360,378,441,980$ \\
\hline $\mathrm{BccI}$ & CCATC & 5 & five_prime & 4 & $124,691,1053,1094$ \\
\hline CfrI & YGGCCR & 6 & five_prime & 4 & $396,450,500,1063$ \\
\hline Tsp45I & GTSAC & 5 & five_prime & 4 & $182,914,1133,1172$ \\
\hline BsaXI & ACNNNNNCTCC & 6 & three_prime & 4 & $82,112,223,253$ \\
\hline Hin4I & GAYNNNNNVTC & 6 & three_prime & 4 & $594,626,642,674$ \\
\hline Hрy99I & CGWCG & 5 & three_prime & 4 & $20,52,388,451$ \\
\hline TspGWI & ACGGA & 5 & three_prime & 4 & $470,509,513,1079$ \\
\hline MboII & GAAGA & 5 & three_prime & 5 & $72,519,525,806,977$ \\
\hline TauI & GCSGC & 5 & three_prime & 6 & $38,88,401,424,431,485$ \\
\hline SfaNI & GCATC & 5 & five_prime & 8 & $30,96,377,412,641,866,1153,1169$ \\
\hline BbvI & GCAGC & 5 & five_prime & 10 & $44,96,290,573,612,678,770,911,1115,1163$ \\
\hline TseI & GCWGC & 5 & five_prime & 10 & $32,109,278,586,625,691,783,924,1128,1151$ \\
\hline
\end{tabular}

Table 19: Restriction digestion of protease gene from MG867728.1 Aspergillus sojae

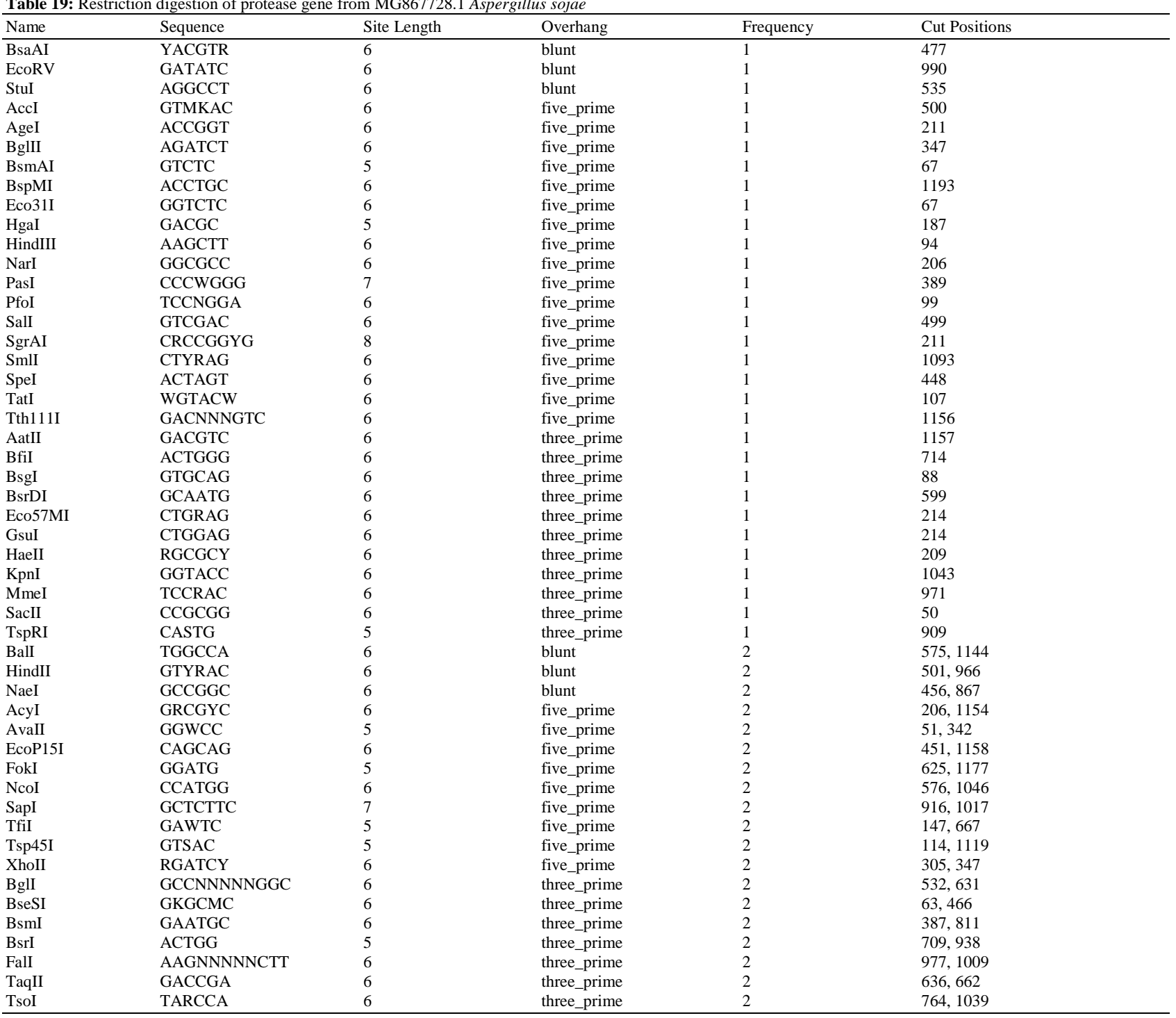


Samia Abd Allah Abdal-Aziz and Safaa M. Ali / OnLine Journal of Biological Sciences 2021, 21 (1): 69.119 DOI: 10.3844/ojbsci.2021.69.119

\begin{tabular}{|c|c|c|c|c|c|}
\hline $\mathrm{XmnI}$ & GAANNNNTTC & 6 & blunt & 3 & $95,405,811$ \\
\hline CfrI & YGGCCR & 6 & five_prime & 3 & $573,867,1142$ \\
\hline FauI & CCCGC & 5 & five_prime & 3 & $54,85,1119$ \\
\hline AgsI & TTSAA & 5 & three_prime & 3 & $123,702,792$ \\
\hline HphI & GGTGA & 5 & three_prime & 3 & $172,229,676$ \\
\hline SduI & GDGCHC & 6 & three_prime & 3 & $63,430,466$ \\
\hline $\mathrm{BcgI}$ & CGANNNNNNTGC & 6 & three_prime & 4 & $307,341,433,467$ \\
\hline Cfr10I & RCCGGY & 6 & five_prime & 5 & $211,454,547,844,865$ \\
\hline EcoRII & CCWGG & 5 & five_prime & 5 & $99,163,388,659,1000$ \\
\hline BseRI & GAGGAG & 6 & three_prime & 5 & $16,19,316,352,523$ \\
\hline TauI & GCSGC & 5 & three_prime & 5 & $88,268,524,913,935$ \\
\hline BccI & CCATC & 5 & five_prime & 6 & $16,304,355,691,1039,1099$ \\
\hline SfaNI & GCATC & 5 & five_prime & 6 & $279,647,786,852,885,1139$ \\
\hline MboII & GAAGA & 5 & three_prime & 6 & $214,331,390,933,963,1004$ \\
\hline BbvI & GCAGC & 5 & five_prime & 7 & $531,686,696,735,828,1074,1101$ \\
\hline TseI & GCWGC & 5 & five_prime & 7 & $544,674,709,748,841,1087,1114$ \\
\hline
\end{tabular}

Table 20: Restriction digestion of protease gene from XM_032073130.1 Aspergillus caelatus

\begin{tabular}{|c|c|c|c|c|c|}
\hline Name & Sequence & Site length & Overhang & Frequency & Cut positions \\
\hline BsaAI & YACGTR & 6 & blunt & 1 & 477 \\
\hline EcoRV & GATATC & 6 & blunt & 1 & 990 \\
\hline $\mathrm{HpaI}$ & GTTAAC & 6 & blunt & 1 & 1182 \\
\hline NaeI & GCCGGC & 6 & blunt & 1 & 456 \\
\hline StuI & AGGCCT & 6 & blunt & 1 & 535 \\
\hline $\mathrm{XmnI}$ & GAANNNNTTC & 6 & blunt & 1 & 405 \\
\hline AccI & GTMKAC & 6 & five_prime & 1 & 500 \\
\hline BsmAI & GTCTC & 5 & five_prime & 1 & 67 \\
\hline BspMI & ACCTGC & 6 & five_prime & 1 & 1193 \\
\hline DraII & RGGNCCY & 6 & five_prime & 1 & 393 \\
\hline Eco31I & GGTCTC & 6 & five_prime & 1 & 67 \\
\hline EcoP15I & CAGCAG & 6 & five_prime & 1 & 1084 \\
\hline HgaI & GACGC & 5 & five_prime & 1 & 187 \\
\hline HindIII & AAGCTT & 6 & five_prime & 1 & 94 \\
\hline NarI & GGCGCC & 6 & five_prime & 1 & 206 \\
\hline NcoI & CCATGG & 6 & five_prime & 1 & 1046 \\
\hline PasI & CCCWGGG & 7 & five_prime & 1 & 389 \\
\hline PfoI & TCCNGGA & 6 & five_prime & 1 & 99 \\
\hline TatI & WGTACW & 6 & five_prime & 1 & 107 \\
\hline Tth111I & GACNNNGTC & 6 & five_prime & 1 & 1156 \\
\hline XhoII & RGATCY & 6 & five_prime & 1 & 305 \\
\hline AatII & GACGTC & 6 & three_prime & 1 & 1157 \\
\hline BfiI & ACTGGG & 6 & three_prime & 1 & 714 \\
\hline BsgI & GTGCAG & 6 & three_prime & 1 & 88 \\
\hline BsmI & GAATGC & 6 & three_prime & 1 & 387 \\
\hline BsrDI & GCAATG & 6 & three_prime & 1 & 599 \\
\hline EciI & GGCGGA & 6 & three_prime & 1 & 987 \\
\hline Eco57MI & CTGRAG & 6 & three_prime & 1 & 214 \\
\hline GsuI & CTGGAG & 6 & three_prime & 1 & 214 \\
\hline HaeII & RGCGCY & 6 & three_prime & 1 & 209 \\
\hline KpnI & GGTACC & 6 & three_prime & 1 & 1043 \\
\hline MmeI & TCCRAC & 6 & three_prime & 1 & 971 \\
\hline NmeAIII & GCCGAG & 6 & three_prime & 1 & 114 \\
\hline SacII & CCGCGG & 6 & three_prime & 1 & 50 \\
\hline TsoI & TARCCA & 6 & three_prime & 1 & 764 \\
\hline AcyI & GRCGYC & 6 & five_prime & 2 & 206,1154 \\
\hline Tsp45I & GTSAC & 5 & five_prime & 2 & 114,1119 \\
\hline BglI & GCCNNNNNGGC & 6 & three_prime & 2 & 532,631 \\
\hline BseSI & GKGCMC & 6 & three_prime & 2 & 63,466 \\
\hline TspRI & CASTG & 5 & three_prime & 2 & 456,909 \\
\hline BalI & TGGCCA & 6 & blunt & 3 & $869,1051,1144$ \\
\hline HindII & GTYRAC & 6 & blunt & 3 & $501,966,1182$ \\
\hline Cfr10I & RCCGGY & 6 & five_prime & 3 & $454,547,844$ \\
\hline CfrI & YGGCCR & 6 & five_prime & 3 & $867,1049,1142$ \\
\hline FauI & CCCGC & 5 & five_prime & 3 & $54,85,597$ \\
\hline StyI & CCWWGG & 6 & five_prime & 3 & $782,1046,1148$ \\
\hline AgsI & TTSAA & 5 & three_prime & 3 & $123,702,792$ \\
\hline BsrI & ACTGG & 5 & three_prime & 3 & $449,709,938$ \\
\hline HphI & GGTGA & 5 & three_prime & 3 & $172,229,676$ \\
\hline SduI & GDGCHC & 6 & three_prime & 3 & $63,430,466$ \\
\hline TaqII & GACCGA & 6 & three_prime & 4 & $562,588,636,662$ \\
\hline
\end{tabular}


Samia Abd Allah Abdal-Aziz and Safaa M. Ali / OnLine Journal of Biological Sciences 2021, 21 (1): 69.119 DOI: 10.3844/ojbsci.2021.69.119

\begin{tabular}{|c|c|c|c|c|c|}
\hline Hpy99I & CGWCG & 5 & three_prime & 5 & $139,203,289,337,1159$ \\
\hline MboII & GAAGA & 5 & three_prime & 5 & $156,214,331,390,963$ \\
\hline TauI & GCSGC & 5 & three_prime & 5 & $88,215,268,524,913$ \\
\hline EcoRII & CCWGG & 5 & five_prime & 6 & $99,163,388,395,659,1000$ \\
\hline $\mathrm{BcgI}$ & CGANNNNNNTGC & 6 & three_prime & 6 & $307,341,433,467,1093,1127$ \\
\hline SfaNI & GCATC & 5 & five_prime & 7 & $140,279,578,786,852,885,1139$ \\
\hline BseRI & GAGGAG & 6 & three_prime & 7 & $16,19,316,352,523,1002,1005$ \\
\hline TseI & GCWGC & 5 & five_prime & 8 & $544,674,709,748,841,1087,1114,1175$ \\
\hline
\end{tabular}

Table 21: Restriction digestion of protease gene from XM_001266851.1 Aspergillus fischeri

\begin{tabular}{|c|c|c|c|c|c|}
\hline Name & Sequence & Site length & Overhang & Frequency & Cut positions \\
\hline$\overline{\text { BsrBI }}$ & CCGCTC & 6 & blunt & 1 & 889 \\
\hline BtrI & CACGTC & 6 & blunt & 1 & 507 \\
\hline Eco47III & AGCGCT & 6 & blunt & 1 & 453 \\
\hline EcoRV & GATATC & 6 & blunt & 1 & 990 \\
\hline NaeI & GCCGGC & 6 & blunt & 1 & 276 \\
\hline PvuII & CAGCTG & 6 & blunt & 1 & 1140 \\
\hline $\mathrm{XmnI}$ & GAANNNNTTC & 6 & blunt & 1 & 811 \\
\hline AscI & GGCGCGCC & 8 & five_prime & 1 & 59 \\
\hline AvaI & CYCGRG & 6 & five_prime & 1 & 1093 \\
\hline AvaII & GGWCC & 5 & five_prime & 1 & 342 \\
\hline Bpu10I & CCTNAGC & 6 & five_prime & 1 & 1169 \\
\hline BsePI & GCGCGC & 6 & five_prime & 1 & 59 \\
\hline BtgZI & GCGATG & 6 & five_prime & 1 & 512 \\
\hline Cfr10I & RCCGGY & 6 & five_prime & 1 & 274 \\
\hline Eco31I & GGTCTC & 6 & five_prime & 1 & 1096 \\
\hline FauI & CCCGC & 5 & five_prime & 1 & 940 \\
\hline HindIII & AAGCTT & 6 & five_prime & 1 & 1186 \\
\hline NarI & GGCGCC & 6 & five_prime & 1 & 383 \\
\hline TatI & WGTACW & 6 & five_prime & 1 & 107 \\
\hline TfiI & GAWTC & 5 & five_prime & 1 & 96 \\
\hline Tth111I & GACNNNGTC & 6 & five_prime & 1 & 568 \\
\hline XhoI & CTCGAG & 6 & five_prime & 1 & 1093 \\
\hline BfiI & ACTGGG & 6 & three_prime & 1 & 714 \\
\hline BglI & GCCNNNNNGGC & 6 & three_prime & 1 & 1106 \\
\hline BseRI & GAGGAG & 6 & three_prime & 1 & 352 \\
\hline BseSI & GKGCMC & 6 & three_prime & 1 & 133 \\
\hline BsrDI & GCAATG & 6 & three_prime & 1 & 1209 \\
\hline BtsI & GCAGTG & 6 & three_prime & 1 & 1016 \\
\hline DrdI & GACNNNNNNGTC & 6 & three_prime & 1 & 823 \\
\hline EciI & GGCGGA & 6 & three_prime & 1 & 987 \\
\hline Eco57MI & CTGRAG & 6 & three_prime & 1 & 214 \\
\hline GsuI & CTGGAG & 6 & three_prime & 1 & 214 \\
\hline HphI & GGTGA & 5 & three_prime & 1 & 1152 \\
\hline KpnI & GGTACC & 6 & three_prime & 1 & 599 \\
\hline SduI & GDGCHC & 6 & three_prime & 1 & 133 \\
\hline TsoI & TARCCA & 6 & three_prime & 1 & 764 \\
\hline EcoP15I & CAGCAG & 6 & five_prime & 2 & 814,1084 \\
\hline $\mathrm{NcoI}$ & CCATGG & 6 & five_prime & 2 & 388,1046 \\
\hline SalI & GTCGAC & 6 & five_prime & 2 & 481,964 \\
\hline AlfI & GCANNNNNNTGC & 6 & three_prime & 2 & 6,40 \\
\hline $\mathrm{BcgI}$ & CGANNNNNNTGC & 6 & three_prime & 2 & 93,127 \\
\hline BplI & GAGNNNNNCTC & 6 & three_prime & 2 & 1087,1119 \\
\hline BseMII & CTCAG & 5 & three_prime & 2 & 103,1183 \\
\hline BsrI & ACTGG & 5 & three_prime & 2 & 492,709 \\
\hline FalI & AAGNNNNNCTT & 6 & three_prime & 2 & 812,844 \\
\hline Hin4I & GAYNNNNNVTC & 6 & three_prime & 2 & 717,749 \\
\hline MmeI & TCCRAC & 6 & three_prime & 2 & 971,1037 \\
\hline BalI & TGGCCA & 6 & blunt & 3 & $625,1051,1144$ \\
\hline HgaI & GACGC & 5 & five_prime & 3 & $187,370,558$ \\
\hline PleI & GAGTC & 5 & five_prime & 3 & $159,675,770$ \\
\hline StyI & CCWWGG & 6 & five_prime & 3 & $30,388,1046$ \\
\hline Tsp45I & GTSAC & 5 & five_prime & 3 & $114,1119,1158$ \\
\hline AgsI & TTSAA & 5 & three_prime & 3 & $123,702,792$ \\
\hline HaelI & RGCGCY & 6 & three_prime & 3 & $386,455,461$ \\
\hline HindII & GTYRAC & 6 & blunt & 4 & $483,501,564,966$ \\
\hline CfrI & YGGCCR & 6 & five_prime & 4 & $573,623,1049,1142$ \\
\hline BsaXI & ACNNNNNCTCC & 6 & three_prime & 4 & $262,292,940,970$ \\
\hline TspRI & CASTG & 5 & three_prime & 4 & $177,492,864,1023$ \\
\hline BccI & CCATC & 5 & five_prime & 5 & $235,304,691,1039,1080$ \\
\hline
\end{tabular}


Samia Abd Allah Abdal-Aziz and Safaa M. Ali / OnLine Journal of Biological Sciences 2021, 21 (1): 69.119 DOI: 10.3844/ojbsci.2021.69.119

\begin{tabular}{|c|c|c|c|c|c|}
\hline EcoRII & CCWGG & 5 & five_prime & 5 & $68,99,191,659,1000$ \\
\hline Нpy99I & CGWCG & 5 & three_prime & 5 & $203,511,574,820,841$ \\
\hline MboII & GAAGA & 5 & three_prime & 5 & $105,252,642,648,963$ \\
\hline TauI & GCSGC & 5 & three_prime & 5 & $218,268,524,547,608$ \\
\hline TspGWI & ACGGA & 5 & three_prime & 5 & $593,632,636,948,1065$ \\
\hline SfaNI & GCATC & 5 & five_prime & 6 & $126,195,500,535,852,1139$ \\
\hline BbvI & GCAGC & 5 & five_prime & 12 & $11,72,151,413,566,696,735,828,897,1101,1149,1187$ \\
\hline TseI & GCWGC & 5 & five_prime & 12 & $24,85,139,401,554,709,748,841,910,1114,1137,1175$ \\
\hline
\end{tabular}

Table 22: Restriction digestion of protease gene from >XM_024833434.1 Aspergillus campestris

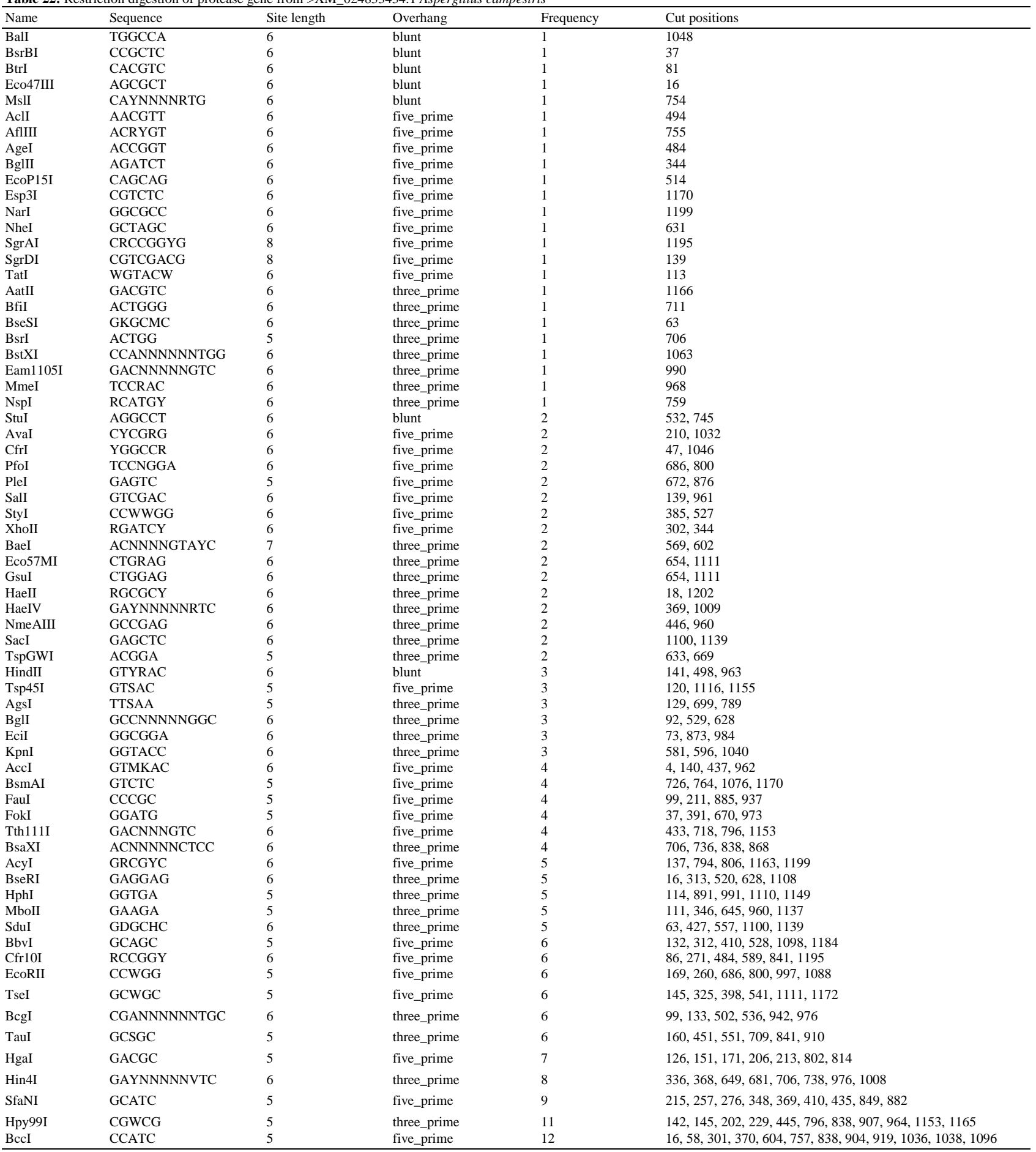


Samia Abd Allah Abdal-Aziz and Safaa M. Ali / OnLine Journal of Biological Sciences 2021, 21 (1): 69.119 DOI: 10.3844/ojbsci.2021.69.119

Table 23: Restriction digestion of protease gene from XM_024825725.1 Aspergillus novofumigatus

\begin{tabular}{|c|c|c|c|c|c|}
\hline Name & Sequence & Site Length & Overhang & Frequency & Cut Positions \\
\hline BsrBI & CCGCTC & 6 & blunt & 1 & 889 \\
\hline BtrI & CACGTC & 6 & blunt & 1 & 507 \\
\hline EcoRV & GATATC & 6 & blunt & 1 & 990 \\
\hline PvuII & CAGCTG & 6 & blunt & 1 & 1140 \\
\hline $\mathrm{XmnI}$ & GAANNNNTTC & 6 & blunt & 1 & 811 \\
\hline AgeI & ACCGGT & 6 & five_prime & 1 & 1162 \\
\hline AscI & GGCGCGCC & 8 & five_prime & 1 & 59 \\
\hline AsuII & TTCGAA & 6 & five_prime & 1 & 868 \\
\hline AvaI & CYCGRG & 6 & five_prime & 1 & 1093 \\
\hline AvaII & GGWCC & 5 & five_prime & 1 & 342 \\
\hline BsePI & GCGCGC & 6 & five_prime & 1 & 59 \\
\hline BtgZI & GCGATG & 6 & five_prime & 1 & 512 \\
\hline Eco31I & GGTCTC & 6 & five_prime & 1 & 1096 \\
\hline FauI & CCCGC & 5 & five_prime & 1 & 940 \\
\hline HindIII & AAGCTT & 6 & five_prime & 1 & 1186 \\
\hline NarI & GGCGCC & 6 & five_prime & 1 & 383 \\
\hline PfoI & TCCNGGA & 6 & five_prime & 1 & 68 \\
\hline SgrAI & CRCCGGYG & 8 & five_prime & 1 & 454 \\
\hline TfiI & GAWTC & 5 & five_prime & 1 & 96 \\
\hline Tth111I & GACNNNGTC & 6 & five_prime & 1 & 568 \\
\hline XhoI & CTCGAG & 6 & five_prime & 1 & 1093 \\
\hline BfiI & ACTGGG & 6 & three_prime & 1 & 714 \\
\hline BseRI & GAGGAG & 6 & three_prime & 1 & 352 \\
\hline BseSI & GKGCMC & 6 & three_prime & 1 & 133 \\
\hline BsmI & GAATGC & 6 & three_prime & 1 & 3 \\
\hline BsrDI & GCAATG & 6 & three_prime & 1 & 1209 \\
\hline BtsI & GCAGTG & 6 & three_prime & 1 & 1016 \\
\hline Eco57MI & CTGRAG & 6 & three_prime & 1 & 214 \\
\hline GsuI & CTGGAG & 6 & three_prime & 1 & 214 \\
\hline KpnI & GGTACC & 6 & three_prime & 1 & 599 \\
\hline MmeI & TCCRAC & 6 & three_prime & 1 & 971 \\
\hline TsoI & TARCCA & 6 & three_prime & 1 & 764 \\
\hline NaeI & GCCGGC & 6 & blunt & 2 & 276,456 \\
\hline AccI & GTMKAC & 6 & five_prime & 2 & 482,965 \\
\hline AcyI & GRCGYC & 6 & five_prime & 2 & 362,383 \\
\hline BsmAI & GTCTC & 5 & five_prime & 2 & 158,1096 \\
\hline AlfI & GCANNNNNNTGC & 6 & three_prime & 2 & 6,40 \\
\hline BaeI & ACNNNNGTAYC & 7 & three_prime & 2 & 212,245 \\
\hline $\mathrm{BcgI}$ & CGANNNNNNTGC & 6 & three_prime & 2 & 93,127 \\
\hline BplI & GAGNNNNNCTC & 6 & three_prime & 2 & 1087,1119 \\
\hline BseMII & CTCAG & 5 & three_prime & 2 & 103,1183 \\
\hline BstXI & CCANNNNNNTGG & 6 & three_prime & 2 & 592,1066 \\
\hline Hin4I & GAYNNNNNVTC & 6 & three_prime & 2 & 717,749 \\
\hline HphI & GGTGA & 5 & three_prime & 2 & 229,1152 \\
\hline SduI & GDGCHC & 6 & three_prime & 2 & 90,133 \\
\hline BalI & TGGCCA & 6 & blunt & 3 & $625,1051,1144$ \\
\hline Cfr10I & RCCGGY & 6 & five_prime & 3 & $274,454,1162$ \\
\hline PleI & GAGTC & 5 & five_prime & 3 & $159,675,770$ \\
\hline StyI & CCWWGG & 6 & five_prime & 3 & $30,388,1046$ \\
\hline Tsp45I & GTSAC & 5 & five_prime & 3 & $114,1119,1158$ \\
\hline AgsI & TTSAA & 5 & three_prime & 3 & $123,702,792$ \\
\hline BsrI & ACTGG & 5 & three_prime & 3 & $492,709,1148$ \\
\hline HaeII & RGCGCY & 6 & three_prime & 3 & $386,455,461$ \\
\hline HindII & GTYRAC & 6 & blunt & 4 & $483,501,564,966$ \\
\hline BccI & CCATC & 5 & five_prime & 4 & $220,304,691,1080$ \\
\hline CfrI & YGGCCR & 6 & five_prime & 4 & $573,623,1049,1142$ \\
\hline HgaI & GACGC & 5 & five_prime & 4 & $145,187,370,558$ \\
\hline BsaXI & ACNNNNNCTCC & 6 & three_prime & 4 & $262,292,940,970$ \\
\hline FalI & AAGNNNNNCTT & 6 & three_prime & 4 & $812,844,867,899$ \\
\hline EcoRII & CCWGG & 5 & five_prime & 5 & $68,99,191,659,1000$ \\
\hline MboII & GAAGA & 5 & three_prime & 5 & $105,252,642,648,963$ \\
\hline TauI & GCSGC & 5 & three_prime & 5 & $268,524,547,608,751$ \\
\hline TspGWI & ACGGA & 5 & three_prime & 5 & $593,632,636,948,1065$ \\
\hline SfaNI & GCATC & 5 & five_prime & 6 & $195,279,500,535,852,1139$ \\
\hline Нpy99I & CGWCG & 5 & three_prime & 6 & $83,139,203,511,574,841$ \\
\hline TspRI & CASTG & 5 & three_prime & 6 & $177,492,822,864,1023,1155$ \\
\hline BbvI & GCAGC & 5 & five_prime & 10 & $11,151,413,566,696,828,897,1101,1149,1187$ \\
\hline TseI & GCWGC & 5 & five_prime & 10 & $24,139,401,554,709,841,910,1114,1137,1175$ \\
\hline
\end{tabular}


Samia Abd Allah Abdal-Aziz and Safaa M. Ali / OnLine Journal of Biological Sciences 2021, 21 (1): 69.119 DOI: 10.3844/ojbsci.2021.69.119

Table 24: Restriction digestion of protease gene from XM_024813742.1 Aspergillus candidus

\begin{tabular}{|c|c|c|c|c|c|}
\hline Name & Sequence & Site length & Overhang & Frequency & Cut positions \\
\hline BalI & TGGCCA & 6 & blunt & 1 & 1237 \\
\hline BsrBI & CCGCTC & 6 & blunt & 1 & 226 \\
\hline BtrI & CACGTC & 6 & blunt & 1 & 270 \\
\hline Eco47III & AGCGCT & 6 & blunt & 1 & 205 \\
\hline EcoRV & GATATC & 6 & blunt & 1 & 1155 \\
\hline MslI & CAYNNNNRTG & 6 & blunt & 1 & 943 \\
\hline AflII & CTTAAG & 6 & five_prime & 1 & 13 \\
\hline AflIII & ACRYGT & 6 & five_prime & 1 & 944 \\
\hline AgeI & ACCGGT & 6 & five_prime & 1 & 673 \\
\hline ApoI & RAATTY & 6 & five_prime & 1 & 1126 \\
\hline BglII & AGATCT & 6 & five_prime & 1 & 533 \\
\hline EcoP15I & CAGCAG & 6 & five_prime & 1 & 610 \\
\hline EcoRI & GAATTC & 6 & five_prime & 1 & 1126 \\
\hline Esp3I & CGTCTC & 6 & five_prime & 1 & 1359 \\
\hline NarI & GGCGCC & 6 & five_prime & 1 & 1388 \\
\hline PasI & CCCWGGG & 7 & five_prime & 1 & 575 \\
\hline SalI & GTCGAC & 6 & five_prime & 1 & 328 \\
\hline SgrAI & CRCCGGYG & 8 & five_prime & 1 & 1384 \\
\hline SgrDI & CGTCGACG & 8 & five_prime & 1 & 328 \\
\hline SmlI & CTYRAG & 6 & five_prime & 1 & 13 \\
\hline StyI & CCWWGG & 6 & five_prime & 1 & 716 \\
\hline TatI & WGTACW & 6 & five_prime & 1 & 302 \\
\hline TfiI & GAWTC & 5 & five_prime & 1 & 1545 \\
\hline AatII & GACGTC & 6 & three_prime & 1 & 1355 \\
\hline BfiI & ACTGGG & 6 & three_prime & 1 & 900 \\
\hline BseSI & GKGCMC & 6 & three_prime & 1 & 252 \\
\hline BsgI & GTGCAG & 6 & three_prime & 1 & 1516 \\
\hline BsrI & ACTGG & 5 & three_prime & 1 & 895 \\
\hline BstXI & CCANNNNNNTGG & 6 & three_prime & 1 & 1252 \\
\hline NspI & RCATGY & 6 & three_prime & 1 & 948 \\
\hline TspRI & CASTG & 5 & three_prime & 1 & 1540 \\
\hline AclI & AACGTT & 6 & five_prime & 2 & 159,683 \\
\hline AvaI & CYCGRG & 6 & five_prime & 2 & 399,1221 \\
\hline AvaII & GGWCC & 5 & five_prime & 2 & 42,84 \\
\hline CfrI & YGGCCR & 6 & five_prime & 2 & 236,1235 \\
\hline PfoI & TCCNGGA & 6 & five_prime & 2 & 875,989 \\
\hline PleI & GAGTC & 5 & five_prime & 2 & 861,1051 \\
\hline BaeI & ACNNNNGTAYC & 7 & three_prime & 2 & 758,791 \\
\hline BsaXI & ACNNNNNCTCC & 6 & three_prime & 2 & 895,925 \\
\hline EciI & GGCGGA & 6 & three_prime & 2 & 262,1062 \\
\hline HaeII & RGCGCY & 6 & three_prime & 2 & 207,1391 \\
\hline SduI & GDGCHC & 6 & three_prime & 2 & 252,746 \\
\hline TspDTI & ATGAA & 5 & three_prime & 2 & 1400,1494 \\
\hline Tth111I & GACNNNGTC & 6 & five_prime & 3 & $622,907,1342$ \\
\hline $\mathrm{BglI}$ & GCCNNNNNGGC & 6 & three_prime & 3 & $281,718,817$ \\
\hline GsuI & CTGGAG & 6 & three_prime & 3 & $843,1044,1300$ \\
\hline HaeIV & GAYNNNNNRTC & 6 & three_prime & 3 & $558,1198,1341$ \\
\hline KpnI & GGTACC & 6 & three_prime & 3 & $770,785,1229$ \\
\hline MmeI & TCCRAC & 6 & three_prime & 3 & $201,1157,1418$ \\
\hline TspGWI & ACGGA & 5 & three_prime & 3 & $822,858,1479$ \\
\hline HindII & GTYRAC & 6 & blunt & 4 & $157,330,687,1462$ \\
\hline StuI & AGGCCT & 6 & blunt & 4 & $71,721,934,973$ \\
\hline AccI & GTMKAC & 6 & five_prime & 4 & $193,329,626,1520$ \\
\hline AcyI & GRCGYC & 6 & five_prime & 4 & $326,995,1352,1388$ \\
\hline BsmAI & GTCTC & 5 & five_prime & 4 & $915,953,1265,1359$ \\
\hline FauI & CCCGC & 5 & five_prime & 4 & $288,400,1074,1126$ \\
\hline Tsp45I & GTSAC & 5 & five_prime & 4 & $3,309,1305,1344$ \\
\hline XhoII & RGATCY & 6 & five_prime & 4 & $491,533,1284,1323$ \\
\hline
\end{tabular}


Samia Abd Allah Abdal-Aziz and Safaa M. Ali / OnLine Journal of Biological Sciences 2021, 21 (1): 69.119 DOI: 10.3844/ojbsci.2021.69.119

\begin{tabular}{|c|c|c|c|c|c|}
\hline BseRI & GAGGAG & 6 & three_prime & 4 & $64,502,709,817$ \\
\hline Eco57MI & CTGRAG & 6 & three_prime & 4 & $117,843,1044,1300$ \\
\hline HphI & GGTGA & 5 & three_prime & 5 & $303,1080,1180,1299,1338$ \\
\hline BbvI & GCAGC & 5 & five_prime & 6 & $321,501,599,624,1287,1373$ \\
\hline Cfr10I & RCCGGY & 6 & five_prime & 6 & $81,275,673,778,1030,1384$ \\
\hline HgaI & GACGC & 5 & five_prime & 6 & $315,340,360,395,402,1003$ \\
\hline TseI & GCWGC & 5 & five_prime & 6 & $334,514,587,637,1300,1361$ \\
\hline AgsI & TTSAA & 5 & three_prime & 6 & $27,318,450,888,978,1542$ \\
\hline MboII & GAAGA & 5 & three_prime & 6 & $96,123,300,535,1149,1326$ \\
\hline TauI & GCSGC & 5 & three_prime & 6 & $349,733,740,898,1030,1099$ \\
\hline EcoRII & CCWGG & 5 & five_prime & 7 & $358,574,875,968,989,1186,1277$ \\
\hline SfaNI & GCATC & 5 & five_prime & 10 & $404,446,465,537,558,599,624,972,1038,1071$ \\
\hline FokI & GGATG & 5 & five_prime & 11 & $32,95,101,217,226,580,859,1162,1486,1490,1507$ \\
\hline $\mathrm{BcgI}$ & CGANNNNNNTGC & 6 & three_prime & 12 & $274,288,308,322,493,527,619,653,691,725,1131,1165$ \\
\hline Hin4I & GAYNNNNNVTC & 6 & three_prime & 12 & $215,247,525,557,838,870,895,927,1165,1197,1308,1340$ \\
\hline BccI & CCATC & 5 & five_prime & 14 & $116,205,247,490,559,793,946,1027,1093,1108,1225,1227,1468,1489$ \\
\hline
\end{tabular}

Table 25: Restriction digestion of protease gene from XM_025678012.1 Aspergillus costaricaensis

\begin{tabular}{|c|c|c|c|c|c|}
\hline Name & Sequence & Site length & Overhang & Frequency & Cut positions \\
\hline$\overline{\text { BalI }}$ & TGGCCA & 6 & blunt & 1 & 1051 \\
\hline BsaAI & YACGTR & 6 & blunt & 1 & 333 \\
\hline Eco47III & AGCGCT & 6 & blunt & 1 & 453 \\
\hline HindII & GTYRAC & 6 & blunt & 1 & 966 \\
\hline OliI & CACNNNNGTG & 6 & blunt & 1 & 416 \\
\hline PshAI & GACNNNNGTC & 6 & blunt & 1 & 682 \\
\hline SmaI & CCCGGG & 6 & blunt & 1 & 101 \\
\hline AsulI & TTCGAA & 6 & five_prime & 1 & 625 \\
\hline BseYI & CCCAGC & 6 & five_prime & 1 & 1110 \\
\hline BsmAI & GTCTC & 5 & five_prime & 1 & 1131 \\
\hline BspMI & ACCTGC & 6 & five_prime & 1 & 60 \\
\hline DraII & RGGNCCY & 6 & five_prime & 1 & 72 \\
\hline MfeI & CAATTG & 6 & five_prime & 1 & 702 \\
\hline $\mathrm{NcoI}$ & CCATGG & 6 & five_prime & 1 & 1046 \\
\hline NheI & GCTAGC & 6 & five_prime & 1 & 526 \\
\hline NotI & GCGGCCGC & 8 & five_prime & 1 & 1115 \\
\hline PfoI & TCCNGGA & 6 & five_prime & 1 & 978 \\
\hline Tth111I & GACNNNGTC & 6 & five_prime & 1 & 898 \\
\hline XhoII & RGATCY & 6 & five_prime & 1 & 305 \\
\hline BfiI & ACTGGG & 6 & three_prime & 1 & 406 \\
\hline BglI & GCCNNNNNGGC & 6 & three_prime & 1 & 1123 \\
\hline BseRI & GAGGAG & 6 & three_prime & 1 & 316 \\
\hline BsgI & GTGCAG & 6 & three_prime & 1 & 872 \\
\hline BsrDI & GCAATG & 6 & three_prime & 1 & 933 \\
\hline BstXI & CCANNNNNNTGG & 6 & three_prime & 1 & 1188 \\
\hline HaeII & RGCGCY & 6 & three_prime & 1 & 455 \\
\hline MmeI & TCCRAC & 6 & three_prime & 1 & 1037 \\
\hline NmeAIII & GCCGAG & 6 & three_prime & 1 & 997 \\
\hline TspDTI & ATGAA & 5 & three_prime & 1 & 302 \\
\hline AflIII & ACRYGT & 6 & five_prime & 2 & 558,585 \\
\hline Cfr10I & RCCGGY & 6 & five_prime & 2 & 487,1171 \\
\hline PleI & GAGTC & 5 & five_prime & 2 & 159,770 \\
\hline SfaNI & GCATC & 5 & five_prime & 2 & 500,852 \\
\hline StyI & CCWWGG & 6 & five_prime & 2 & 388,1046 \\
\hline TfiI & GAWTC & 5 & five_prime & 2 & 667,732 \\
\hline BaeI & ACNNNNGTAYC & 7 & three_prime & 2 & 353,386 \\
\hline BciVI & GTATCC & 6 & three_prime & 2 & 477,769 \\
\hline BseMII & CTCAG & 5 & three_prime & 2 & 24,1118 \\
\hline Hрy99I & CGWCG & 5 & three_prime & 2 & 217,898 \\
\hline KpnI & GGTACC & 6 & three_prime & 2 & 357,467 \\
\hline NspI & RCATGY & 6 & three_prime & 2 & 562,589 \\
\hline PstI & CTGCAG & 6 & three_prime & 2 & 51,549 \\
\hline TaqII & GACCGA & 6 & three_prime & 2 & 672,698 \\
\hline TspRI & CASTG & 5 & three_prime & 2 & 63,909 \\
\hline TstI & CACNNNNNNTCC & 6 & three_prime & 2 & 628,660 \\
\hline MslI & CAYNNNNRTG & 6 & blunt & 3 & $320,416,563$ \\
\hline CfrI & YGGCCR & 6 & five_prime & 3 & $710,1049,1115$ \\
\hline FauI & CCCGC & 5 & five_prime & 3 & $32,234,267$ \\
\hline
\end{tabular}


Samia Abd Allah Abdal-Aziz and Safaa M. Ali / OnLine Journal of Biological Sciences 2021, 21 (1): 69.119 DOI: 10.3844/ojbsci.2021.69.119

\begin{tabular}{|c|c|c|c|c|c|}
\hline FokI & GGATG & 5 & five_prime & 3 & $73,82,706$ \\
\hline TatI & WGTACW & 6 & five_prime & 3 & $107,205,825$ \\
\hline BsrI & ACTGG & 5 & three_prime & 3 & $401,1145,1193$ \\
\hline SduI & GDGCHC & 6 & three_prime & 3 & $179,387,1133$ \\
\hline TsoI & TARCCA & 6 & three_prime & 3 & $487,1093,1132$ \\
\hline AvaII & GGWCC & 5 & five_prime & 4 & $72,596,737,956$ \\
\hline BsmI & GAATGC & 6 & three_prime & 4 & $4,17,813,1158$ \\
\hline Tsp45I & GTSAC & 5 & five_prime & 5 & $114,574,900,1062,1158$ \\
\hline AgsI & TTSAA & 5 & three_prime & 5 & $123,238,702,792,947$ \\
\hline HphI & GGTGA & 5 & three_prime & 5 & $565,628,676,1152,1237$ \\
\hline TspGWI & ACGGA & 5 & three_prime & 5 & $187,636,885,948,1214$ \\
\hline BbvI & GCAGC & 5 & five_prime & 6 & $14,200,252,531,760,877$ \\
\hline TseI & GCWGC & 5 & five_prime & 6 & $27,188,265,544,748,865$ \\
\hline BsaXI & ACNNNNNCTCC & 6 & three_prime & 6 & $5,35,974,1004,1018,1048$ \\
\hline Hin4I & GAYNNNNNVTC & 6 & three_prime & 6 & $5,37,717,749,974,1006$ \\
\hline BccI & CCATC & 5 & five_prime & 7 & $94,103,559,688,1039,1080,1228$ \\
\hline
\end{tabular}

Table 26: Restriction digestion of protease gene from XM_026751684.1 Aspergillus mulundensis

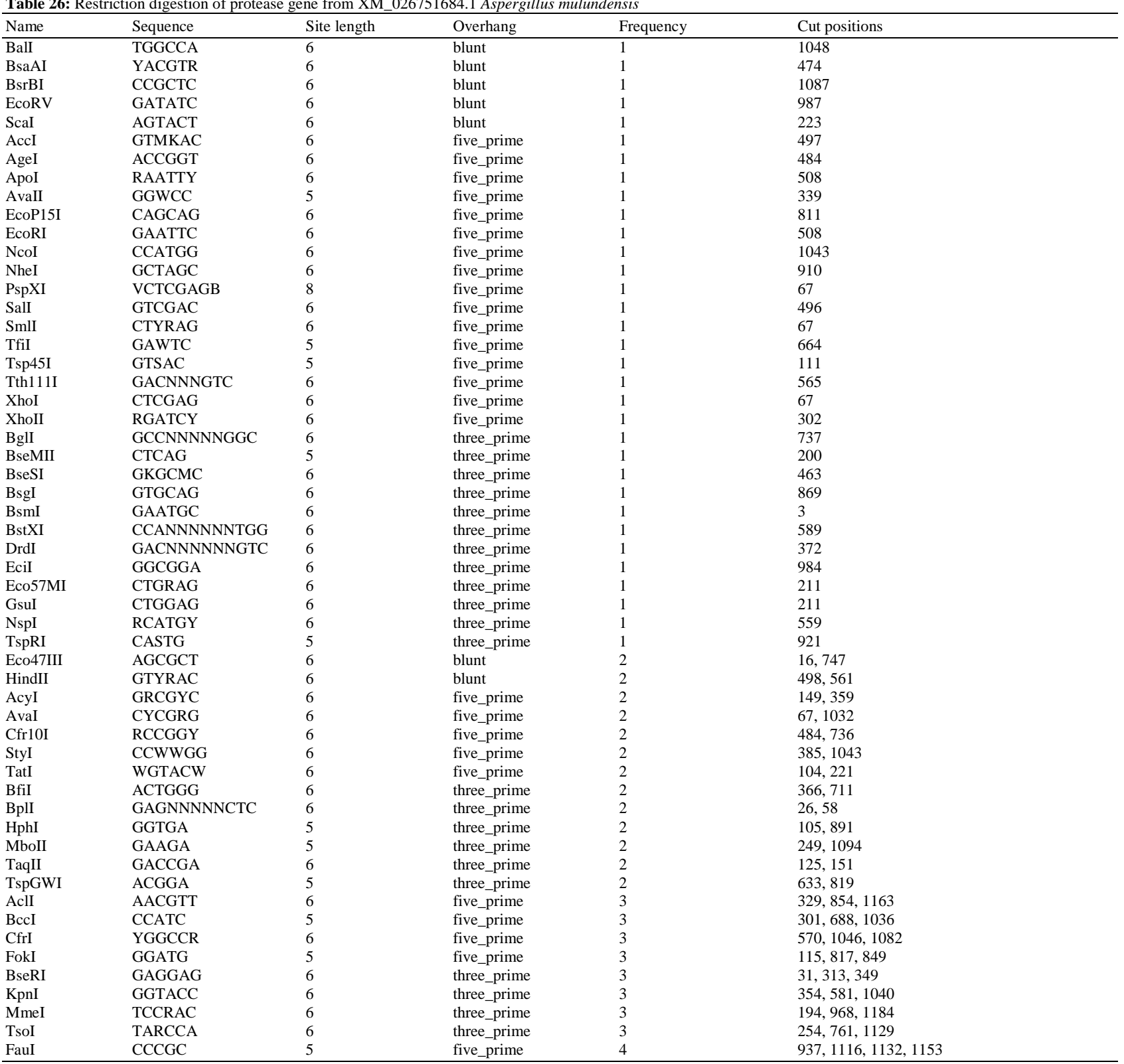


Samia Abd Allah Abdal-Aziz and Safaa M. Ali / OnLine Journal of Biological Sciences 2021, 21 (1): 69.119 DOI: 10.3844/ojbsci.2021.69.119

\begin{tabular}{|c|c|c|c|c|c|}
\hline BaeI & ACNNNNGTAYC & 7 & three_prime & 4 & $471,504,569,602$ \\
\hline $\mathrm{BcgI}$ & CGANNNNNNTGC & 6 & three_prime & 4 & $942,952,976,986$ \\
\hline BsrI & ACTGG & 5 & three_prime & 4 & $219,372,417,706$ \\
\hline BbvI & GCAGC & 5 & five_prime & 5 & $683,693,825,894,1184$ \\
\hline HgaI & GACGC & 5 & five_prime & 5 & $157,184,367,555,923$ \\
\hline TseI & GCWGC & 5 & five_prime & 5 & $671,706,838,907,1172$ \\
\hline AgsI & TTSAA & 5 & three_prime & 5 & $12,120,699,789,1093$ \\
\hline EcoRII & CCWGG & 5 & five_prime & 6 & $188,656,791,975,997,1079$ \\
\hline SfaNI & GCATC & 5 & five_prime & 6 & $137,276,795,849,871,1136$ \\
\hline BsaXI & ACNNNNNCTCC & 6 & three_prime & 6 & $208,238,625,655,1141,1171$ \\
\hline Hin4I & GAYNNNNNVTC & 6 & three_prime & 6 & $208,240,850,882,976,1008$ \\
\hline TauI & GCSGC & 5 & three_prime & 6 & $203,521,734,1087,1114,1172$ \\
\hline Hpy99I & CGWCG & 5 & three_prime & 10 & $80,151,200,286,571,694,802,961,964,1066$ \\
\hline
\end{tabular}

Table 27: Restriction digestion of protease gene from XM_025625888.1 Aspergillus neoniger

\begin{tabular}{|c|c|c|c|c|c|}
\hline Name & Sequence & Site length & Overhang & Frequency & Cut positions \\
\hline BalI & TGGCCA & 6 & blunt & 1 & 1063 \\
\hline BsaAI & YACGTR & 6 & blunt & 1 & 345 \\
\hline Eco47III & AGCGCT & 6 & blunt & 1 & 465 \\
\hline HindII & GTYRAC & 6 & blunt & 1 & 978 \\
\hline NaeI & GCCGGC & 6 & blunt & 1 & 243 \\
\hline OliI & CACNNNNGTG & 6 & blunt & 1 & 428 \\
\hline PshAI & GACNNNNGTC & 6 & blunt & 1 & 694 \\
\hline SmaI & CCCGGG & 6 & blunt & 1 & 113 \\
\hline AsuII & TTCGAA & 6 & five_prime & 1 & 637 \\
\hline AvaI & CYCGRG & 6 & five_prime & 1 & 111 \\
\hline BsmAI & GTCTC & 5 & five_prime & 1 & 1143 \\
\hline BspMI & ACCTGC & 6 & five_prime & 1 & 72 \\
\hline DraII & RGGNCCY & 6 & five_prime & 1 & 84 \\
\hline EcoRII & CCWGG & 5 & five_prime & 1 & 1112 \\
\hline MfeI & CAATTG & 6 & five_prime & 1 & 714 \\
\hline NcoI & CCATGG & 6 & five_prime & 1 & 1058 \\
\hline NotI & GCGGCCGC & 8 & five_prime & 1 & 1127 \\
\hline PfoI & TCCNGGA & 6 & five_prime & 1 & 990 \\
\hline Tth111I & GACNNNGTC & 6 & five_prime & 1 & 910 \\
\hline XhoII & RGATCY & 6 & five_prime & 1 & 317 \\
\hline BfiI & ACTGGG & 6 & three_prime & 1 & 418 \\
\hline BglI & GCCNNNNNGGC & 6 & three_prime & 1 & 1135 \\
\hline BseRI & GAGGAG & 6 & three_prime & 1 & 328 \\
\hline BsgI & GTGCAG & 6 & three_prime & 1 & 884 \\
\hline BsrDI & GCAATG & 6 & three_prime & 1 & 945 \\
\hline BstXI & CCANNNNNNTGG & 6 & three_prime & 1 & 1200 \\
\hline HaeII & RGCGCY & 6 & three_prime & 1 & 467 \\
\hline MmeI & TCCRAC & 6 & three_prime & 1 & 1049 \\
\hline NmeAIII & GCCGAG & 6 & three_prime & 1 & 1009 \\
\hline TspDTI & ATGAA & 5 & three_prime & 1 & 314 \\
\hline AflIII & ACRYGT & 6 & five_prime & 2 & 570,597 \\
\hline BseYI & CCCAGC & 6 & five_prime & 2 & 277,1122 \\
\hline PleI & GAGTC & 5 & five_prime & 2 & 171,782 \\
\hline StyI & CCWWGG & 6 & five_prime & 2 & 400,1058 \\
\hline TfiI & GAWTC & 5 & five_prime & 2 & 679,744 \\
\hline AlfI & GCANNNNNNTGC & 6 & three_prime & 2 & 412,446 \\
\hline BseMII & CTCAG & 5 & three_prime & 2 & 36,1130 \\
\hline Нpy99I & CGWCG & 5 & three_prime & 2 & 229,910 \\
\hline KpnI & GGTACC & 6 & three_prime & 2 & 369,479 \\
\hline NspI & RCATGY & 6 & three_prime & 2 & 574,601 \\
\hline PstI & CTGCAG & 6 & three_prime & 2 & 63,561 \\
\hline TaqII & GACCGA & 6 & three_prime & 2 & 684,710 \\
\hline TspRI & CASTG & 5 & three_prime & 2 & 75,921 \\
\hline TstI & CACNNNNNNTCC & 6 & three_prime & 2 & 640,672 \\
\hline MslI & CAYNNNNRTG & 6 & blunt & 3 & $332,428,575$ \\
\hline Cfr10I & RCCGGY & 6 & five_prime & 3 & $241,499,1183$ \\
\hline CfrI & YGGCCR & 6 & five_prime & 3 & $722,1061,1127$ \\
\hline FauI & CCCGC & 5 & five_prime & 3 & $44,246,279$ \\
\hline FokI & GGATG & 5 & five_prime & 3 & $85,94,718$ \\
\hline SfaNI & GCATC & 5 & five_prime & 3 & $254,512,864$ \\
\hline TatI & WGTACW & 6 & five_prime & 3 & $119,217,837$ \\
\hline Tsp45I & GTSAC & 5 & five_prime & 3 & $586,912,1074$ \\
\hline BsrI & ACTGG & 5 & three_prime & 3 & $413,1157,1205$ \\
\hline SacI & GAGCTC & 6 & three_prime & 3 & $191,399,1145$ \\
\hline
\end{tabular}


Samia Abd Allah Abdal-Aziz and Safaa M. Ali / OnLine Journal of Biological Sciences 2021, 21 (1): 69.119 DOI: 10.3844/ojbsci.2021.69.119

$\begin{array}{llllll}\text { Table 27: Countiue } & & & & & \\ \text { SduI } & \text { GDGCHC } & 6 & \text { three_prime } & 3 & 191,399,1145 \\ \text { TsoI } & \text { TARCCA } & 6 & \text { three_prime } & 3 & 49,1105,1144 \\ \text { AvaII } & \text { GGWCC } & 5 & \text { five_prime } & 4 & 84,608,749,968 \\ \text { AgsI } & \text { TTSAA } & 5 & \text { three_prime } & 4 & 135,714,804,959 \\ \text { BsmI } & \text { GAATGC } & 6 & \text { three_prime } & 4 & 16,29,825,1170 \\ \text { BbvI } & \text { GCAGC } & 5 & \text { five_prime } & 5 & 26,212,543,772,889 \\ \text { HgaI } & \text { GACGC } & 5 & \text { five_prime } & 5 & 35,206,422,916,938 \\ \text { TseI } & \text { GCWGC } & 5 & \text { five_prime } & 5 & 39,200,556,760,877 \\ \text { HphI } & \text { GGTGA } & 5 & \text { three_prime } & 5 & 57,640,688,1164,1249 \\ \text { TspGWI } & \text { ACGGA } & 5 & \text { three_prime } & 5 & 199,648,897,960,1226 \\ \text { BsaXI } & \text { ACNNNNNCTCC } & 6 & \text { three_prime } & 6 & 17,47,986,1016,1030,1060 \\ \text { Hin4I } & \text { GAYNNNNNVTC } & 6 & \text { three_prime } & 6 & 7 \\ \text { BccI } & \text { CCATC } & 5 & \text { five_prime } & 7,79,729,761,986,1018 \\ \text { TauI } & \text { GCSGC } & 5 & \text { three_prime } & 8 & 106,115,571,700,1051,1092,1240 \\ \end{array}$

Table 28: Restriction digestion of protease gene from L19059.1 Aspergillus niger

\begin{tabular}{|c|c|c|c|c|c|}
\hline Name & Sequence & Site length & Overhang & Frequency & Cut positions \\
\hline$\overline{\text { BsaAI }}$ & YACGTR & 6 & blunt & 1 & 1165 \\
\hline BsaBI & GATNNNNATC & 6 & blunt & 1 & 669 \\
\hline Eco47III & AGCGCT & 6 & blunt & 1 & 1333 \\
\hline EcoRV & GATATC & 6 & blunt & 1 & 1600 \\
\hline HpaI & GTTAAC & 6 & blunt & 1 & 1683 \\
\hline PshAI & GACNNNNGTC & 6 & blunt & 1 & 1562 \\
\hline ScaI & AGTACT & 6 & blunt & 1 & 1974 \\
\hline AarI & CACCTGC & 7 & five_prime & 1 & 1684 \\
\hline AcyI & GRCGYC & 6 & five_prime & 1 & 1878 \\
\hline AflIII & ACRYGT & 6 & five_prime & 1 & 1465 \\
\hline ApoI & RAATTY & 6 & five_prime & 1 & 1818 \\
\hline AsuII & TTCGAA & 6 & five_prime & 1 & 1505 \\
\hline AvrII & CCTAGG & 6 & five_prime & 1 & 2790 \\
\hline BamHI & GGATCC & 6 & five_prime & 1 & 2988 \\
\hline BspMI & ACCTGC & 6 & five_prime & 1 & 1684 \\
\hline BtgZI & GCGATG & 6 & five_prime & 1 & 2226 \\
\hline CfrI & YGGCCR & 6 & five_prime & 1 & 2001 \\
\hline ClaI & ATCGAT & 6 & five_prime & 1 & 427 \\
\hline NcoI & CCATGG & 6 & five_prime & 1 & 2028 \\
\hline NheI & GCTAGC & 6 & five_prime & 1 & 1406 \\
\hline PspXI & VCTCGAGB & 8 & five_prime & 1 & 2863 \\
\hline RsrII & CGGWCCG & 7 & five_prime & 1 & 2496 \\
\hline SpeI & ACTAGT & 6 & five_prime & 1 & 2284 \\
\hline $\mathrm{XbaI}$ & TCTAGA & 6 & five_prime & 1 & 2645 \\
\hline XhoI & CTCGAG & 6 & five_prime & 1 & 2863 \\
\hline AlwNI & CAGNNNCTG & 6 & three_prime & 1 & 2099 \\
\hline BciVI & GTATCC & 6 & three_prime & 1 & 1177 \\
\hline BseRI & GAGGAG & 6 & three_prime & 1 & 1145 \\
\hline BstXI & CCANNNNNNTGG & 6 & three_prime & 1 & 2170 \\
\hline BtsI & GCAGTG & 6 & three_prime & 1 & 365 \\
\hline Eco57I & CTGAAG & 6 & three_prime & 1 & 270 \\
\hline GsuI & CTGGAG & 6 & three_prime & 1 & 2825 \\
\hline NmeAIII & GCCGAG & 6 & three_prime & 1 & 1979 \\
\hline BtrI & CACGTC & 6 & blunt & 2 & 2025,2487 \\
\hline HindII & GTYRAC & 6 & blunt & 2 & 1683,1948 \\
\hline OliI & CACNNNNGTG & 6 & blunt & 2 & 1296,2147 \\
\hline Bsp1407I & TGTACA & 6 & five_prime & 2 & 313,1198 \\
\hline DraII & RGGNCCY & 6 & five_prime & 2 & 901,2080 \\
\hline EcoNI & CCTNNNNNAGG & 6 & five_prime & 2 & 161,315 \\
\hline MfeI & CAATTG & 6 & five_prime & 2 & 191,1582 \\
\hline PpuMI & RGGWCCY & 7 & five_prime & 2 & 901,2080 \\
\hline Tth111I & GACNNNGTC & 6 & five_prime & 2 & 28,652 \\
\hline AgsI & TTSAA & 5 & three_prime & 2 & 952,1582 \\
\hline AlfI & GCANNNNNNTGC & 6 & three_prime & 2 & 1826,1860 \\
\hline BaeI & ACNNNNGTAYC & 7 & three_prime & 2 & 1233,1266 \\
\hline BfiI & ACTGGG & 6 & three_prime & 2 & 577,1286 \\
\hline BplI & GAGNNNNNCTC & 6 & three_prime & 2 & 1273,1305 \\
\hline BsgI & GTGCAG & 6 & three_prime & 2 & 1854,2435 \\
\hline Eco57MI & CTGRAG & 6 & three_prime & 2 & 270,2825 \\
\hline FalI & AAGNNNNNCTT & 6 & three_prime & 2 & 1035,1067 \\
\hline HaeII & RGCGCY & 6 & three_prime & 2 & 1335,2951 \\
\hline Hin4I & GAYNNNNNVTC & 6 & three_prime & 2 & 2405,2437 \\
\hline Нру99I & CGWCG & 5 & three_prime & 2 & 1880,2488 \\
\hline KpnI & GGTACC & 6 & three_prime & 2 & 1237,2494 \\
\hline PstI & CTGCAG & 6 & three_prime & 2 & 880,1429 \\
\hline
\end{tabular}


Samia Abd Allah Abdal-Aziz and Safaa M. Ali / OnLine Journal of Biological Sciences 2021, 21 (1): 69.119 DOI: 10.3844/ojbsci.2021.69.119

\begin{tabular}{|c|c|c|c|c|c|}
\hline SphI & GCATGC & 6 & three_prime & 2 & 534,555 \\
\hline SmaI & CCCGGG & 6 & blunt & 3 & $930,1962,2903$ \\
\hline Cfr10I & RCCGGY & 6 & five_prime & 3 & $67,1988,2702$ \\
\hline PleI & GAGTC & 5 & five_prime & 3 & $428,988,1650$ \\
\hline SmlI & CTYRAG & 6 & five_prime & 3 & $2114,2379,2863$ \\
\hline StyI & CCWWGG & 6 & five_prime & 3 & $1268,2028,2790$ \\
\hline TfiI & GAWTC & 5 & five_prime & 3 & $1547,2642,2878$ \\
\hline MmeI & TCCRAC & 6 & three_prime & 3 & $935,1432,2615$ \\
\hline BseYI & CCCAGC & 6 & five_prime & 4 & $1094,1334,2092,2396$ \\
\hline EcoP15I & CAGCAG & 6 & five_prime & 4 & $445,829,1166,1765$ \\
\hline XhoII & RGATCY & 6 & five_prime & 4 & $747,1134,1669,2988$ \\
\hline ArsI & GACNNNNNNTTYG & 7 & three_prime & 4 & $965,997,1816,1848$ \\
\hline BdaI & TGANNNNNNTCA & 6 & three_prime & 4 & $394,428,653,687$ \\
\hline BseMII & CTCAG & 5 & three_prime & 4 & $156,475,607,2789$ \\
\hline MboII & GAAGA & 5 & three_prime & 4 & $263,605,1055,2159$ \\
\hline NspI & RCATGY & 6 & three_prime & 4 & $534,555,1469,2458$ \\
\hline TspGWI & ACGGA & 5 & three_prime & 4 & $1016,1516,1867,2719$ \\
\hline TstI & CACNNNNNNTCC & 6 & three_prime & 4 & $1468,1500,1508,1540$ \\
\hline AvaI & CYCGRG & 6 & five_prime & 5 & $928,1960,2409,2863,2901$ \\
\hline HgaI & GACGC & 5 & five_prime & 5 & $605,1290,1886,1908,2947$ \\
\hline Tsp45I & GTSAC & 5 & five_prime & 5 & $603,688,2044,2140,2705$ \\
\hline BsmI & GAATGC & 6 & three_prime & 5 & $103,776,846,1740,2140$ \\
\hline BsrI & ACTGG & 5 & three_prime & 5 & $583,1281,1372,1620,2808$ \\
\hline TsoI & TARCCA & 6 & three_prime & 5 & $474,1163,1367,2021,2114$ \\
\hline TspDTI & ATGAA & 5 & three_prime & 5 & $360,419,2319,2655,2713$ \\
\hline TspRI & CASTG & 5 & three_prime & 5 & $365,462,892,1372,2629$ \\
\hline MslI & CAYNNNNRTG & 6 & blunt & 6 & $1149,1155,1296,1443,2027,2147$ \\
\hline BsmAI & GTCTC & 5 & five_prime & 6 & $232,729,987,2406,2801,2930$ \\
\hline TaqII & GACCGA & 6 & three_prime & 6 & $1552,1578,2097,2123,2487,2513$ \\
\hline FauI & CCCGC & 5 & five_prime & 8 & $158,315,666,861,1063,1096,2070,2146$ \\
\hline SfaNI & GCATC & 5 & five_prime & 8 & $136,189,244,549,1293,1380,1680,1834$ \\
\hline TatI & WGTACW & 6 & five_prime & 8 & $121,313,936,1034,1198,1972,2601,2767$ \\
\hline BsaXI & ACNNNNNCTCC & 6 & three_prime & 8 & $834,864,1956,1986,2000,2030,2532,2562$ \\
\hline FokI & GGATG & 5 & five_prime & 9 & $288,509,894,902,911,1586,2414,2449,2772$ \\
\hline HphI & GGTGA & 5 & three_prime & 10 & $125,174,290,694,1283,1445,1508,1556,2134,2717$ \\
\hline TseI & GCWGC & 5 & five_prime & 11 & $58,491,856,1017,1424,1589,1847,2099,2468,2582,2950$ \\
\hline SduI & GDGCHC & 6 & three_prime & 11 & $95,462,1008,1267,1440,1757,2115,2550,2789,2799,2970$ \\
\hline AvaII & GGWCC & 5 & five_prime & 13 & $44,65,209,654,704,901,1476,1617,1986,2080,2496,2520,2742$ \\
\hline BccI & CCATC & 5 & five_prime & 14 & $261,309,472,530,616,923,932,1439,1487,1568,2062,2210,2620,2754$ \\
\hline
\end{tabular}

Table 29: Restriction digestion of protease gene from XM_025719167.1 Aspergillus ibericus

\begin{tabular}{|c|c|c|c|c|c|}
\hline Name & Sequence & Site length & Overhang & Frequency & Cut positions \\
\hline$\overline{\text { BalI }}$ & TGGCCA & 6 & blunt & 1 & 1282 \\
\hline BsaBI & GATNNNNATC & 6 & blunt & 1 & 356 \\
\hline NaeI & GCCGGC & 6 & blunt & 1 & 414 \\
\hline NruI & TCGCGA & 6 & blunt & 1 & 998 \\
\hline SmaI & CCCGGG & 6 & blunt & 1 & 1251 \\
\hline SspI & AATATT & 6 & blunt & 1 & 1128 \\
\hline AccI & GTMKAC & 6 & five_prime & 1 & 1103 \\
\hline AvaI & CYCGRG & 6 & five_prime & 1 & 1249 \\
\hline BsmAI & GTCTC & 5 & five_prime & 1 & 1010 \\
\hline ClaI & ATCGAT & 6 & five_prime & 1 & 1183 \\
\hline EcoP15I & CAGCAG & 6 & five_prime & 1 & 157 \\
\hline Esp3I & CGTCTC & 6 & five_prime & 1 & 1010 \\
\hline FokI & GGATG & 5 & five_prime & 1 & 844 \\
\hline NdeI & CATATG & 6 & five_prime & 1 & 753 \\
\hline SalI & GTCGAC & 6 & five_prime & 1 & 1102 \\
\hline SexAI & ACCWGGT & 7 & five_prime & 1 & 482 \\
\hline SmlI & CTYRAG & 6 & five_prime & 1 & 1270 \\
\hline StyI & CCWWGG & 6 & five_prime & 1 & 526 \\
\hline AatII & GACGTC & 6 & three_prime & 1 & 820 \\
\hline BseMII & CTCAG & 5 & three_prime & 1 & 31 \\
\hline BseSI & GKGCMC & 6 & three_prime & 1 & 604 \\
\hline BsmI & GAATGC & 6 & three_prime & 1 & 774 \\
\hline BsrDI & GCAATG & 6 & three_prime & 1 & 1071 \\
\hline DrdI & GACNNNNNNGTC & 6 & three_prime & 1 & 505 \\
\hline HaeII & RGCGCY & 6 & three_prime & 1 & 593 \\
\hline SacI & GAGCTC & 6 & three_prime & 1 & 814 \\
\hline TsoI & TARCCA & 6 & three_prime & 1 & 113 \\
\hline TspGWI & ACGGA & 5 & three_prime & 1 & 774 \\
\hline
\end{tabular}


Samia Abd Allah Abdal-Aziz and Safaa M. Ali / OnLine Journal of Biological Sciences 2021, 21 (1): 69.119 DOI: 10.3844/ojbsci.2021.69.119

\begin{tabular}{|c|c|c|c|c|c|}
\hline TspRI & CASTG & 5 & three_prime & 1 & 573 \\
\hline HindII & GTYRAC & 6 & blunt & 2 & 702,1104 \\
\hline PshAI & GACNNNNGTC & 6 & blunt & 2 & 41,820 \\
\hline BglII & AGATCT & 6 & five_prime & 2 & 357,776 \\
\hline BtgZI & GCGATG & 6 & five_prime & 2 & 1013,1388 \\
\hline CfrI & YGGCCR & 6 & five_prime & 2 & 657,1280 \\
\hline DraII & RGGNCCY & 6 & five_prime & 2 & 62,531 \\
\hline HgaI & GACGC & 5 & five_prime & 2 & 992,1340 \\
\hline SgrAI & CRCCGGYG & 8 & five_prime & 2 & 592,685 \\
\hline BciVI & GTATCC & 6 & three_prime & 2 & 615,640 \\
\hline BfiI & ACTGGG & 6 & three_prime & 2 & 16,852 \\
\hline BglI & GCCNNNNNGGC & 6 & three_prime & 2 & 335,670 \\
\hline BseRI & GAGGAG & 6 & three_prime & 2 & 454,661 \\
\hline Hin4I & GAYNNNNNVTC & 6 & three_prime & 2 & 1176,1208 \\
\hline KpnI & GGTACC & 6 & three_prime & 2 & 176,495 \\
\hline NspI & RCATGY & 6 & three_prime & 2 & 700,727 \\
\hline $\mathrm{XcmI}$ & CCANNNNNNNNNTGG & 6 & three_prime & 2 & 235,649 \\
\hline MslI & CAYNNNNRTG & 6 & blunt & 3 & $458,895,1183$ \\
\hline Cfr10I & RCCGGY & 6 & five_prime & 3 & $412,592,685$ \\
\hline PleI & GAGTC & 5 & five_prime & 3 & $50,908,1414$ \\
\hline BsrI & ACTGG & 5 & three_prime & 3 & $11,207,847$ \\
\hline MmeI & TCCRAC & 6 & three_prime & 3 & $348,690,1175$ \\
\hline SduI & GDGCHC & 6 & three_prime & 3 & $525,604,814$ \\
\hline TauI & GCSGC & 5 & three_prime & 3 & $685,850,1258$ \\
\hline TspDTI & ATGAA & 5 & three_prime & 3 & $368,440,1391$ \\
\hline XholI & RGATCY & 6 & five_prime & 4 & $224,357,443,776$ \\
\hline $\mathrm{BcgI}$ & CGANNNNNNTGC & 6 & three_prime & 4 & $1064,1093,1098,1127$ \\
\hline AvaII & GGWCC & 5 & five_prime & 5 & $480,734,1010,1093,1148$ \\
\hline Tsp45I & GTSAC & 5 & five_prime & 5 & $507,1038,1200,1296,1366$ \\
\hline AgsI & TTSAA & 5 & three_prime & 5 & $150,261,289,840,930$ \\
\hline HphI & GGTGA & 5 & three_prime & 5 & $26,208,607,1290,1378$ \\
\hline BbvI & GCAGC & 5 & five_prime & 6 & $152,319,338,873,991,1035$ \\
\hline EcoRII & CCWGG & 5 & five_prime & 6 & $64,237,482,668,1116,1138$ \\
\hline TfiI & GAWTC & 5 & five_prime & 6 & $289,371,805,836,993,1439$ \\
\hline TseI & GCWGC & 5 & five_prime & 6 & $165,307,326,886,979,1048$ \\
\hline SfaNI & GCATC & 5 & five_prime & 8 & $267,551,568,990,1023,1030,1084,1374$ \\
\hline BccI & CCATC & 5 & five_prime & 10 & $85,132,442,745,826,898,1177,1218,1418,1427$ \\
\hline
\end{tabular}

Table 30: Restriction digestion of protease gene from XM_025536473.1 Aspergillus eucalypticola

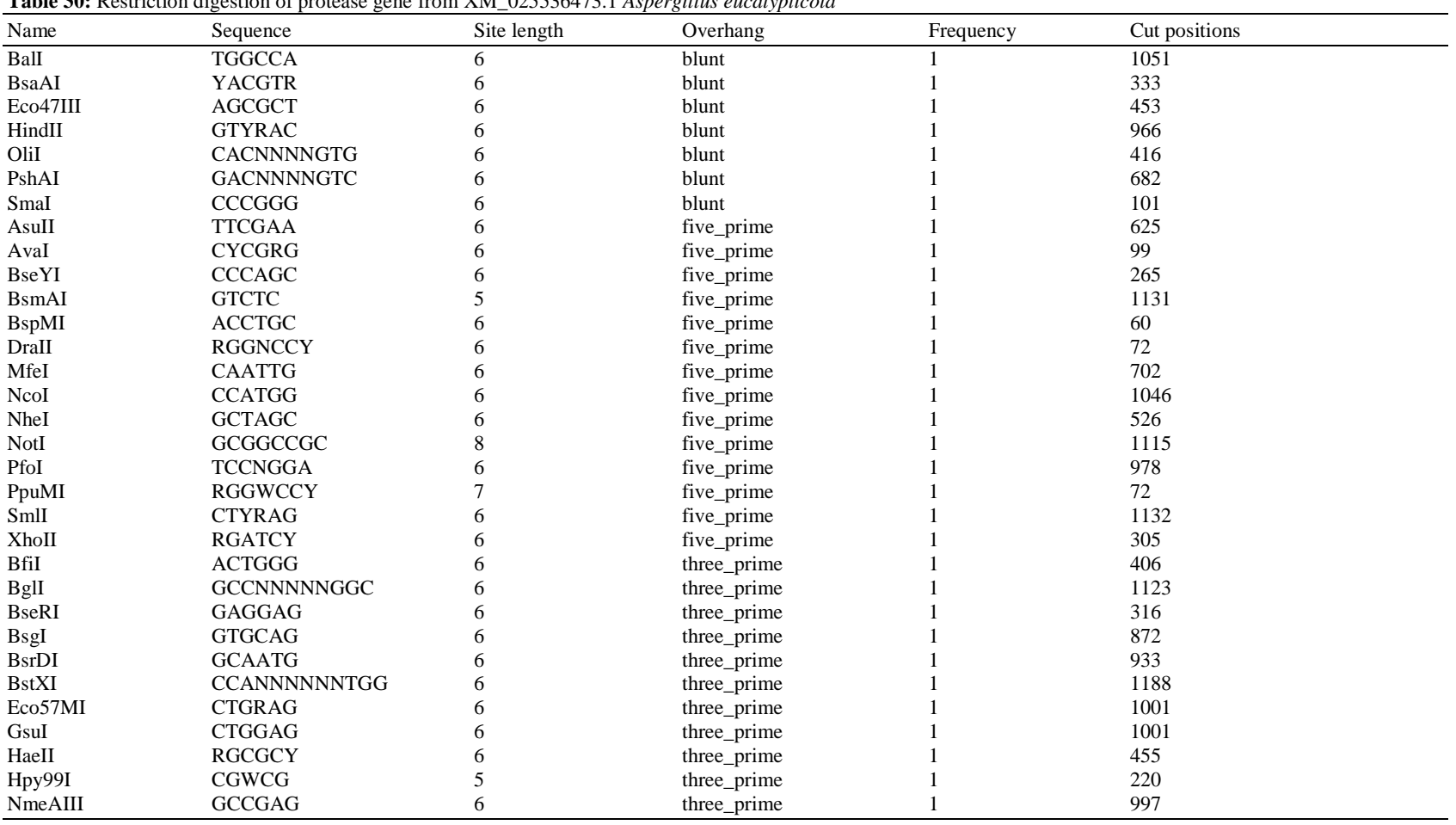


Samia Abd Allah Abdal-Aziz and Safaa M. Ali / OnLine Journal of Biological Sciences 2021, 21 (1): 69.119 DOI: 10.3844/ojbsci.2021.69.119

\begin{tabular}{|c|c|c|c|c|c|}
\hline NspI & RCATGY & 6 & three_prime & 1 & 589 \\
\hline TspDTI & ATGAA & 5 & three_prime & 1 & 302 \\
\hline NaeI & GCCGGC & 6 & blunt & 2 & 129,231 \\
\hline CfrI & YGGCCR & 6 & five_prime & 2 & 1049,1115 \\
\hline EcoRII & CCWGG & 5 & five_prime & 2 & 978,1100 \\
\hline HgaI & GACGC & 5 & five_prime & 2 & 194,410 \\
\hline PleI & GAGTC & 5 & five_prime & 2 & 159,770 \\
\hline TfiI & GAWTC & 5 & five_prime & 2 & 667,732 \\
\hline AlfI & GCANNNNNNTGC & 6 & three_prime & 2 & 400,434 \\
\hline BaeI & ACNNNNGTAYC & 7 & three_prime & 2 & 353,386 \\
\hline BciVI & GTATCC & 6 & three_prime & 2 & 477,769 \\
\hline KpnI & GGTACC & 6 & three_prime & 2 & 357,467 \\
\hline PstI & CTGCAG & 6 & three_prime & 2 & 51,549 \\
\hline TaqII & GACCGA & 6 & three_prime & 2 & 672,698 \\
\hline TspRI & CASTG & 5 & three_prime & 2 & 63,909 \\
\hline TstI & CACNNNNNNTCC & 6 & three_prime & 2 & 628,660 \\
\hline FauI & CCCGC & 5 & five_prime & 3 & $32,234,267$ \\
\hline TatI & WGTACW & 6 & five_prime & 3 & $107,205,825$ \\
\hline Tsp45I & GTSAC & 5 & five_prime & 3 & $900,1062,1158$ \\
\hline BsmI & GAATGC & 6 & three_prime & 3 & $17,813,1158$ \\
\hline BsrI & ACTGG & 5 & three_prime & 3 & $401,1145,1193$ \\
\hline MmeI & TCCRAC & 6 & three_prime & 3 & $106,552,1037$ \\
\hline SacI & GAGCTC & 6 & three_prime & 3 & $179,387,1133$ \\
\hline SduI & GDGCHC & 6 & three_prime & 3 & $179,387,1133$ \\
\hline TsoI & TARCCA & 6 & three_prime & 3 & $487,1093,1132$ \\
\hline AvalI & GGWCC & 5 & five_prime & 4 & $72,596,737,956$ \\
\hline Cfr10I & RCCGGY & 6 & five_prime & 4 & $127,229,487,1171$ \\
\hline FokI & GGATG & 5 & five_prime & 4 & $65,73,82,706$ \\
\hline SfaNI & GCATC & 5 & five_prime & 4 & $242,500,852,885$ \\
\hline AgsI & TTSAA & 5 & three_prime & 4 & $123,702,792,947$ \\
\hline ArsI & GACNNNNNNTTYG & 7 & three_prime & 4 & $136,168,940,972$ \\
\hline Hin4I & GAYNNNNNVTC & 6 & three_prime & 4 & $717,749,974,1006$ \\
\hline HphI & GGTGA & 5 & three_prime & 4 & $565,628,676,1237$ \\
\hline TspGWI & ACGGA & 5 & three_prime & 4 & $187,636,885,948$ \\
\hline BbvI & GCAGC & 5 & five_prime & 5 & $14,531,721,760,877$ \\
\hline BccI & CCATC & 5 & five_prime & 6 & $94,103,559,688,1080,1228$ \\
\hline BsaXI & ACNNNNNCTCC & 6 & three_prime & 6 & $5,35,628,658,974,1004$ \\
\hline TauI & GCSGC & 5 & three_prime & 7 & $203,608,844,913,1117,1120,1211$ \\
\hline
\end{tabular}

Table 31: Restriction digestion of protease gene from XM_025662057.1 Aspergillus piperis

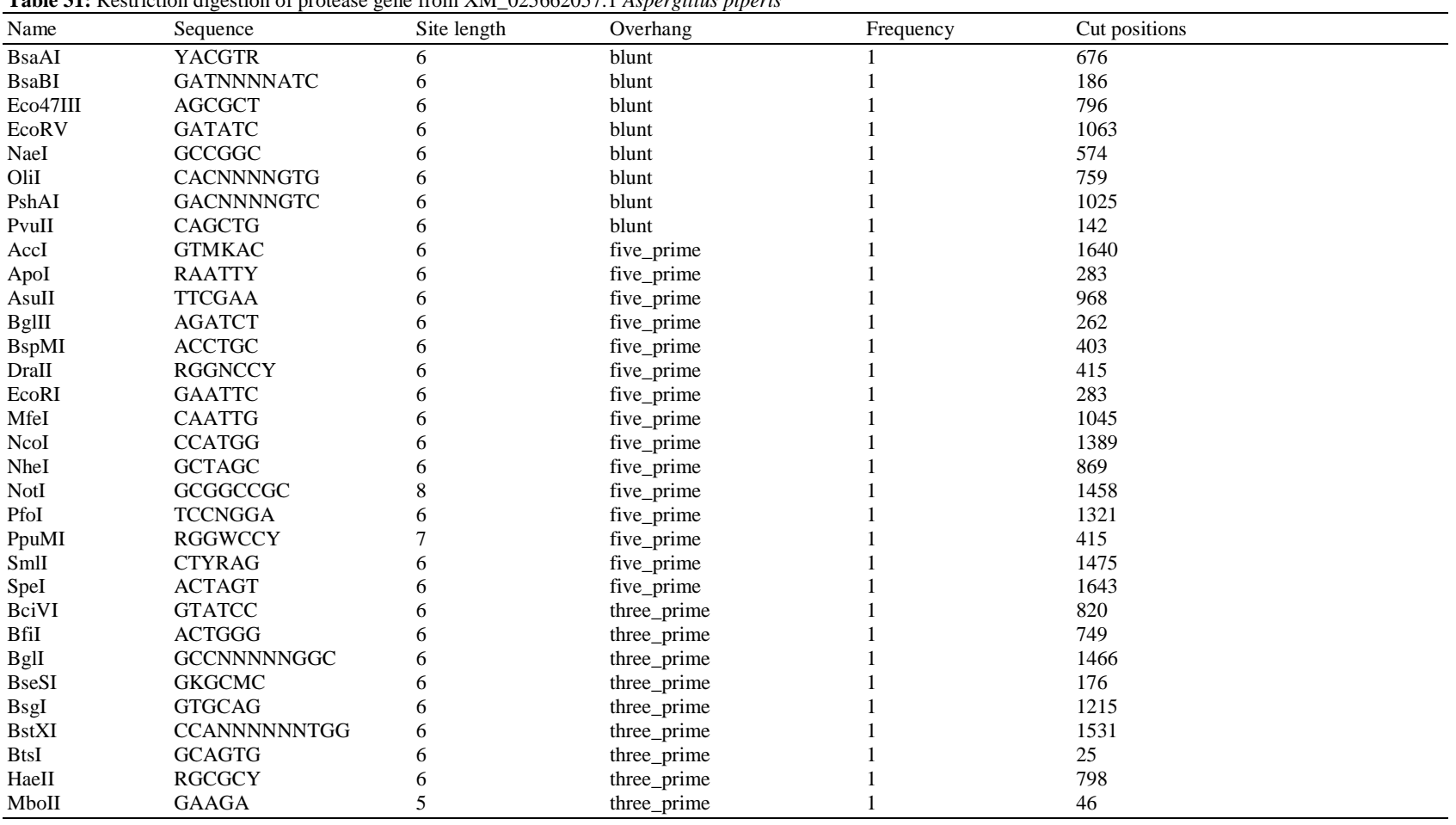


Samia Abd Allah Abdal-Aziz and Safaa M. Ali / OnLine Journal of Biological Sciences 2021, 21 (1): 69.119 DOI: 10.3844/ojbsci.2021.69.119

\begin{tabular}{|c|c|c|c|c|c|}
\hline NmeAIII & GCCGAG & 6 & three_prime & 1 & 1340 \\
\hline HindII & GTYRAC & 6 & blunt & 2 & 228,1309 \\
\hline AflIII & ACRYGT & 6 & five_prime & 2 & 901,928 \\
\hline BsmAI & GTCTC & 5 & five_prime & 2 & 245,1474 \\
\hline FauI & CCCGC & 5 & five_prime & 2 & 375,610 \\
\hline PleI & GAGTC & 5 & five_prime & 2 & 502,1113 \\
\hline StyI & CCWWGG & 6 & five_prime & 2 & 731,1389 \\
\hline AlfI & GCANNNNNNTGC & 6 & three_prime & 2 & 743,777 \\
\hline BaeI & ACNNNNGTAYC & 7 & three_prime & 2 & 696,729 \\
\hline BdaI & TGANNNNNNTCA & 6 & three_prime & 2 & 170,204 \\
\hline BseMII & CTCAG & 5 & three_prime & 2 & 367,1461 \\
\hline BseRI & GAGGAG & 6 & three_prime & 2 & 307,659 \\
\hline BsrDI & GCAATG & 6 & three_prime & 2 & 270,1276 \\
\hline Hpy99I & CGWCG & 5 & three_prime & 2 & 105,560 \\
\hline KpnI & GGTACC & 6 & three_prime & 2 & 700,810 \\
\hline NspI & RCATGY & 6 & three_prime & 2 & 905,932 \\
\hline TaqII & GACCGA & 6 & three_prime & 2 & 1015,1041 \\
\hline TsoI & TARCCA & 6 & three_prime & 2 & 830,1436 \\
\hline TspDTI & ATGAA & 5 & three_prime & 2 & 20,645 \\
\hline TstI & CACNNNNNNTCC & 6 & three_prime & 2 & 971,1003 \\
\hline BalI & TGGCCA & 6 & blunt & 3 & $146,1394,1487$ \\
\hline MsII & CAYNNNNRTG & 6 & blunt & 3 & $663,759,906$ \\
\hline AvaII & GGWCC & 5 & five_prime & 3 & $415,939,1080$ \\
\hline Cfr10I & RCCGGY & 6 & five_prime & 3 & $572,830,1514$ \\
\hline HgaI & GACGC & 5 & five_prime & 3 & $366,537,1269$ \\
\hline TfiI & GAWTC & 5 & five_prime & 3 & $93,1010,1075$ \\
\hline BsrI & ACTGG & 5 & three_prime & 3 & $744,1488,1536$ \\
\hline MmeI & TCCRAC & 6 & three_prime & 3 & $449,895,1380$ \\
\hline SacI & GAGCTC & 6 & three_prime & 3 & $522,730,1476$ \\
\hline TspRI & CASTG & 5 & three_prime & 3 & $25,406,1252$ \\
\hline EcoRII & CCWGG & 5 & five_prime & 4 & $41,442,873,1443$ \\
\hline FokI & GGATG & 5 & five_prime & 4 & $408,416,425,1049$ \\
\hline SfaNI & GCATC & 5 & five_prime & 4 & $585,843,1195,1584$ \\
\hline TatI & WGTACW & 6 & five_prime & 4 & $85,450,548,1168$ \\
\hline ArsI & GACNNNNNNTTYG & 7 & three_prime & 4 & $225,257,479,511$ \\
\hline CfrI & YGGCCR & 6 & five_prime & 5 & $144,949,1392,1458,1485$ \\
\hline Tsp45I & GTSAC & 5 & five_prime & 5 & $205,457,1243,1405,1501$ \\
\hline BsaXI & ACNNNNNCTCC & 6 & three_prime & 6 & $348,378,1317,1347,1361,1391$ \\
\hline Hin4I & GAYNNNNNVTC & 6 & three_prime & 6 & $348,380,1060,1092,1317,1349$ \\
\hline HphI & GGTGA & 5 & three_prime & 6 & $211,908,971,1019,1495,1580$ \\
\hline TspGWI & ACGGA & 5 & three_prime & 6 & $178,530,979,1228,1291,1557$ \\
\hline $\mathrm{BccI}$ & CCATC & 5 & five_prime & 7 & $437,446,902,1031,1382,1423,1571$ \\
\hline AgsI & TTSAA & 5 & three_prime & 7 & $93,288,296,466,1045,1135,1290$ \\
\hline BbvI & GCAGC & 5 & five_prime & 8 & $138,339,357,543,874,1064,1103,1220$ \\
\hline TseI & GCWGC & 5 & five_prime & 8 & $151,327,370,531,887,1052,1091,1208$ \\
\hline TauI & GCSGC & 5 & three_prime & 8 & $31,546,951,1187,1256,1460,1463,1554$ \\
\hline
\end{tabular}

Table 32: Restriction digestion of protease gene from XM_025711220.1 Aspergillus vadensis

\begin{tabular}{|c|c|c|c|c|c|}
\hline Name & Sequence & Site length & Overhang & Frequency & Cut positions \\
\hline$\overline{\text { BalI }}$ & TGGCCA & 6 & blunt & 1 & 1054 \\
\hline BsaAI & YACGTR & 6 & blunt & 1 & 333 \\
\hline Eco47III & AGCGCT & 6 & blunt & 1 & 453 \\
\hline OliI & CACNNNNGTG & 6 & blunt & 1 & 416 \\
\hline PshAI & GACNNNNGTC & 6 & blunt & 1 & 682 \\
\hline PvuII & CAGCTG & 6 & blunt & 1 & 1129 \\
\hline SmaI & CCCGGG & 6 & blunt & 1 & 101 \\
\hline AvaI & CYCGRG & 6 & five_prime & 1 & 99 \\
\hline BamHI & GGATCC & 6 & five_prime & 1 & 958 \\
\hline BseYI & CCCAGC & 6 & five_prime & 1 & 265 \\
\hline BsmAI & GTCTC & 5 & five_prime & 1 & 1134 \\
\hline DraII & RGGNCCY & 6 & five_prime & 1 & 72 \\
\hline EcoRII & CCWGG & 5 & five_prime & 1 & 1103 \\
\hline MfeI & CAATTG & 6 & five_prime & 1 & 702 \\
\hline NcoI & CCATGG & 6 & five_prime & 1 & 1049 \\
\hline NheI & GCTAGC & 6 & five_prime & 1 & 526 \\
\hline PpuMI & RGGWCCY & 7 & five_prime & 1 & 72 \\
\hline SmlI & CTYRAG & 6 & five_prime & 1 & 1135 \\
\hline SpeI & ACTAGT & 6 & five_prime & 1 & 877 \\
\hline
\end{tabular}


Samia Abd Allah Abdal-Aziz and Safaa M. Ali / OnLine Journal of Biological Sciences 2021, 21 (1): 69.119 DOI: 10.3844/ojbsci.2021.69.119

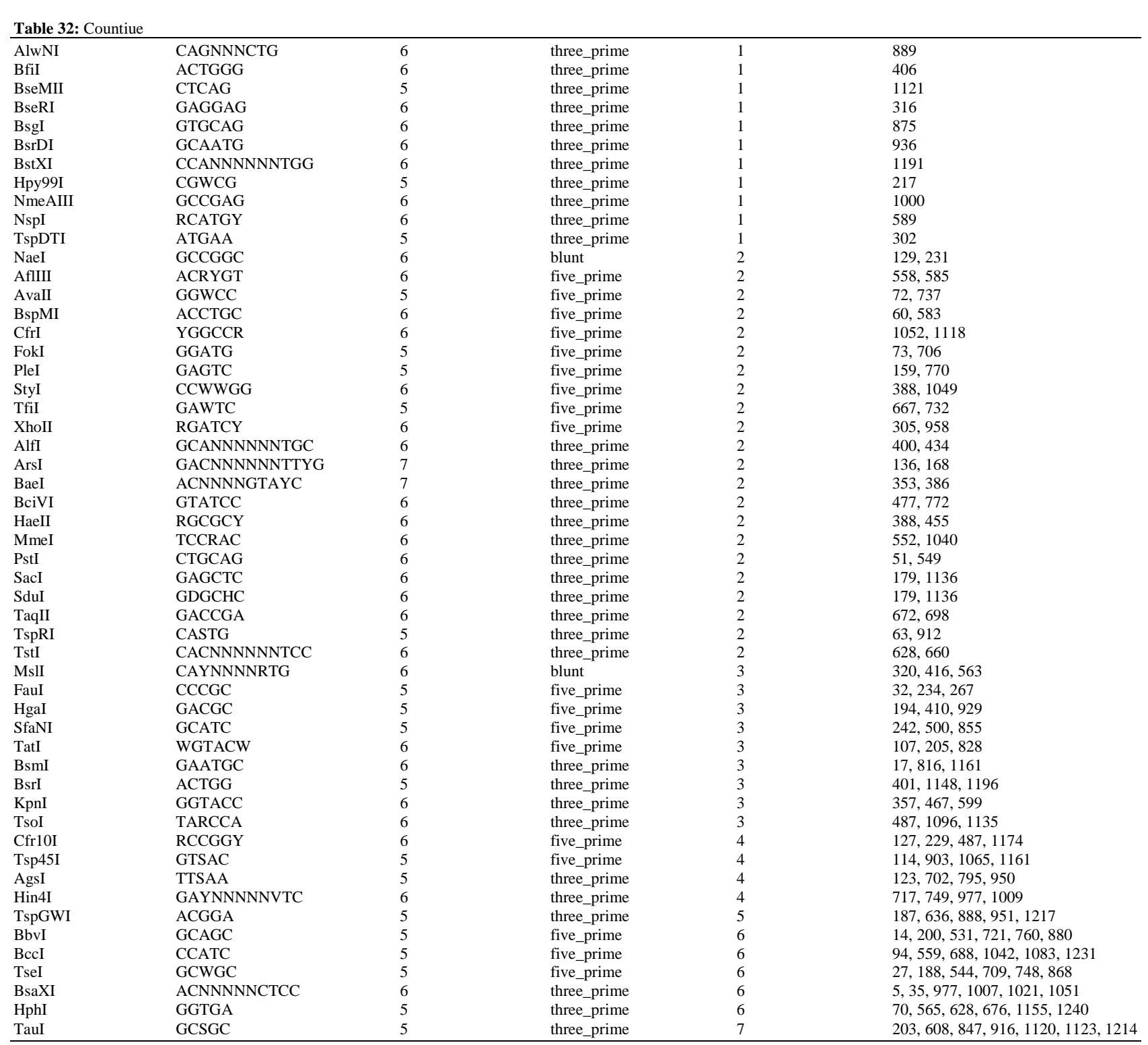

Table 33: Restriction digestion of protease gene from XM_024843524.1 Aspergillus steynii

\begin{tabular}{|c|c|c|c|c|c|}
\hline Name & Sequence & Site length & Overhang & Frequency & Cut positions \\
\hline$\overline{\text { BalI }}$ & TGGCCA & 6 & blunt & 1 & 1078 \\
\hline BsrBI & CCGCTC & 6 & blunt & 1 & 128 \\
\hline NaeI & GCCGGC & 6 & blunt & 1 & 459 \\
\hline $\mathrm{XmnI}$ & GAANNNNTTC & 6 & blunt & 1 & 247 \\
\hline AclI & AACGTT & 6 & five_prime & 1 & 500 \\
\hline AcyI & GRCGYC & 6 & five_prime & 1 & 962 \\
\hline AgeI & ACCGGT & 6 & five_prime & 1 & 382 \\
\hline AvaII & GGWCC & 5 & five_prime & 1 & 345 \\
\hline BspMI & ACCTGC & 6 & five_prime & 1 & 1094 \\
\hline BtgZI & GCGATG & 6 & five_prime & 1 & 1133 \\
\hline ClaI & ATCGAT & 6 & five_prime & 1 & 302 \\
\hline DraII & RGGNCCY & 6 & five_prime & 1 & 396 \\
\hline EcoP15I & CAGCAG & 6 & five_prime & 1 & 58 \\
\hline FokI & GGATG & 5 & five_prime & 1 & 163 \\
\hline HgaI & GACGC & 5 & five_prime & 1 & 561 \\
\hline NcoI & CCATGG & 6 & five_prime & 1 & 1049 \\
\hline NheI & GCTAGC & 6 & five_prime & 1 & 529 \\
\hline NotI & GCGGCCGC & 8 & five_prime & 1 & 522 \\
\hline SgrAI & CRCCGGYG & 8 & five_prime & 1 & 457 \\
\hline
\end{tabular}


Samia Abd Allah Abdal-Aziz and Safaa M. Ali / OnLine Journal of Biological Sciences 2021, 21 (1): 69.119 DOI: 10.3844/ojbsci.2021.69.119

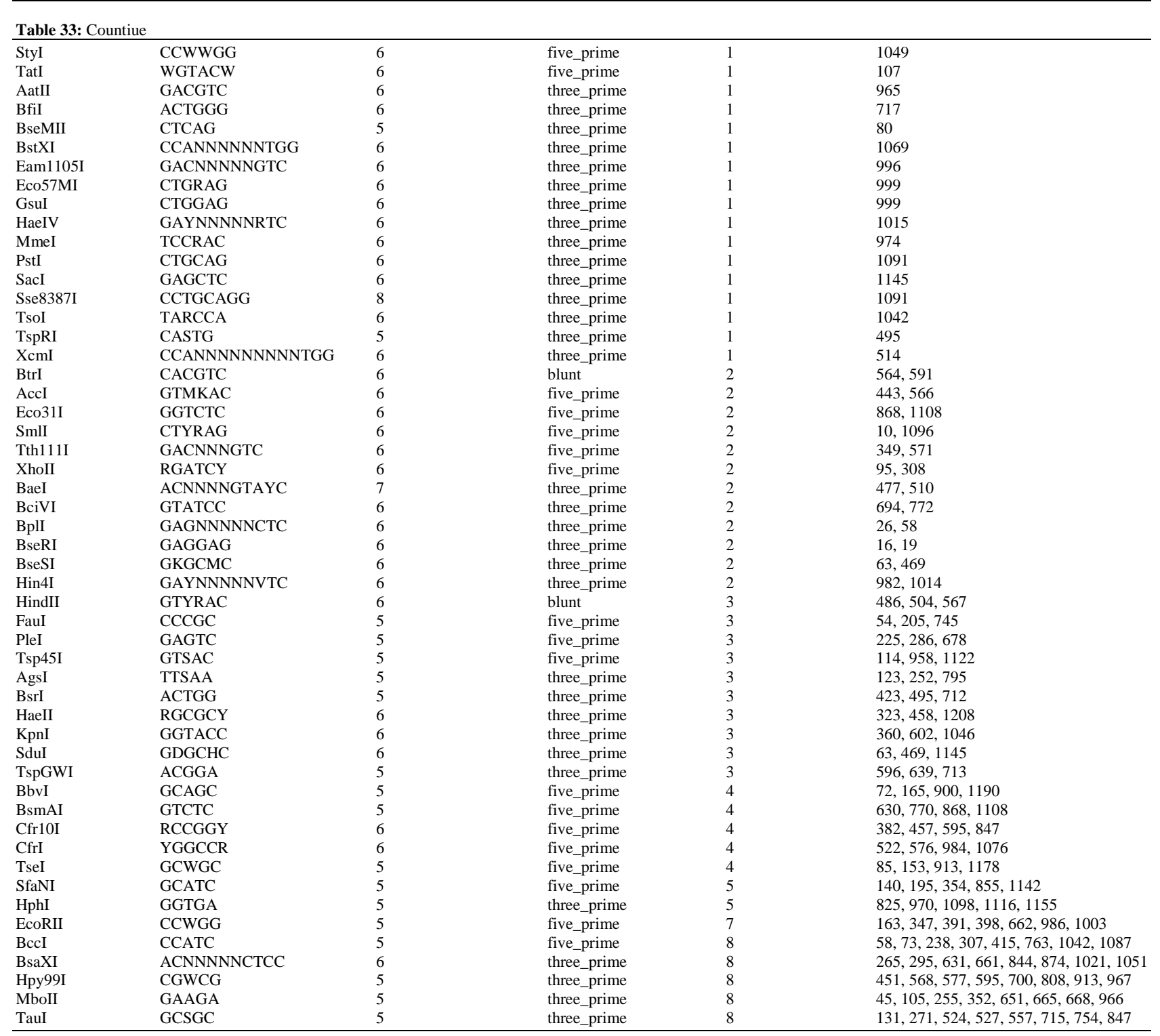

Table 34: Restriction digestion of protease gene from XM_033554602.1 Aspergillus lentulus

\begin{tabular}{|c|c|c|c|c|c|}
\hline Name & Sequence & Site length & Overhang & Frequency & Cut positions \\
\hline$\overline{\text { BsrBI }}$ & CCGCTC & 6 & blunt & 1 & 889 \\
\hline BtrI & CACGTC & 6 & blunt & 1 & 507 \\
\hline EcoRV & GATATC & 6 & blunt & 1 & 990 \\
\hline NruI & TCGCGA & 6 & blunt & 1 & 203 \\
\hline PvuII & CAGCTG & 6 & blunt & 1 & 1140 \\
\hline $\mathrm{XmnI}$ & GAANNNNTTC & 6 & blunt & 1 & 811 \\
\hline AcyI & GRCGYC & 6 & five_prime & 1 & 383 \\
\hline AscI & GGCGCGCC & 8 & five_prime & 1 & 59 \\
\hline AvaII & GGWCC & 5 & five_prime & 1 & 342 \\
\hline BsePI & GCGCGC & 6 & five_prime & 1 & 59 \\
\hline BtgZI & GCGATG & 6 & five_prime & 1 & 512 \\
\hline Eco31I & GGTCTC & 6 & five_prime & 1 & 1096 \\
\hline FauI & CCCGC & 5 & five_prime & 1 & 940 \\
\hline HindIII & AAGCTT & 6 & five_prime & 1 & 1186 \\
\hline NarI & GGCGCC & 6 & five_prime & 1 & 383 \\
\hline PfoI & TCCNGGA & 6 & five_prime & 1 & 68 \\
\hline SapI & GCTCTTC & 7 & five_prime & 1 & 235 \\
\hline SgrAI & CRCCGGYG & 8 & five_prime & 1 & 454 \\
\hline TfiI & GAWTC & 5 & five_prime & 1 & 96 \\
\hline Tth111I & GACNNNGTC & 6 & five_prime & 1 & 568 \\
\hline
\end{tabular}


Samia Abd Allah Abdal-Aziz and Safaa M. Ali / OnLine Journal of Biological Sciences 2021, 21 (1): 69.119 DOI: 10.3844/ojbsci.2021.69.119

\begin{tabular}{|c|c|c|c|c|c|}
\hline XhoI & CTCGAG & 6 & five_prime & 1 & 1093 \\
\hline BfiI & ACTGGG & 6 & three_prime & 1 & 714 \\
\hline BglI & GCCNNNNNGGC & 6 & three_prime & 1 & 1106 \\
\hline BseMII & CTCAG & 5 & three_prime & 1 & 103 \\
\hline BseRI & GAGGAG & 6 & three_prime & 1 & 352 \\
\hline BseSI & GKGCMC & 6 & three_prime & 1 & 133 \\
\hline BsrDI & GCAATG & 6 & three_prime & 1 & 1209 \\
\hline BtsI & GCAGTG & 6 & three_prime & 1 & 1016 \\
\hline Eco57MI & CTGRAG & 6 & three_prime & 1 & 214 \\
\hline GsuI & CTGGAG & 6 & three_prime & 1 & 214 \\
\hline HphI & GGTGA & 5 & three_prime & 1 & 1152 \\
\hline KpnI & GGTACC & 6 & three_prime & 1 & 599 \\
\hline SduI & GDGCHC & 6 & three_prime & 1 & 133 \\
\hline TsoI & TARCCA & 6 & three_prime & 1 & 764 \\
\hline TspDTI & ATGAA & 5 & three_prime & 1 & 833 \\
\hline NaeI & GCCGGC & 6 & blunt & 2 & 276,456 \\
\hline AccI & GTMKAC & 6 & five_prime & 2 & 482,965 \\
\hline Cfr10I & RCCGGY & 6 & five_prime & 2 & 274,454 \\
\hline EcoP15I & CAGCAG & 6 & five_prime & 2 & 814,1084 \\
\hline HgaI & GACGC & 5 & five_prime & 2 & 187,558 \\
\hline NcoI & CCATGG & 6 & five_prime & 2 & 388,1046 \\
\hline SalI & GTCGAC & 6 & five_prime & 2 & 481,964 \\
\hline AlfI & GCANNNNNNTGC & 6 & three_prime & 2 & 6,40 \\
\hline ArsI & GACNNNNNNTTYG & 7 & three_prime & 2 & 212,244 \\
\hline BplI & GAGNNNNNCTC & 6 & three_prime & 2 & 1087,1119 \\
\hline BsrI & ACTGG & 5 & three_prime & 2 & 492,709 \\
\hline Hin4I & GAYNNNNNVTC & 6 & three_prime & 2 & 717,749 \\
\hline MmeI & TCCRAC & 6 & three_prime & 2 & 971,1037 \\
\hline BalI & TGGCCA & 6 & blunt & 3 & $625,1051,1144$ \\
\hline BccI & CCATC & 5 & five_prime & 3 & $304,1039,1080$ \\
\hline PleI & GAGTC & 5 & five_prime & 3 & $159,675,770$ \\
\hline StyI & CCWWGG & 6 & five_prime & 3 & $30,388,1046$ \\
\hline Tsp45I & GTSAC & 5 & five_prime & 3 & $114,1119,1158$ \\
\hline AgsI & TTSAA & 5 & three_prime & 3 & $123,702,792$ \\
\hline HaeII & RGCGCY & 6 & three_prime & 3 & $386,455,461$ \\
\hline $\mathrm{BcgI}$ & CGANNNNNNTGC & 6 & three_prime & 4 & $93,127,505,539$ \\
\hline BsaXI & ACNNNNNCTCC & 6 & three_prime & 4 & $262,292,940,970$ \\
\hline FalI & AAGNNNNNCTT & 6 & three_prime & 4 & $812,844,867,899$ \\
\hline TspRI & CASTG & 5 & three_prime & 4 & $177,492,864,1023$ \\
\hline EcoRII & CCWGG & 5 & five_prime & 5 & $68,99,191,659,1000$ \\
\hline TauI & GCSGC & 5 & three_prime & 5 & $218,268,547,608,913$ \\
\hline TspGWI & ACGGA & 5 & three_prime & 5 & $593,632,636,948,1065$ \\
\hline Нpy99I & CGWCG & 5 & three_prime & 6 & $83,203,232,511,574,841$ \\
\hline MboII & GAAGA & 5 & three_prime & 6 & $105,252,642,648,662,963$ \\
\hline SfaNI & GCATC & 5 & five_prime & 7 & $126,276,351,500,535,852,1139$ \\
\hline BbvI & GCAGC & 5 & five_prime & 12 & $11,72,151,276,413,566,696,735,828,1101,1149,1187$ \\
\hline TseI & GCWGC & 5 & five_prime & 12 & $24,85,139,289,401,554,709,748,841,1114,1137,1175$ \\
\hline
\end{tabular}

Table 35: Restriction digestion of protease gene from XM_001258144.1 Aspergillus fischeri

\begin{tabular}{|c|c|c|c|c|c|}
\hline Name & Sequence & Site length & Overhang & Frequency & Cut positions \\
\hline$\overline{\mathrm{BsaAI}}$ & YACGTR & 6 & blunt & 1 & 15 \\
\hline EcoRV & GATATC & 6 & blunt & 1 & 1806 \\
\hline FspAI & RTGCGCAY & 8 & blunt & 1 & 1784 \\
\hline MslI & CAYNNNNRTG & 6 & blunt & 1 & 657 \\
\hline NaeI & GCCGGC & 6 & blunt & 1 & 336 \\
\hline ScaI & AGTACT & 6 & blunt & 1 & 424 \\
\hline StuI & AGGCCT & 6 & blunt & 1 & 139 \\
\hline AflIII & ACRYGT & 6 & five_prime & 1 & 12 \\
\hline ApoI & RAATTY & 6 & five_prime & 1 & 818 \\
\hline BglII & AGATCT & 6 & five_prime & 1 & 1052 \\
\hline BsePI & GCGCGC & 6 & five_prime & 1 & 1473 \\
\hline PasI & CCCWGGG & 7 & five_prime & 1 & 825 \\
\hline PfoI & TCCNGGA & 6 & five_prime & 1 & 109 \\
\hline SgrAI & CRCCGGYG & 8 & five_prime & 1 & 1692 \\
\hline SgrDI & CGTCGACG & 8 & five_prime & 1 & 853 \\
\hline TatI & WGTACW & 6 & five_prime & 1 & 422 \\
\hline AatII & GACGTC & 6 & three_prime & 1 & 1616 \\
\hline BciVI & GTATCC & 6 & three_prime & 1 & 935 \\
\hline BfiI & ACTGGG & 6 & three_prime & 1 & 751 \\
\hline BseSI & GKGCMC & 6 & three_prime & 1 & 832 \\
\hline BsgI & GTGCAG & 6 & three_prime & 1 & 410 \\
\hline DrallI & CACNNNGTG & 6 & three_prime & 1 & 685 \\
\hline
\end{tabular}


Samia Abd Allah Abdal-Aziz and Safaa M. Ali / OnLine Journal of Biological Sciences 2021, 21 (1): 69.119 DOI: 10.3844/ojbsci.2021.69.119

\begin{tabular}{|c|c|c|c|c|c|}
\hline Eco57I & CTGAAG & 6 & three_prime & 1 & 865 \\
\hline MmeI & TCCRAC & 6 & three_prime & 1 & 926 \\
\hline NspI & RCATGY & 6 & three_prime & 1 & 1659 \\
\hline PflMI & CCANNNNNTGG & 6 & three_prime & 1 & 935 \\
\hline SduI & GDGCHC & 6 & three_prime & 1 & 832 \\
\hline SphI & GCATGC & 6 & three_prime & 1 & 1659 \\
\hline Sse8387I & CCTGCAGG & 8 & three_prime & 1 & 1232 \\
\hline BsrBI & CCGCTC & 6 & blunt & 2 & 224,1731 \\
\hline PvuII & CAGCTG & 6 & blunt & 2 & 34,1852 \\
\hline SmaI & CCCGGG & 6 & blunt & 2 & 1527,1826 \\
\hline BamHI & GGATCC & 6 & five_prime & 2 & 1036,1866 \\
\hline BseYI & CCCAGC & 6 & five_prime & 2 & 642,1344 \\
\hline BspHI & TCATGA & 6 & five_prime & 2 & 192,1919 \\
\hline BtgZI & GCGATG & 6 & five_prime & 2 & 1167,1608 \\
\hline Cfr10I & RCCGGY & 6 & five_prime & 2 & 334,1692 \\
\hline CfrI & YGGCCR & 6 & five_prime & 2 & 937,1703 \\
\hline DraII & RGGNCCY & 6 & five_prime & 2 & 1140,1379 \\
\hline Eco31I & GGTCTC & 6 & five_prime & 2 & 169,283 \\
\hline Esp3I & CGTCTC & 6 & five_prime & 2 & 233,1242 \\
\hline NarI & GGCGCC & 6 & five_prime & 2 & 1163,1397 \\
\hline PpuMI & RGGWCCY & 7 & five_prime & 2 & 1140,1379 \\
\hline TfiI & GAWTC & 5 & five_prime & 2 & 5,898 \\
\hline Tth111I & GACNNNGTC & 6 & five_prime & 2 & 73,1633 \\
\hline AjuI & GAANNNNNNNTTGG & 7 & three_prime & 2 & 1003,1035 \\
\hline BaeI & ACNNNNGTAYC & 7 & three_prime & 2 & 1588,1621 \\
\hline $\mathrm{BcgI}$ & CGANNNNNNTGC & 6 & three_prime & 2 & 70,104 \\
\hline DrdI & GACNNNNNNGTC & 6 & three_prime & 2 & 1267,1420 \\
\hline GsuI & CTGGAG & 6 & three_prime & 2 & 56,1096 \\
\hline KpnI & GGTACC & 6 & three_prime & 2 & 1533,1679 \\
\hline PstI & CTGCAG & 6 & three_prime & 2 & 33,1232 \\
\hline TsoI & TARCCA & 6 & three_prime & 2 & 40,658 \\
\hline TstI & CACNNNNNNTCC & 6 & three_prime & 2 & 29,61 \\
\hline PleI & GAGTC & 5 & five_prime & 3 & $327,559,1255$ \\
\hline SmlI & CTYRAG & 6 & five_prime & 3 & $316,610,863$ \\
\hline StyI & CCWWGG & 6 & five_prime & 3 & $21,728,1136$ \\
\hline Tsp45I & GTSAC & 5 & five_prime & 3 & $455,1428,1872$ \\
\hline BseRI & GAGGAG & 6 & three_prime & 3 & $133,271,1741$ \\
\hline BsrDI & GCAATG & 6 & three_prime & 3 & $461,493,741$ \\
\hline Eco57MI & CTGRAG & 6 & three_prime & 3 & $56,865,1096$ \\
\hline HindII & GTYRAC & 6 & blunt & 4 & $855,1032,1551,1751$ \\
\hline AvalI & GGWCC & 5 & five_prime & 4 & $261,1065,1140,1379$ \\
\hline EcoRII & CCWGG & 5 & five_prime & 4 & $109,284,522,824$ \\
\hline SalI & GTCGAC & 6 & five_prime & 4 & $853,1030,1549,1749$ \\
\hline HaeII & RGCGCY & 6 & three_prime & 4 & $863,1166,1197,1400$ \\
\hline HaeIV & GAYNNNNNRTC & 6 & three_prime & 4 & $281,315,953,987$ \\
\hline MbolI & GAAGA & 5 & three_prime & 4 & $135,1320,1429,1635$ \\
\hline NmeAIII & GCCGAG & 6 & three_prime & 4 & $1191,1202,1233,1373$ \\
\hline TspRI & CASTG & 5 & three_prime & 4 & $686,1005,1120,1207$ \\
\hline AccI & GTMKAC & 6 & five_prime & 5 & $854,1031,1124,1550,1750$ \\
\hline AvaI & CYCGRG & 6 & five prime & 5 & $1309,1443,1525,1789,1824$ \\
\hline BsmAI & GTCTC & 5 & five_prime & 5 & $120,169,233,283,1242$ \\
\hline EcoP15I & CAGCAG & 6 & five_prime & 5 & $612,1516,1587,1654,1657$ \\
\hline XholI & RGATCY & 6 & five_prime & 5 & $756,783,1036,1052,1866$ \\
\hline BsrI & ACTGG & 5 & three_prime & 5 & $134,746,1005,1113,1200$ \\
\hline HphI & GGTGA & 5 & three_prime & 5 & $510,1297,1356,1422,1884$ \\
\hline TspDTI & ATGAA & 5 & three_prime & 5 & $98,572,752,1231,1908$ \\
\hline TspGWI & ACGGA & 5 & three_prime & 5 & $130,240,1049,1230,1330$ \\
\hline AgsI & TTSAA & 5 & three_prime & 6 & $403,574,803,916,1011,1647$ \\
\hline BsaXI & ACNNNNNCTCC & 6 & three_prime & 6 & $29,59,1039,1069,1495,1525$ \\
\hline BseMII & CTCAG & 5 & three_prime & 6 & $184,314,364,410,1292,1862$ \\
\hline AcyI & GRCGYC & 6 & five_prime & 7 & $1121,1163,1235,1268,1397,1547,1613$ \\
\hline HgaI & GACGC & 5 & five_prime & 7 & $391,1110,1224,1257,1536,1607,1671$ \\
\hline SfaNI & GCATC & 5 & five_prime & 7 & $353,536,755,1014,1261,1589,1794$ \\
\hline FauI & CCCGC & 5 & five_prime & 8 & $229,602,1076,1092,1369,1414,1444,1522$ \\
\hline BccI & CCATC & 5 & five_prime & 9 & $198,847,937,958,1156,1189,1630,1714,1856$ \\
\hline BbvI & GCAGC & 5 & five_prime & 10 & $15,43,403,626,1500,1571,1630,1671,1674,1839$ \\
\hline FokI & GGATG & 5 & five_prime & 10 & $778,821,826,937,1036,1227,1239,1693,1772,1874$ \\
\hline TseI & GCWGC & 5 & five_prime & 10 & $28,31,391,639,1488,1559,1618,1684,1687,1852$ \\
\hline TauI & GCSGC & 5 & three_prime & 11 & $618,716,942,1097,1121,1163,1199,1451,1580,1705,1734$ \\
\hline Hin4I & GAYNNNNNVTC & 6 & three_prime & 16 & $124,147,156,179,208,230,240,262,282,314,331,363,954,986,1495,1527$ \\
\hline Hрy99I & CGWCG & 5 & three_prime & 16 & $92,256,298,856,859,1036,1093,1246,1249,1252,1273,1501,1552,1618,1639,1665$ \\
\hline
\end{tabular}

Table 36: Restriction digestion of protease gene from XM_033555156.1 Aspergillus lentulus

\begin{tabular}{|c|c|c|c|c|c|}
\hline Name & Sequence & Site length & Overhang & Frequency & Cut positions \\
\hline Eco47III & AGCGCT & 6 & blunt & 1 & 734 \\
\hline HindII & GTYRAC & 6 & blunt & 1 & 1057 \\
\hline MslI & CAYNNNNRTG & 6 & blunt & 1 & 287 \\
\hline NaeI & GCCGGC & 6 & blunt & 1 & 387 \\
\hline PvuII & CAGCTG & 6 & blunt & 1 & 790 \\
\hline AccI & GTMKAC & 6 & five_prime & 1 & 328 \\
\hline AclI & AACGTT & 6 & five_prime & 1 & 578 \\
\hline AcyI & GRCGYC & 6 & five_prime & 1 & 587 \\
\hline
\end{tabular}




\begin{tabular}{|c|c|c|c|c|c|}
\hline AflIII & ACRYGT & 6 & five_prime & 1 & 435 \\
\hline BglII & AGATCT & 6 & five_prime & 1 & 14 \\
\hline Cfr10I & RCCGGY & 6 & five_prime & 1 & 385 \\
\hline HgaI & GACGC & 5 & five_prime & 1 & 576 \\
\hline NdeI & CATATG & 6 & five_prime & 1 & 405 \\
\hline PpuMI & RGGWCCY & 7 & five_prime & 1 & 718 \\
\hline SmlI & CTYRAG & 6 & five_prime & 1 & 646 \\
\hline AlwNI & CAGNNNCTG & 6 & three_prime & 1 & 616 \\
\hline ApaI & GGGCCC & 6 & three_prime & 1 & 469 \\
\hline BseRI & GAGGAG & 6 & three_prime & 1 & 1018 \\
\hline BseSI & GKGCMC & 6 & three_prime & 1 & 469 \\
\hline BsmI & GAATGC & 6 & three_prime & 1 & 1146 \\
\hline BsrDI & GCAATG & 6 & three_prime & 1 & 294 \\
\hline BtsI & GCAGTG & 6 & three_prime & 1 & 282 \\
\hline EciI & GGCGGA & 6 & three_prime & 1 & 133 \\
\hline Eco57I & CTGAAG & 6 & three_prime & 1 & 148 \\
\hline HaeII & RGCGCY & 6 & three_prime & 1 & 736 \\
\hline KpnI & GGTACC & 6 & three_prime & 1 & 524 \\
\hline NmeAIII & GCCGAG & 6 & three_prime & 1 & 491 \\
\hline NspI & RCATGY & 6 & three_prime & 1 & 439 \\
\hline SduI & GDGCHC & 6 & three_prime & 1 & 469 \\
\hline BalI & TGGCCA & 6 & blunt & 2 & 4,1063 \\
\hline ApoI & RAATTY & 6 & five_prime & 2 & 205,442 \\
\hline AvalI & GGWCC & 5 & five_prime & 2 & 349,718 \\
\hline Bpu10I & CCTNAGC & 6 & five_prime & 2 & 83,266 \\
\hline BseYI & CCCAGC & 6 & five_prime & 2 & 50,790 \\
\hline BsmAI & GTCTC & 5 & five_prime & 2 & 672,1030 \\
\hline FauI & CCCGC & 5 & five_prime & 2 & 525,831 \\
\hline NcoI & CCATGG & 6 & five_prime & 2 & 516,989 \\
\hline TatI & WGTACW & 6 & five_prime & 2 & 90,507 \\
\hline TfiI & GAWTC & 5 & five_prime & 2 & 184,1112 \\
\hline ArsI & GACNNNNNNTTYG & 7 & three_prime & 2 & 463,495 \\
\hline $\mathrm{BcgI}$ & CGANNNNNNTGC & 6 & three_prime & 2 & 820,854 \\
\hline BciVI & GTATCC & 6 & three_prime & 2 & 187,233 \\
\hline BsaXI & ACNNNNNCTCC & 6 & three_prime & 2 & 91,121 \\
\hline $\mathrm{XcmI}$ & CCANNNNNNNNNTGG & 6 & three_prime & 2 & 228,305 \\
\hline EcoRV & GATATC & 6 & blunt & 3 & $489,633,864$ \\
\hline DraII & RGGNCCY & 6 & five_prime & 3 & $465,642,718$ \\
\hline EcoP15I & CAGCAG & 6 & five_prime & 3 & $196,715,992$ \\
\hline EcoRII & CCWGG & 5 & five_prime & 3 & $176,880,921$ \\
\hline FokI & GGATG & 5 & five_prime & 3 & $193,517,972$ \\
\hline PleI & GAGTC & 5 & five_prime & 3 & $135,716,825$ \\
\hline StyI & CCWWGG & 6 & five_prime & 3 & $516,989,1043$ \\
\hline HphI & GGTGA & 5 & three_prime & 3 & $296,613,711$ \\
\hline TsoI & TARCCA & 6 & three_prime & 3 & $558,982,1036$ \\
\hline TspGWI & ACGGA & 5 & three_prime & 3 & $142,812,874$ \\
\hline BbvI & GCAGC & 5 & five_prime & 4 & $282,401,729,799$ \\
\hline CfrI & YGGCCR & 6 & five_prime & 4 & $2,513,687,1061$ \\
\hline SfaNI & GCATC & 5 & five_prime & 4 & $111,729,1116,1164$ \\
\hline TseI & GCWGC & 5 & five_prime & 4 & $270,389,742,787$ \\
\hline AgsI & TTSAA & 5 & three_prime & 4 & $188,262,442,755$ \\
\hline MboII & GAAGA & 5 & three_prime & 4 & $142,154,238,579$ \\
\hline TauI & GCSGC & 5 & three_prime & 4 & $323,395,689,956$ \\
\hline TspRI & CASTG & 5 & three_prime & 4 & $289,402,1038,1151$ \\
\hline BseMII & CTCAG & 5 & three_prime & 5 & $74,257,357,902,1044$ \\
\hline BsrI & ACTGG & 5 & three_prime & 5 & $423,545,1064,1151,1163$ \\
\hline Hin4I & GAYNNNNNVTC & 6 & three_prime & 6 & $91,123,365,397,922,954$ \\
\hline
\end{tabular}

\section{Amino Acid Sequences of Asperigillus Protease >A CAA75805.1Aspergillus fumigatus}

Krnlerrdttsgeppvgieksykikdfaayagsfddatieeirksadvah veedqiwyldalttqkgapwglgsishkgqastdyiydtsagagtyayvvd sginvnhvefesraslaynaaggshvdsighgthvagtiggktygvakktnll svkvfqgessstsiildgfnwavndivskgrtkkaainmslgggysyafnna venafdegvlsvvaagnensdasntspasapnaltvaainksnarasfsnyg svvdifapgqdilsawigsttatntisgtsmatphivglsvylmglenlsgpaa vtarikelatngvvtnvkgspnklayngn

\section{>XP_751651.1 Aspergillus fumigatus}

Mlsikrtllllgavlpavfgapvqetrraaqkipgkyivtfkpgtdtaties htlwatdlhkrnlerrdttsgeppvgieksykikdfaayagsfddatieeirks advahveedqiwyldalttqkgapwglgsishkgqastdyiydtsagagty ayvvdsginvnhvefesraslaynaaggshvdsighgthvagtiggktygv akktnllsvkvfqgessstsiildgfnwavndivskgrtkkaainmslgggys 
yafnnavenafdegvlsvvaagnensdasntspasapnaltvaainksnara sfsnygsvvdifapgqdilsawigsttatntisgtsmatphivglsvylmglen lsgpaavtarikelatngvvtnvkgspnklayngna

\section{>CAA77666.1 Aspergillus fumigatus}

Mlsikrtllllgavlpavfgapvqetrraaqkipgkyivtfkpgtdtaties htlwatdlhkrnlerrdttsgeppvgieksykikdfaayagsfddatieeirkr gdvahveedqiwyldalttqkgapwglgsishkgqastdyiydtsagagty ayvvdsginvnhvefesraslaynaaggshvdsighgthvagtiggktygv akktnllsvkvfqgessstsiildgfnwavndivskgrtkkaainmslgggys yafnnavenafdegvlsvvaagnensdasntspasapnaltvaainksnara sfsnygsvvdifapgqdilsawigsttatntisgtsmatphivglsvylmglen lsgpaavtarikelatngvvtnvkgspnklayngna

\section{>XP_750914.1 Aspergillus fumigatus}

Mrlshvllgtaaaagvlasptpndyvvherravlprswteekrldka silpmrigltqsnldrghdllmeisdprssrygqhlsveevhslfapsqetv drvrawlesegiagdrisqssneqflqfdasaaeverllgteyylythqgs gkshiacreyhvphslqrhidyitpgikllevegvkkarsiekrsfrsplpp ilerltlplsellgntllcdvaitplcisalynitrgskatkgnelgifedlgdvy sqedlnlffstfaqqipqgthpilkavdgaqaptsvtnagpesdldfqisy piiwpqnsilfqtddpnytanynfsgflntfldaidgsycseispldppyp npadggykgqlqcgvyqppkvlsisyggaeadlpiayqrrqcaewmk lglqgvsvvvasgdsgvegrngdptpteclgtegkvfapdfpatcpyltt vggtylplgadprkdeevavtsfpsgggfsniyeradyqqqavedyfsra dpgypfyesvdnssfaenggiynrigraypdvaaiadnvvifnkgmptl iggtsaaapvfaailtrineerlavgkstvgfvnpvlyahpevfnditqgsn pgcgmqgfsaatgwdpvtglgtpnypalldlfmslp

\section{>EDP50543.1 Aspergillus fumigatus}

Mlsikrtllllgavlpavfgapvqetrraaqkipgkyivtfkpgtdtaties htlwatdlhkrnlerrdttsgeppvgieksykikdfaayagsfddatieeirks advahveedqiwyldalttqkgapwglgsishkgqastdyiydtsagagty ayvvdsginvnhvefesraslaynaaggshvdsighgthvagtiggktygv akktnllsvkvfqgessstsiildgfnwavndivskgrtkkaainmslgggys yafnnavenafdegvlsvvaagnensdasntspasapnaltvaainksnara sfsnygsvvdifapgqdilsawigsttatntisgtsmatphivglsvylmglen lsgpaavtarikelatngvvtnvkgspnklayngna

\section{>1905286A1 Aspergillus fumigatus}

Mlsikrtslllgavlpavfgapvqetrraaqkipgkyivtfkpgtdtaties htlwatdlhkrnlerrdttsgeppvgieksykikdfaayagsfddatieeirkr gdvahveedqiwyldalttqkgapwglgsishkgqastdyiydtsagagty ayvvdsginvnhvefesraslaynaaggshvdsighgthvagtiggkttgva kktnllsvkvfqgessstsiildgfnwavndivskgrtkkaainmslgggysy afnnavenafdegvlsvvaagnensdasntspasapnaltvaainksnaras fsnygsvvdifapgqdilsawigsttatntisgtsmatphivglsvylmglenl sgpaavtarikelatngvvtnvkgspnklayngna

\section{>XP_751651.1 Aspergillus fumigatus}

Mlsikrtllllgavlpavfgapvqetrraaqkipgkyivtfkpgtdtaties htlwatdlhkrnlerrdttsgeppvgieksykikdfaayagsfddatieeirks advahveedqiwylwglgsishkgqastdyiydtsagagtyayvvdsginv nhvefesraslaynaaggshvdsighgthvagtiggktygvakktnllsvkv fqgessstsiildgfnwavndivskgrtkkaainmslgggysyafnnavena fdegvlsvvaagnensdasntspasapnaltvaainksnarasfsnygsvvd ifapgqdilsawigsttatntisgtsmatphivglsvylmglenlsgpaavtari kelatngvvtnvkgspnklayngna

\section{>B0Y708.1 Aspergillus fumigatus}

Mlsikrtllllgavlpavfgapvqetrraaqkipgkyivtfkpgtdtaties htlwatdlhkrnlerrdttsgeppvgieksykikdfaayagsfddatieeirks advahveedqiwyldalttqkgapwglgsishkgqastdyiydtsagagty ayvvdsginvnhvefesraslaynaaggshvdsighgthvagtiggktygv akktnllsvkvfqgessstsiildgfnwavndivskgrtkkaainmslgggys yafnnavenafdegvlsvvaagnensdasntspasapnaltvaainksnara sfsnygsvvdifapgqdilsawigsttatntisgtsmatphivglsvylmglen lsgpaavtarikelatngvvtnvkgspnklayngna

\section{>Q4WH48 Aspergillus fumigatus}

Mfffvpaftsdqtdairghaqvadayipkgqlmvrfwgvarnptsesg ppagdddwfmdtlnsteselqersilakrseivernspdnmvslswppdig pvpvqgdyrfdssagegtyvyhvdfgaqpshpefsdvsflhpllpgpypvs gwmendpkrhgslclskevgktvgiarkatvvatawdfqksinehwldal akvhadistgargaksvvnlsisipqgdltaaflekmallireiiklgavfvtgs gnspgspngypalfgdpanpnhipelivvgsvlgqgilgqhanadwvtcy apgyglrmadsdpesateyrttqgtsfasatvaglaayfrgldstlttaasvker ilrlayrrqpqpnhpegpyqryidnvvwngqkwgrsivpecsdfskakrqs sggscpvafppqpspltfrtgppqptcagagcgsscagffcpgtplkqnpdfl dprnpdsvqnpdsryyedwdgtitrttttptktiptttptppksssvpiggpcrl tdecedncpkpgavqcesgactcltpppkttpphaamcydvqqclnvytc asgdtmvceptdysngnglcqcikgnps

\section{>CAA75806.1 Aspergillus fumigatus}

Krnlerrdstsgdtpvgieknykindfaayagsfddatieeirkssdvah veedqiwhinalttqkgapwglgsishkgqastdyiydttagagtyayvvdt ginvnhvefggraslaynaaggthvdsvghgthvagtiggstygvakktnll svkvfqgessstsiildgfnwaandivskgrtkkaainmslgggysyafnna vetaftsgvlsvvaagnentdasdtspasapnaltvaaisksnarasfsnygsv vdifapgqsilsawigsntatntisgtsmatphivglsvylmglenlsgpaavt arikqlatsgvvtdaqgspnklayngn

\section{>CAA75804.1 Aspergillus fumigatus}

Krnlerrdassgdtpvgieknykikdfaayagsfddatieeirksadvah veedqiwyidalttqrgapwglgsishkgqastdyiydtsagagtyayvvdt ginvnhvefegraslaynaaggshvdsvghgthvagtiggktygvakktnll svkvfqgessstsiildgfnwaandivskgrtrkaainmslgggysyafnna venafdegvlsvvaagnentdasetspasapnaltvaainrsnarasfsnygs vvdifapgqdilsawigsntatntisgtsmatphivglsvylmglenlsgpaa vtsrikqlatngvvtdtrgspnklayngn 


\section{>EAL89613.1 Aspergillus fumigatus}

mlsikrtllllgavlpavfgapvqetrraaqkipgkyivtfkpgtdtaties htlwatdlhkrnlerrdttsgeppvgieksykikdfaayagsfddatieeirks advahveedqiwyldalttqkgapwglgsishkgqastdyiydtsagagty ayvvdsginvnhvefesraslaynaaggshvdsighgthvagtiggktygv akktnllsvkvfqgessstsiildgfnwavndivskgrtkkaainmslgggys yafnnavenafdegvlsvvaagnensdasntspasapnaltvaainksnara sfsnygsvvdifapgqdilsawigsttatntisgtsmatphivglsvylmglen lsgpaavtarikelatngvvtnvkgspnklayngna $>$ XP_748781.1As pergillusfumigatusmfffvpaftsdqtdairghaqvadayipkgqlmv rfwgvarnptsesgppagdddwfmdtlnsteselqersilakrseivernsp dnmvslswppdigpvpvqgdyrfdssagegtyvyhvdfgaqpshpefsd vsflhpllpgpypvsgwmendpkrhgslclskevgktvgiarkatvvataw dfqksinehwldalakvhadistgargaksvvnlsisipqgdltaaflekmal lireiiklgavfvtgsgnspgspngypalfgdpanpnhipelivvgsvlgqgil gqhanadwvtcyapgyglrmadsdpesateyrttqgtsfasatvaglaayfr gldstlttaasvkerilrlayrrqpqpnhpegpyqryidnvvwngqkwgrsi vpecsdfskakrqssggscpvafppqpspltfrtgppqptcagagcgsscag ffcpgtplkqnpdfldprnpdsvqnpdsryyedwdgtitrtttptktiptttpt ppksssvpiggpcrltdecedncpkpgavqcesgactcltpppkttpphaa mcydvqqclnvytcasgdtmvceptdysngnglcqcikgnps

\section{>EAL86979.1 Aspergillus fumigatus}

Matqhfmttgqttvpgqwvvrvkpyltpelvqkehlslleektedpat pfnveilqrfdlydskgysakfddatkeelekiphvvsiepeqlyrhcniqpn spwgisrvstrtklgappysytyrddvagsgtvayvidtginnkhvefegra qkgpkfvsdnvsndedvhghgthcagtiasraygvakkanvvgvkvfgdr tgtaqtsdiikalewvisdisakgmggravvnlslggppsdaldaavastvh kgvvvcdiqespareplaitvgatdikdqlanfssygkfvdilapgvdilscw tggptstktisgtsmatphvggvaccllsdptlaggqattydvmskililadkn kitgtdartvnallhnttspmda

\section{>XP_749017.1 Aspergillus fumigatus}

Matqhfmttgqttvpgqwvvrvkpyltpelvqkehlslleektedpat pfnveilqrfdlydskgysakfddatkeelekiphvvsiepeqlyrhcniqpn spwgisrvstrtklgappysytyrddvagsgtvayvidtginnkhvefegra qkgpkfvsdnvsndedvhghgthcagtiasraygvakkanvvgvkvfgdr tgtaqtsdiikalewvisdisakgmggravvnlslggppsdaldaavastvh kgvvvcdiqespareplaitvgatdikdqlanfssygkfvdilapgvdilscw tggptstktisgtsmatphvggvaccllsdptlaggqattydvmskililadkn kitgtdartvnallhnttspmda

\section{>B0Y1V8.1 Aspergillus fumigatus}

Mvvfskvtavvvglstivsavpvvqprkgftinqvarpvtnkktvnlp avyanaltkyggtvpdsvkaaassgsavttpeqydseyltpvkvggttlnldf dtgsadlwvfsselsasqssghaiykpsanaqklngytwkiqygdgssasg dvykdtvtvggvtaqsqaveaashissqfvqdkdndgllglafssintvsprp qttffdtvksqldsplfavtlkyhapgtydfgyidnskfqgeltytdvdssqgf wmftadgygvgngapnsnsisgiadtgttllllddsvvadyyrqvsgakns nqyggyvfpcstklpsfttviggynavvpgeyinyapvtdgsstcyggiqsn sglgfsifgdiflksqyvvfdsqgprlgfapqa

\section{>AAT85627.1 Aspergillus viridinutans}

Krnlerrdttssgdapvgieknykikdfaayagsfdaatieeirksadva hveedqvwyinslttqkgapwglgsishkgqastdyiydtsagagtyayvv dtginvnhvefggraslaynaaggnhvdsvghgthvagtiggstygvakkt nllsvkvfqgessstsiildgfnwaandivskgrtkkaainmslgggysyafn navenafqegvlsvvaagnensdasdtspasapnavtvaaisknnarasfsn ygsvvdifapgqnilsawigsntatntisgtsmatphivglsv

\section{>AAT85626.1 Aspergillus viridinutans}

Krnlerrdttssgdapvgieknykikdfaayagsfdaatieeirksadva hveedqvwyinslttqkgapwglgsishkgqastdyiydtsagagtyayvv dtginvnhvefggraslaynaaggnhvdsvghgthvagtiggstygvakkt nllsvkvfqgessstsiildgfnwavndivskgrtskaainmslgggysyafn navenafqegvlsvvaagnensdasntspasapnavtvaaisknnarasfsn ygsvvdifapgqnilsawigsntatntisgtsmatphivglsvylmglenlsg paavtarikqlatngvvtdaqg

\section{>A1CIA7.1 Aspergillus clavatus}

Mqsikrtllllgallpaalaaparephpssniipgkyiitfksgidtaaiesh tawasnihkrnlerrglvggefpagierkfkikdfaayagsfdpatieeirnse dvahveedqiwyldalttqsgapwglgsishkgqastnyvydtsagagtya yvvdsginvdhiefqgratkaynavggdhvdtlghgthvagtiggktygva kqtnllsvkvfegrtgstsvildgfnwaandivskgrkgkaainmslgggys yafnnavesayeqgvlsvvaagnegvdasnsspasapnaltvgatnksnar asfsnygkvldifapgqdilsawigsttatntisgtsmatphvvglavylmgl egvsgpaavtqrilqlatsgvisdvkgspnklayngaa

\section{>A1CBR4.1 Aspergillus clavatus}

Mvvfskvtaavfglatiasaapapptrkgftvqqqarpaqkkqvnlpa myahaltkfggsvpesvkvaaskgsavttpeagdveyltpvnvggtvmnl dfdtgsadlwvfsgelpasetsghsvykpgrtasklpggswqisygdgssas gdvykdtvvvggvtahgqaveaaaqissqflqdknndgllglafsslntvqp qpqttffdtvkssldrplfavtlkhnapgsfdfgyidhskytgeiaytdvdnsq gfwsftadgysigggqssgssisgiadtgttllllddnvvsdfyqhvegaqns deyggyvfpcsakvpsfttiiggykavtpgklinygpvtdgsstcyggiqss ggvgqnifgdiflksqfvvfdsegprlgfaaqa

\section{>A1CBR4.1 Aspergillus clavatus}

Mvvfskvtaavfglatiasaapapptrkgftvqqqarpaqkkqvnlpa myahaltkfggsvpesvkvaaskgsavttpeagdveyltpvnvggtvmnl dfdtgsadlwvfsgelpasetsghsvykpgrtasklpggswqisygdgssas gdvykdtvvvggvtahgqaveaaaqissqflqdknndgllglafsslntvqp qpqttffdtvkssldrplfavtlkhnapgsfdfgyidhskytgeiaytdvdnsq gfwsftadgysigggqssgssisgiadtgttllllddnvvsdfyqhvegaqns deyggyvfpcsakvpsfttiiggykavtpgklinygpvtdgsstcyggiqss ggvgqnifgdiflksqfvvfdsegprlgfaaqa

\section{>ACX47962.1 Aspergillus clavatus}

Mqsikrtllllgallpaalaapvrephpssniipgkyiitfksgtdtaaiesh tawasnihkrnlerrglvggefpagiekkfkikdfaayagsfdpttieeirnsa 
dvahveedqiwyldalttqsgapwglgsishkgqastnyvydtsagagtya yvvdsginvnhiefqgratkaynavggdhvdtlghgthvagtiggktygva kqtnllsvkvfegrtsstsvildgfnwaandivskgrkgkaainmslgggys yafnnavesayeqgvlsvvaagnegvdasnsspasapnaltvgatnksnar asfsnygkvldifapgqdilsawigsstatntisgtsmatphvvglavylmgl egvsgpaavtqrilqlatsgvisdvkgspnklayngaa

\section{>EAW10612. 1 Aspergillus clavatus}

Mqsikrtllllgallpaalaaparephpssniipgkyiitfksgidtaaiesh tawasnihkrnlerrglvggefpagierkfkikdfaayagsfdpatieeirnse dvahveedqiwyldalttqsgapwglgsishkgqastnyvydtsagagtya yvvdsginvdhiefqgratkaynavggdhvdtlghgthvagtiggktygva kqtnllsvkvfegrtgstsvildgfnwaandivskgrkgkaainmslgggys yafnnavesayeqgvlsvvaagnegvdasnsspasapnaltvgatnksnar asfsnygkvldifapgqdilsawigsttatntisgtsmatphvvglavylmgl egvsgpaavtqrilqlatsgvisdvkgspnklayngaa

\section{>EAW10612.1 Aspergillus clavatus}

Mqsikrtllllgallpaalaaparephpssniipgkyiitfksgidtaaiesh tawasnihkrnlerrglvggefpagierkfkikdfaayagsfdpatieeirnse dvahveedqiwyldalttqsgapwglgsishkgqastnyvydtsagagtya yvvdsginvdhiefqgratkaynavggdhvdtlghgthvagtiggktygva kqtnllsvkvfegrtgstsvildgfnwaandivskgrkgkaainmslgggys yafnnavesayeqgvlsvvaagnegvdasnsspasapnaltvgatnksnar asfsnygkvldifapgqdilsawigsttatntisgtsmatphvvglavylmgl egvsgpaavtqrilqlatsgvisdvkgspnklayngaa

\section{>XP_001272038 Aspergillus clavatus}

Mqsikrtllllgallpaalaaparephpssniipgkyiitfksgidtaaiesh tawasnihkrnlerrglvggefpagierkfkikdfaayagsfdpatieeirnse dvahveedqiwyldalttqsgapwglgsishkgqastnyvydtsagagtya yvvdsginvdhiefqgratkaynavggdhvdtlghgthvagtiggktygva kqtnllsvkvfegrtgstsvildgfnwaandivskgrkgkaainmslgggys yafnnavesayeqgvlsvvaagnegvdasnsspasapnaltvgatnksnar asfsnygkvldifapgqdilsawigsttatntisgtsmatphvvglavylmgl egvsgpaavtqrilqlatsgvisdvkgspnklayngaa

\section{>AAA67705.1 Aspergillus nidulans}

Mhsfkrsllllgallpavfgapveprraaekvpgkyivtfksglnvdqid ahtswasnvhkrnlerrglaerdqysgieknykinkfaaysgsfddatieeir nsadvahveedqiwyidaltsqsgapwglgaishkgeasttyvydtsagegt yayvvdtginadheefggraslaynavggqhvdsvghgthvagtiggetyg vskkanllsvkvfqgessstsiildgfnwaandivskgrtgksainmslggg ysyafnqavedaydegvlsvvaagndnidasdsspasapnaltvaastksnt rasfsnygsvvdifapgqdilsawigsttatntisgtsmatphvvglslyliale glssasavvsrikelatqgvlsnvqgspnllayngade

\section{>Q00208.1 Aspergillus nidulans}

Mhsfkrsllllgallpavfgapveprraaekvpgkyivtfksglnvdqid ahtswasnvhkrnlerrglaerdqysgieknykinkfaaysgsfddatieeir nsadvahveedqiwyidaltsqsgapwglgaishkgeasttyvydtsagegt yayvvdtginadheefggraslaynavggqhvdsvghgthvagtiggetyg vskkanllsvkvfqgessstsiildgfnwaandivskgrtgksainmslggg ysyafnqavedaydegvlsvvaagndnidasdsspasapnaltvaastksnt rasfsnygsvvdifapgqdilsawigsttatntisgtsmatphvvglslyliale glssasavvsrikelatqgvlsnvqgspnllayngade

\section{>Q00204.2 Aspergillus nidulans}

Msrtssapsqkslisralkaerdvitassqsqaldaaidaaehymkalalt ssskdrnvldakckewltraekikgsedwrsvaqsrrsrlrtpastrklttredii llqgaklngfifppwkaepsltefetgtngdvlftdkpdlhlsnlqrdifagwk rphellsgqvddagmplnpvmtvsgntdlvqdvltdcsvvaslcattsrser glddtllpivypcihnsmksdispsgkyifrfyfngcfrkvviddrlpssktsr slymidrnhrnfmwpalvekaylklrggyefpgsnsgtdlwvltgwipeq vflhsdevtadqiwsdlfksfhsgdvlltigtgkltereqkelglvsehdyaild mkelkgrrqfliknpwagtdavypalfadpgpfpnspflspgtfwmdcem vlqnfenlylnwnpgifayqedihftwdlstgkgmagcfvknpqfsvyter ggvvwlllgrhlrtiesraseederfgfisiyvfkggkrvalsdgalhrgpyvd spntlmkldvpprslprvsqnftisafsdspvrishapnkyicvtkvqgswtp ttaggnaesaryslnpqfsivlsdptdisivlepsdqelathvklfwsggkriar vrsrdivadsgdyrrggslvekqdldpgeytivvstfapdqygsftlwvstnit cevtqlpseaagrravlsdigvllpgqdrmlaplttprltrvkliarsresrignr pvgpspllmtvelgqgpykeilatsedgdhsdsisgvrvedfdlqpgleerg gvwivleriggpggqvedhfevealgeerveigewiveda

\section{>A1CWF3.1 Aspergillus fischeri}

Mlsikrtllllgavlpavfgapvqetrraaqkipgkyivtfkpgtdaaties htlwatdlhkrnlerrdatsgeppigieknykikdfaayagsfddttieeirksa dvahveedqiwyidalttqkgapwglgsishkgqastdyiydtsagagtya yvvdtginvnhvefegraslaynaaggshvdsvghgthvagtiggktygva kktnllsvkvfqgessstsiildgfnwaandivskgrtrkaainmslgggysy afnnavenafdegvlsvvaagnentdasntspasapnaltvaainrsnarasf snygsvvdifapgqdilsawigsntatntisgtsmatphivglsvylmglesl sgpaavtsrikqlatngvvtnaqgspnklayngna

\section{>AAT85625.1 Aspergillus sp.}

Krnlerrdttsgetpvgieksykikdfaayagsfdaatieeirksadvahv eedqiwyidalttqrgapwglgsishkgqastdyiydtsagagtyayvvdtg invnhvefegraslaynaaggshvdsvghgthvagtiggktygvakktnlls vkvfqgessstsiildgfnwaandivskgrttkaainmslgggysyafnnav enafdegvlsvvaagnentdasntspasapnaltvaainrsnarasfsnygsv vdifapgqdilsawigsntatntisgtsmatphivglsvylmgleslsgpaavt srikqlatngvvtnarg

\section{>B8NLY9.1 Aspergillus flavus}

Mvilskvaavavglstvasalptgpshspharrgftinqitrqtarvgpkt asfpaiysralakyggtvpahlksavasghgtvvtspepndieyltpvniggtt lnldfdtgsadlwvfseelpkseqtghdvykpsgnaskiagaswdisygdg ssasgdvyqdtvtvggvtaqgqaveaaskisdqfvqdknndgllglafssin tvkpkpqttffdtvkdqldaplfavtlkyhapgsydfgfidkskftgelayad vddsqgfwqftadgysvgkgdaqkapitgiadtgttlvmlddeivdayykq vqgakndasaggyvfpcetelpeftvvigsynavipgkhinyaplqegsstc vggiqsnsglglsilgdvflksqyvvfdsqgprlgfaaqa 


\section{>P35211.1 Aspergillus flavus}

Mqsikrtllllgavlpavlagpifphrrapttipgkyivtfksdvdqaaid khtawatdihkrnlqrrdsseedlpigiernfkinkfaaysgsfdedtiaqirqs devaaveedqvwhlfdlttqsdapwglgsishkgqpstdyiydtnggegty ayvvdiginvdheefegraslayhaaggqhvdgvghgthvsgtiggktygv akkanllsvkvfvgessstsiildgfnwaandivskkrtgkaainmslgggy skafndavenafnegvlsivaagnentdasrtspasapdaftvaainvnntra yfsnygsvvdifapgqnilsawigsntatntisgtsmatphivglsiylmslev lsspkavsdrikelatrgvvsnvagspnllayngna

\section{>P28296.2 Aspergillus fumigatus}

Mlsikrtllllgavlpavfgapvqetrraaqkipgkyivtfkpgtdtaties htlwatdlhkrnlerrdttsgeppvgieksykikdfaayagsfddatieeirks advahveedqiwyldalttqkgapwglgsishkgqastdyiydtsagagty ayvvdsginvnhvefesraslaynaaggshvdsighgthvagtiggktygv akktnllsvkvfqgessstsiildgfnwavndivskgrtkkaainmslgggys yafnnavenafdegvlsvvaagnensdasntspasapnaltvaainksnara sfsnygsvvdifapgqdilsawigsttatntisgtsmatphivglsvylmglen lsgpaavtarikelatngvvtnvkgspnklayngna

\section{>P41748.2 Aspergillus fumigatus}

Mvvfskvtavvvglstivsavpvvqprkgftinqvarpvtnkktvnlp avyanaltkyggtvpdsvkaaassgsavttpeqydseyltpvkvggttlnldf dtgsadlwvfsselsasqssghaiykpsanaqklngytwkiqygdgssasg dvykdtvtvggvtaqsqaveaashissqfvqdkdndgllglafssintvsprp qttffdtvksqldsplfavtlkyhapgtydfgyidnskfqgeltytdvdssqgf wmftadgygvgngapnsnsisgiadtgttllllddsvvadyyrqvsgakns nqyggyvfpcstklpsfttviggynavvpgeyinyapvtdgsstcyggiqsn sglgfsifgdiflksqyvvfdsqgprlgfapqa

\section{>B0XRV0.1 Aspergillus fumigatus}

mgafrwlsiaaaastalaltpeqlitaprrseaipdpsgkvavfstsqysfe thkrtswwslldlktgqtkvltndssvseivwlsddsilyvnstnadipggvel wvtqassfakgykaaslpasfsglkaaktksgdirfvaygqsypngtaynee lataplssariydsiyvrhwdywlsttfnavfsgtlkkghgkngysldgelknl vspvknaespyppfggasdydlspdgkwvafkskapelpkanfttsyiylv phdasetarpingpdspgtpkgikgdssspvfspngdklayfqmrdetyes drrvlyvyslgskktipsvagdwdrspdsvkwtpdgktlivgsedlgrtrlfsl panakddykpknftdggsvsayyflpdssllvtgsalwtnwnvytakpekg vikkiasaneidpelkglgpsdisefyfqgnftdihawviypenfdkskkyp liffihggpqgnwadgwstrwnpkawadqgyvvvapnptgstgfgqaltd aiqnnwggapyddlvkcweyvhenldyvdtdhgvaagasyggfminwi qgsplgrkfkalvshdgtfvadakvsteelwfmqrefngtfwdardnyrrw dpsaperilqfatpmlvihsdkdyrlpvaeglslfnvlqergvpsrflnfpden hwvvnpenslvwhqqalgwinkysgveksnpnavsledtvvpvvnyn

\section{>P0C959.1 Aspergillus fumigatus}

Mgafrwlsiaaaastalaltpeqlitaprrseaipdpsgkvavfstsqysf ethkrtswwslldlktgqtkvltndssvseivwlsddsilyvnstnadipggv elwvtqassfakgykaaslpasfsglktaktksgdirfvaygqsypngtayne elataplssariydsiyvrhwdywlsttfnavfsgtlkkghgkngysldgelk nlvspvknaespyppfggasdydlspdgkwvafkskapelpkanfttsyiy lvphdasetarpingpdspgtpkgikgdssspvfspngdklayfqmrdety esdrrvlyvyslgskktipsvagdwdrspdsvkwtpdgktlivgsedlgrtrl fslpanakddykpknftdggsasayyflpdssllvtgsalwtnwnvytakpe kgvikkiasaneidpelkglgpsdisefyfqgnftdihawviypenfdkskkypl iffihggpqgnwadgwstrwnpkawadqgyvvvapnptgstgfgqaltdaiq nnwggapyddlvkcweyvhenldyvdtdhgvaagasyggfminwiqgspl grkfkalvshdgtfvadakvsteelwfmqrefngtfwdardnyrrwdpsaperi lqfatpmlvihsdkdyrlpvaeglslfnvlqergvpsrflnfpdenhwvvnpens lvwhqqalgwinkysgveksnpnavsledtvvpvvnyn

\section{$>$ O42630.1 Aspergillus fumigatus}

Mkstslltasvllgsasaavhklklnkvpldeqlythnidahvralgqky mgirpnvhqelleenslndmsrhdvlvdnflnaqyfseislgtppqkfkvvl dtgssnlwvpgsdcssiacflhnkydssasstykangtefaikygsgelsgfv sqdtlqigdlkvvkqdfaeatnepglafafgrfdgilglgydtisvnkivppfy nmldqglldepvfafylgdtnkegdnseasfggvdknhytgeltkiplrrka ywevdfdaialgdnvaelentgiildtgtslialpstladllnkeigakkgftgq ysiecdkrdslpdltftlaghnftigpydytlevqgscissfmgmdfpepvgp lailgdaflrkwysvydlgnnavglakak

\section{>A2R3L3.1 Aspergillus niger}

Mvvfsktaalvlglssavsaapaptrkgftinqiarpanktrtinlpgmy arslakfggtvpqsvkeaaskgsavttpqnndeeyltpvtvgkstlhldfdtgs adlwvfsdelpsseqtghdlytpsssatklsgytwdisygdgssasgdvyrdt vtvggvttnkqaveaaskissefvqntandgllglafssintvqpkaqttffdtv ksqldsplfavqlkhdapgvydfgyiddskytgsitytdadssqgywgfstd gysigdgsssssgfsaiadtgttlillddeivsayyeqvsgaqeseeaggyvfs cstnppdftvvigdykavvpgkyinyapistgsstcfggiqsnsglglsilgd vflksqyvvfnsegpklgfaaqa

\section{>XP_001391470.1 Aspergillus niger}

Mkgilglsllplltaaspvfvdsihneaapilsatnakevpdsyivvfkk hvtselasahhswvqdihdsqsertelkkrslfglgdevylglkntfdiagsli gysghfhedvieqvrrhpdvdyierdsevhtmegateknapwglarishrd sltfgnfnkylyaseggegvdaytidtginvdhvdfegratwgktiptndedl dgnghgthcsgtmagkkygvakkanlyavkvlrssgsgtmsdvvsgvey avqahikkakdakngkvkgfkgsvanmslgggksktledavnagveagl hfavaagndnadacnyspaaaekaitvgastladerayfsnygectdifapgl nilstwigsnyatniisgtsmasphiagllayfvslqppsdsafaveeltpaklk kdiiaiategaltdipsntpnllawngggsenytdivgsggykvssaknried rieglvhkaeellteelgaiyseiqdavva

\section{>P55325.1 Aspergillus niger}

Mvvfsktaalvlglstavsaapaptrkgftinqiarpanktrtvnlpglya rslakfggtvpqsvkeaaskgsavttpqnndeeyltpvtvgkstlhldfdtgsa dlwgfsdelpsseqtghdlytpsssatklsgyswdisygdgssasgdvyrdt vtvggvttnkqaveaaskissefvqdtandgllglafssintvqpkaqttffdtv ksqldsplfavqlkhdapgvydfgyiddskytgsitytdadssqgywgfstd gysigdgsssssgfsaiadtgttlillddeivsahyeqvsgaqesyeaggyvfs 
cstdlpdftvvigdykavvpgkyinyapvstgsstcyggiqsnsglglsilgd vflksqyvvfnsegpklgfaaqa

\section{>P24665.1 Aspergillus niger}

mkfstiltgslfataalaapltekrrarkearaagkrhsnppyipgsdkeil klngttneeyssnwagavligdgytkvtgeftvpsvsagssgssgygggyg ywknkrqseeycasawvgidgdtcetailqtgvdfcyedgqtsydawye wypdyaydfsditisegdsikvtveatskssgsatvenlttgqsvthtfsgnve gdlcetnaewivedfesgdslvafadfgsvtftnaeatsggstvgpsdatvm dieqdgsvltetsvsgdsvtvtyv>CAK42925.1Aspergillusniger mdlfhsagfasdvtelmkqqhvpglaiaiihndqiasagyghasldpeipct adtlfdiassaksltaaavgllvddndmfpdiqydavmstllpedfvmsgkg ytegvtvedilshrsgmpghddsymsvraakpdnarsitrnlrnlpvaapir skyiycnmmytvathlvevksgqdfgtfledrffkpldmasttlqpssarsk gfgsrmatgytwkradstyrglespdcpegqgagsiissvndfikfvkafm nredpinknvyegltrlrtfvnpnpgrrkrysspvayaagldvyfykghmv vghngafsgfasrffflpdfsfgavimgnsdgangiattlvqklidnvlgvtde kpqdskskdtrsveirgpkpqaeakdanpmsknqekkekkkqekksqak ksqgiqkgqvneqkpnrntpqpptlplsayagnywnpgyhnlqvqirdda lfidatdrsmgftlkfehvsddrkfnahltdwldgsddivkaefvieddqvtrl glqleemlqemiwfekkdgvrsgaarvlvp

\section{>A2Q7V4.1 Aspergillus niger}

Mhglrlvcsigtlplvilaypaaslhttsaavdldslrltsnseyvnsvhvd tnrsvavsaeehytdtaarlvqnivpgasfrliddhfvgdngvahvyfrqtlh ididnadfnvnigkdglvlsfghsfftgalpsshldntnvlspeaalrgardaiq lpltidnvsteaaegrneyifreavgavsdpkaklvylvkpegtlaltwrietd myehwlltyidaetttvhgvvdyvadatyqvypwgtndpaeghrtivtdp wdlsasaytwisdgrdnytttrgnnaiahwnptgggsylynlrpsdpnlnfq wpyspnmspprsyinasivqlfytanayhdllytlgftesagnfqwnnsah ggrdkdyvilnaqdgsgfsnanfatppdgipgrmrmyiwiestpsrdgs fdagiviheythgvsnrltggshnagclsalesggmgegwgdfmatairi kpndtrttsytmgawadndkcgvrdypystsftenplnytsvntmngv haigtvwatmlyevlwnlidkygkndgsrpvfrngvptdgkylmmkl vvdgmalqpcnpnfvqardaildadivltggknrceiwrgfakrglgqg aahsslnwmrrgstllptgc

\section{>CAK44878.1 Aspergillus niger}

Mflispavtvaaalllingagatqsersraaahfskrhptyraatraqssnt sdyrffnnrtkphlveslpdvhfdvgemysgsipiddsnngsrslfyifqpki gepsddltiylnggpgcsseqgffqengrftwqpgtyapvineyswvnltn mlwvdqpvgtgfsvgnvtatneeeiaadfldffekfedlygiknfrifmtge syagryvpyissamldkndttrfnlsgallydacigqwdyiqaelpaypfvk qhaslfnfnqsymnelettyeecgykayfdeyfafppsgiqppkymnyse cdiynmiyyeaynpnpcfnpyrvidecpllwdvlgwptdlayepapttyf nridvkkalhapmdvewelcsydlvfaggdadpgpeqqgddspnptegv lprvieatnrvliangdwdyliitngtllaiqnmtwngqlgfqsapatpidiq mpdlqwveifeaqegyggldgpqgvmgvqhyerglmwaetyqsghkq aqdqgrvsyrhlqwllgqveil

\section{>Q00070 Aspergillus niger}

Mksaslltasvllgcasaevhklklnkvpleeqlythnidahvralgqky mgirpsihkelveenpindmsrhdvlvdnflnaqyfseielgtppqkfkvvl dtgssnlwvpssecssiacylhnkydssasstyhkngsefaikygsgslsgfv sqdtlkigdlkvkgqdfaeatnepglafafgrfdgilglgydtisvnkivppfy nmldqglldepvfafylgdtnkegdesvatfggvdkdhytgelikiplrrka yweveldaialgddvaementgvildtgtslialpadlaeminaqigakkg wtgqytvdcdkrsslpdvtftlaghnftissydytlevqgscvsafmgmdfp epvgplailgdaflrkwysvydlgnsavglakak

\section{>P55325.1 Aspergillus niger}

Mvvfsktaalvlglstavsaapaptrkgftinqiarpanktrtvnlpglya rslakfggtvpqsvkeaaskgsavttpqnndeeyltpvtvgkstlhldfdtgsa dlwgfsdelpsseqtghdlytpsssatklsgyswdisygdgssasgdvyrdt vtvggvttnkqaveaaskissefvqdtandgllglafssintvqpkaqttfdtv ksqldsplfavqlkhdapgvydfgyiddskytgsitytdadssqgywgfstd gysigdgsssssgfsaiadtgttlillddeivsahyeqvsgaqesyeaggyvfs cstdlpdftvvigdykavvpgkyinyapvstgsstcyggiqsnsglglsilgd vflksqyvvfnsegpklgfaaqa

\section{>A2R2G1.1 Aspergillus niger}

Mklsialalgatastgvlaavvpqqeplitpqdppthhhqekflielapy qtrwvteeekwdlkldgvnfiditeerntgfyptlhagsyvhypptmkhae kvvpllrglskdnmeqnlnkftsfhtryyrsstgiesakwlysrvsdvieqsg aaeygatveqfahswgqfsiiaripgqtnktvvlgahqdsinlflpsilaapga dddgsgtvtilealrgllqsdaivrgnasntiefhwysaeeggmlgsqaifsq ykrdkrdikamlqqdmtgytqgaldagrqeaigimvdyvdegltqflkdvt teycgigyietrcgyacsdhtsaskygypaamatesemensnkrihttddsir ylsfdhmleharltlgfayelafaqf

\section{>Q8NK92.2 Aspergillus oryzae}

Mrplshlsffnglllglsalsaatsvvherreatssnwvkrarvnpsdkh vvrigltqssleeahdllmdvsnpsspnyarfysadevaakfapstetvnevq nwltekginasrvaqtqnhgwlvfhatskeienlfdttyyeyhnrktgkkaia ceqyhvpasvqkhidyvhpgvnlnpssgkpssirrraaaskktklpargpr piqqhdvkglnvtncdqlitpeciralykipsaraaphpnnslgifeegdyya qedldlffktfakdipqgthpipafidgaeapvpvtkaggesdldfelaypiv hpqsitlyqtddanwasnttgflntfldaldgsyctycaygecgndpsldpvy pddagydgqlmcgvfkptnvisvsygeqendlpanyqqrqcmeflklglq gvsvlfasgdngvagppgdgnsvngclnngtvfspafpnscpyitnvgatk vypgytvsqpesavydpdglysyasgggfsniypipdyqaeavatyfkdh nppypyyegaenlgkngglynrlgrgypdvaangdniavfnggefgssgg tsastpifasiinriiderlavgkgpvgfinpvlyknpsvlnditngtnpgcgtd gfstapgwdpatglgtpnypkmlklwldlp

\section{>Q2U319 Aspergillus oryzae}

Mkpillavplllsytaaeihrvplekellvfgsddddtrtssqryigsnthq kalqdhgpdilghdipvknhrntqyfstirigtppqkfkvvldtgsanlwvps skcktisckkhkkyksalsdtyhnngsefeiyygsggmtghvsediftigdl kvqeqlfgeatkvsgfsnvkadgilglgfasisvnsipppfynmldqnllde pvfafylsdtykgrtseitfggvdeqhysgeivkiplrrkaywevefsglffgd hfadvedtgailntgssliglpsglfetvnkeigatrdyqgryildcdkrsfmps ltfvlgeynftidpkdyslqeqnfcmsalvpmdfpgptgplvvlgdaflrrw ysvydfgngaiglaqakrke 
>Q2UDE1 Aspergillus oryzae

Mrfllsflitlsiacgvlslpsgsksqlkgrsfkvervrrgnepvhgptalrr ayekfgivptdlgidlddfepimtkhavvekkdvtepdqkgavsassvlgd aafvspviiggqkvvlnfdtgsadfwvmntelpaeaqkgrtvynpsnsstfk kmegatfnisygdasyayggvgtdtvnvggaivkdqaigipdtvssafiedttsn glvglgfsslntvkpkqqktffdniadslqepvmtaslkangvgeyefgildhdk yqgdianvsvdsskgfwqfelakfavadgdiqtikenptaiadtgtslmllsqev vdayyakiegaiyassasgyiypcnaslpsisvaigsnhlatvpgnlinfsevgin kttggkvcfggvqsnqgssmqilgdvflkaffvvfdlrgpslgvaspk

\section{>AAC60533.1 Aspergillus oryzae}

Mqsikrtllllgailpavlgapvqetrraaeklpgkyivtfkpgideakiq ehttwatnihqrslerrgatggdlpvgiernykinkfaayagsfddatieeirk nedvayveedqiyyldglttqksapwglgsishkgqqstdyiydtsagegty ayvvdsgvnvdheefegraskaynaaggqhvdsighgthvsgtiagktygi akkasilsvkvfqgessstsvildgfnwaandivskkrtskaainmslgggy skafndavenafeqgvlsvvaagnensdagqtspasapdaitvaaiqksnnr asfsnfgkvvdvfapgqdilsawigsssatntisgtsmatphivglslylaale nldgpaavtkrikelatkdvvkdvkgspnllayngna

\section{>Q2UUW3 Aspergillus oryzae}

Mrgasllpvalaalscvdalslhrrdtpatvelpierrqhagglqkrdstln lplinyydsfyilnltlgtpaqqfavaldtgssdlwvnvanssycssrtnpckp fglydpdasstyknlgvefnatygdgtnaygyyatdelglgdvnvddmqfg vaesttitqgivgvaydtltneashegktyvnlpqalvnsgaikspayslwln dpqasrgsilfggvnkakykgelqtipivrtlrgysylavtltgvsveqgkese dyssrlpivvlldsgtsltylpdslvdelyktfnatfleddglayvdcelmkkdytv nfdfsgatiavgiselvlkavaedfplgtcafgvvpsgdsqdamyilgdtflrsay vvydlgnneislantnfspgdddileigtgtsavpgatpvespvtsatvasatdivh tvmvggtkatatdsnsgaaetssssgiaalptsntrhllsglagaglllal

\section{$>$ Q8NKB2 Aspergillus oryzae}

Mkstlvtasvllgcasaevhklklnkvpvseqfnlhnidthvqalgqky mgirpnikqdllnenpindmgrhdvlvdnflnaqyfseieigtppqkfkvvl dtgssnlwvpssecgsiacylhnkydssssstyqkngsefaikygsgslsgfv sqdtlkigdlkvkdqlfaeatsepglafafgrfdgilglgfdtisvnkipppfys mldqglldepvfafylgdtnkegddsvatfggvdkdhytgelvkiplrrkay wevdldaialgdsvaeldntgvildtgtslialpttlaelinkeigakkgftgqys vdcdkrdslpdltftlsgynftigpydytlevqgscisafmgmdfpepvgpl ailgdaflrkwysvydlgngavglakak

>Q8NKB6.1 Aspergillus oryzae

Mqkswlvllvaclglqgttaltlhrrdlpavvsldikrnnavdpvardrm rrkrdktveqnldneetlyfenitlgtpkqslrlvldtgssdlwcnaanstlcssr dqpcnasgsydpsssssyaytssdfnisyadgtgaagdyvtdtihiggatvk dfqfgvgyssssaegvlgigyttnevqvgrlgksayanlpqamvkngliqs nayslwlndlgadtgsilfggvntekyhgelqtlpiqtvngvysefiialtgvsl ssasshhnysssdalpaavlldsgssltylpnsivqdiyddlgvtyesssgvgyv pcslaqqninvtytfsspiitvgidelvldagdlrfrngaracifgivpagdstavlg dtflrsayvvydlsnneislantkfnstkdnileigtgddsvpgatqusnpvtsvva dgsgariggptgeiftdipsatssggaaapagptdvpkhlvlgaaaigyvlaf
>AAB20819.1 Aspergillus oryzae

Mqsikrtllllgailpavlgapvqetrraaeklpgkyivtfkpgideakiq ehttwatnihqrslerrgatggdlpvgiernykinkfaayagsfddatieeirk nedvayveedqiyyldglttqksapwglgsishkgqqstdyiydtsagegty ayvvdsgvnvdheefegraskaynaaggqhvdsighgthvsgtiagktygi akkasilsvkvfqgessstsvildgfnwaandivskkrtskaainmslgggy skafndavenafeqgvlsvvaagnensdagqtspasapdaitvaaiqksnnr asfsnfgkvvdvfapgqdilsawigsssatntisgtsmatphivglslylaale nldgpaavtkrikelatkdvvkdvkgspnllayngna

\section{>Q06902.2 Aspergillus oryzae}

Mvilskvaavavglstvasalptgpshspharrgftinqitrqtarvgpkt asfpaiysralakyggtvpahlksavasghgtvvtspepndieyltpvniggtt lnldfdtgsadlwvfseelpkseqtghdvykpsgnaskiagaswdisygdg ssasgdvyqdtvtvggvtaqgqaveaaskisdqfvqdknndgllglafssin tvkpkpqttffdtvkdqldaplfavtlkyhapgsydfgfidkskftgelayad vddsqgfwqftadgysvgkgdaqkapitgiadtgttlvmlddeivdayykq vqgakndasaggyvfpcetelpeftvvigsynavipgkhinyaplqegsstc vggiqsnsglglsilgdvflksqyvvfdsqgprlgfaaqa

\section{>BAA00951.1 Aspergillus oryzae}

mqsikrtllllgailpavlgapvqetrraaeklpgkyivtfkpgideak iqehttwatnihqrslerrgatggdlpvgiernykinkfaayagsfddatie eirknedvayveedqiyyldglttqksapwglgsishkgqqstdyiydts agegtyayvvdsgvnvdheefegraskaynaaggqhvdsighgthvsg tiagktygiakkasilsvkvfqgessstsvildgfnwaandivskkrtska ainmslgggyskafndavenafeqgvlsvvaagnensdagqtspasap daitvaaiqksnnrasfsnfgkvvdvfapgqdilsawigsssatntisgts matphivglslylaalenldgpaavtkrikelatkdvvkdvkgspnllay ngna>CAA38527.2Aspergillusoryzaemqsikrtllllgailpa vlgapvqetrraaeklpgkyivtfkpgideakiqehttwatnihqrslerrg atggdlpvgiernykinkfaayagsfddatieeirknedvayveedqiyy ldglttqksapwglgsishkgqqstdyiydtsagegtyayvvdsgvnvd heefegraskaynaaggqhvdsighgthvsgtiagktygiakkasilsvk vfqgessstslildgfnwaandivskkrtskaainmslgggyskafndav enafeqgvlsvvaagnensdagqtspasapdaitvaaiqksnnrasfsnf gkvvdvfapgqdilsawigsssatntisgtsmatphivglslylaalenld gpaavtkrikelatkdvvkdvkgspnllayngna

\section{>Q9Y8E3.2 Aspergillus oryzae}

Mgalrwlsiaatastalalnpeglisaprrseaipnpsgdvavfsqsqysf kthkttsqwnvldlksgdiklltndsdvseivwlgsddstvlyvngtnadipg gvelwvsdisdfangykaaslpasfsgfkvvttdsgdvryvayaeswangt ayneelvakplssariydsiyvrhwdyylttrfnavfsgtlkksegkgkaty kadgdlknlvspvknaespyppfggasdydlspdgkwvafkskahdi pranyttayiflvphdgsktavpingpdspgtpegvkgdagspvfspds kkiaywqmadesyeadhrtlyvytvgseetipslaadwdrsldsvkwa dddnliigvedagrsrlfsipadagddykpknftdggvvsayyqlpdsty 
lvtstaiwtswnvyiaspekgviktlatankidpelkglgpeivdefyyeg nwtkiqafviypenfdksksypllyyihggpqsswldswstrwnpkvf adqgyvvvapnptgssgfgdalqdaiqnqwggypyedlvkgweyvn enfdfidtdngvaagasyggfminwiqgsdlgrkfkalvshdgtfvada kvsteelwfmqhefngtfwdnrenyrrwdpsaperilkfstpmliihsdl dyrlpvseglslfnilqergvpsrflnfpdenhwvqnkenslvwhqqvl gwlnkysgveesnedavsldntvipvvdynp

\section{>P46076.2 Aspergillus oryzae}

Mrvttlstalfalastavsaptagssspglevkltqidntrvkavvkntgse evsfvhlnffkdagpvkkvsiyrgqdevqfegikrrlrssgitkeavtslgage tledefdiastsdlasggpvsirshgfvpivvdgkitgyipyksndltvnvdgg kaakvtkalsqltrrtevtdckgdaesslttalsnaaklanqaaeaaesgdeskf eeyfkttdqqtrttvaerlravakeagstsggsttyhcndpygycepnvlaytl pskneiancdiyyselpplaqkchaqdqatttlhefthapgvyqpgtedlgy gydaatqlsaqdalnnadsyalyanaielkc

\section{>Q2TWA0 Aspergillus oryzae}

Mkliqlssvlhftalssaltlplnrrspceeetshnapllatlggsvfdv dvtigadnqtfkllvdtgssdtyimqdgftcinatdnqiiapedckygpet yhvsssyeqvpdqnfgieygaglasgvmayetitiadvtvrtklafadrs hpmgdgvnngllglgypsltsahpgtftpndtyfynravynpvvnemy eqglieepyfsialahtardstgafggyislgelppveltsefttvpveimen ipinitsdkrqisywafttpavkygpaeeeedalvvdhtpfqlfidtgnefs ilptavvdpvntrfeppavyndelkayivdcgakppvfgvvvgnqtfyh apedliydtgngycvstlvpsekngrpvlvinilgvpffknlvavfdfgkd emrfarvsngyfl

\section{>Q8NKB5 Aspergillus oryzae}

mmrgasllpvalaalscvdalslhrrdtpatvelpierrqhagglqkr dstlnlplinyydsfyilnltlgtpaqqfavaldtgssdlwvnvanssycss rtnpckpfglydpdasstyknlgvefnatygdgtnaygyyatdelglgdv nvddmqfgvaesttitqgivgvaydtltneashegktyvnlpqalvnsga ikspayslwlndpqasrgsilfggvnkakykgelqtipivrtlrgysylavt ltgvsveqgkesedyssrlpivvlldsgtsltylpdslvdelyktfnatfled dglayvdcelmkkdytvnfdfsgatiavgiselvlkavaedfplgtcafg vvpsgdsqdamyilgdtflrsayvvydlgnneislantnfspgdddilei gtgtsavpgatpvespvtsatvasatdivhtvmvggtkatatdsnsgaaet ssssgiaalptsntrhllsglagaglllal>Q12567.1Aspergillusph oenicismvvfsktaalvlglstavsaapaptrkgftinqiarpanktrtvnl pglyarslakfggtvpqsvkeaaskgsavttpqnndeeyltpvtvgkstlh ldfdtgsadlwvfsdelpsseqtghdlytpsssatklsgyswdisygdgss asgdvyrdtvtvggvttnkqaveaaskissefvqdtandgllglafssint vqpkaqttffdtvksqldsplfavqlkhdapgvydfgyiddskytgsityt dadssqgywgfstdgysigdgsssssgfsaiadtgttlillddeivsayyeq vsgaqesyeaggyvfscstdlpdftvvigdykavvpgkyinyapvstgs stcyggiqsnsglglsilgdvflksqyvvfnsegpklgfaaqa

\section{>A1DDK1.1 Aspergillus fischeri}

mvvfskvtavvvglstivsavpvvqprkgftinqvarpvtnkktvnlpa vyanaltkyggtvpdsvkaaassgsavttpeqydseyltpvkvggttlnldfd tgsadlwvfsselsasessghaiykpsanaqklngytwkiqygdgsgasgd vykdtvtvggvtaqsqaveaasqissqfvqdkdndgllglafsslntvsprpq ttffdtvksqldsplfavtlkyhapgtydfgyidnskfqgkltyadvdnsqgf wmftadgygvgdgapnsnhisgiadtgttllllddsvvadyyhqvsgakns neyggyvfpcstklpsfttviggynavvpgeyinyapvtdgsstcfggiqsn sglgfsifgdvflksqyvvfdsqgprlgfapqa 